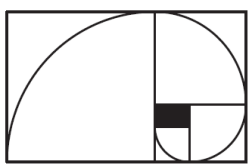

https://doi.org/10.21711/217504322019/em341

\title{
Malliavin calculus and normal approximations
}

\section{David Nualart}

\begin{abstract}
The goal of these notes is to introduce the basic elements of Malliavin calculus and to discuss its applications to quantitative normal approximations. The basic differential operators, the derivative and the divergence, are first introduced in the framework of the finite-dimensional Euclidean space equipped with the standard normal distribution. Later, we study these operators on the Wiener space and their relation with Wiener chaos expansions and the Ornstein-Uhlenbeck semigroup. Chapter 2 is devoted to the application of Malliavin calculus, combined with Stein's method, to derive estimations of the total variation distance in normal approximations. These estimates are applied, in Chapters 3 and 4, to the Breuer-Major theorem for stationary sequences and to spatial averaging of stochastic partial differential equations, respectively.
\end{abstract}

2010 Mathematics Subject Classification: 60H07, 60F05, $60 \mathrm{H} 15$.

Keywords: Malliavin calculus, Wiener chaos expansions, Ornstein-Uhlenbeck semigroup, Stein's method for normal approximations, total variation distance, central limit theorem, Breuer-Major theorem, fractional Brownian motion, fourth moment theorem, stochastic heat equation. 



\section{Contents}

1 Malliavin calculus 5

1.1 Finite-dimensional case . . . . . . . . . . . . . 5

1.2 Malliavin calculus on the Wiener space . . . . . . . . 6

1.2.1 Brownian motion and Wiener space . . . . . . . 6

1.2 .2 Wiener integral . . . . . . . . . . . . . 8

1.2.3 The derivative operator . . . . . . . . . . . . . 8

1.2 .4 Sobolev spaces . . . . . . . . . . . . . . . . . 11

1.2 .5 Chain rule . . . . . . . . . . . . . . 12

1.2.6 Domain of the divergence in $L^{2}(\Omega) \ldots \ldots \ldots . \ldots 12$

1.2 .7 Iterated derivatives . . . . . . . . . . . . . . 13

1.2 .8 Continuity of the divergence . . . . . . . . . . 13

1.2.9 The divergence as a stochastic integral . . . . . . . . 14

1.2 .10 Clark-Ocone formula . . . . . . . . . . . . 15

1.2.11 Isonormal Gaussian processes . . . . . . . . . . . 17

1.3 Multiple stochastic integrals. Wiener chaos . . . . . . . 17

1.3.1 Hermite polynomials . . . . . . . . . . . . . 18

1.3.2 Multiple Stochastic Integrals . . . . . . . . . . 20

1.3.3 Wiener chaos expansion . . . . . . . . . . . . . 22

1.3.4 Derivative operator on the Wiener chaos . . . . . . 22

1.3.5 Divergence on the Wiener chaos . . . . . . . . . . 24

1.4 Criterion of differentiability . . . . . . . . . . 25

1.5 Ornstein-Uhlenbeck semigroup . . . . . . . . . . . 26

1.5.1 Mehler's formula . . . . . . . . . . . . . . 26

1.5.2 Hypercontractivity . . . . . . . . . . . . . 27

1.5.3 Generator of the Ornstein-Uhlenbeck semigroup . . 28

1.5.4 Second integral representation . . . . . . . . . . 29

1.6 Existence and regularity of densities . . . . . . . . . . 30

2 Malliavin-Stein's approach $\quad 32$

2.1 Stein's method for normal approximations . . . . . . . . . . 32

2.1.1 Total variation and convergence in law . . . . . . . 34

2.2 Stein meets Malliavin . . . . . . . . . . . . . . . 35 
2.2.1 Normal approximation on a fixed Wiener chaos . . . 37

2.2.2 Fourth Moment theorem . . . . . . . . . . . . 38

2.2.3 Multivariate Gaussian approximation . . . . . . . . 40

2.2.4 Chaotic Central Limit Theorem . . . . . . . . . . . . 40

3 Central limit theorems for stationary sequences 41

3.1 Breuer-Major theorem . . . . . . . . . . . . . . . 41

3.1 .1 Convergence in law in $C([0, T]) \ldots \ldots \ldots$

3.1.2 Functional version of the Breuer-Major theorem . . 45

3.1.3 Rate of convergence . . . . . . . . . . . . . . 49

3.1.4 Optimal rate of convergence . . . . . . . . . . 53

3.2 Fractional Brownian motion . . . . . . . . . . . . . 55

3.2 .1 Fractional noise . . . . . . . . . . . . . 56

3.2.2 Asymptotic behavior of the the $q$-variation of the $\mathrm{fBm} 57$

4 Spatial averaging of SPDEs $\quad 58$

4.1 Stochastic heat equation . . . . . . . . . . . . . 58

4.1.1 Stochastic integration . . . . . . . . . . 58

4.1 .2 Mild solution . . . . . . . . . . . . . . . 59

4.1.3 Malliavin differentiability of the solution . . . . . . 59

4.2 Space averages . . . . . . . . . . . . . 63

4.2.1 Functional Central Limit Theorem . . . . . . . . . 66

4.2 .2 Spatial colored noise . . . . . . . . . . . . . 68

4.2 .3 Stochastic wave equation . . . . . . . . . . 70

$\begin{array}{lr}\text { Bibliography } & 72\end{array}$ 


\section{Chapter 1}

\section{Malliavin calculus}

Motivated by a probabilistic proof of Hörmander's hypoellipticity theorem (see [13]), Malliavin introduced in the 70's a calculus of variations with respect to the trajectories of the Brownian motion. This calculus was further developed by Bismut, Kusuoka, Stroock, and Watanabe [3, 12, 28, 30], among others. The Malliavin calculus is a differential calculus on a Gaussian probability space. Its main application has been to establish the existence and smoothness of densities of functionals of Gaussian processes. In combination with Stein's method, the Malliavin calculus has been recently used to derive quantitative results on normal approximations. Basic references for Malliavin calculus and its applications to normal approximations are $[16,19,20]$.

\section{$1.1 \quad$ Finite-dimensional case}

Consider first the finite-dimensional case. That is, the probability space $(\Omega, \mathcal{F}, P)$ is such that $\Omega=\mathbb{R}^{n}, \mathcal{F}=\mathcal{B}\left(\mathbb{R}^{n}\right)$ is the Borel $\sigma$-field of $\mathbb{R}^{n}$, and $P$ is the standard Gaussian probability with density $p(x)=(2 \pi)^{-n / 2} e^{-|x|^{2} / 2}$. In this framework we consider two differential operators. The first one is the derivative operator, which is simply the gradient of a differentiable function $F: \mathbb{R}^{n} \rightarrow \mathbb{R}:$

$$
D F=\left(\frac{\partial F}{\partial x_{1}}, \ldots, \frac{\partial F}{\partial x_{n}}\right) .
$$

The second differential operator is the divergence operator and is defined on differentiable vector-valued functions $u: \mathbb{R}^{n} \rightarrow \mathbb{R}^{n}$ as follows:

$$
\delta(u)=\sum_{i=1}^{n}\left(u_{i} x_{i}-\frac{\partial u_{i}}{\partial x_{i}}\right)=\langle u, x\rangle-\operatorname{div} u .
$$


We denote by $C_{p}^{k}\left(\mathbb{R}^{n} ; \mathbb{R}^{m}\right)$ is the space of functions $f: \mathbb{R}^{n} \rightarrow \mathbb{R}^{m}$ which are $k$ times continuously differentiable and their partial derivatives up to order $k$ are bounded by $C\left(1+|x|^{N}\right)$ for any $x \in \mathbb{R}^{n}$ and for some real number $N \geq 0$. That means $f$ and its partial derivatives up to the order $k$ have polynomial growth.

It turns out that $\delta$ is the adjoint of the derivative operator with respect to the Gaussian measure $P$. This is the contents of the next proposition.

Proposition 1.1.1. The operator $\delta$ is the adjoint of $D$; that is,

$$
\mathbb{E}(\langle u, D F\rangle)=\mathbb{E}(F \delta(u))
$$

if $F \in C_{p}^{1}\left(\mathbb{R}^{n}\right)$ and $u \in C_{p}^{1}\left(\mathbb{R}^{n} ; \mathbb{R}^{n}\right)$.

Proof. Integrating by parts, and using $\partial p / \partial x_{i}=-x_{i} p(x)$, we obtain

$$
\begin{aligned}
\int_{\mathbb{R}^{n}}\langle D F, u\rangle p(x) d x & =\sum_{i=1}^{n} \int_{\mathbb{R}^{n}} \frac{\partial F}{\partial x_{i}} u_{i} p(x) d x \\
& =\sum_{i=1}^{n}\left(-\int_{\mathbb{R}^{n}} F \frac{\partial u_{i}}{\partial x_{i}} p(x) d x+\int_{\mathbb{R}^{n}} F u_{i} x_{i} p(x) d x\right) \\
& =\int_{\mathbb{R}^{n}} F \delta(u) p(x) d x .
\end{aligned}
$$

This completes the proof.

\subsection{Malliavin calculus on the Wiener space}

\subsubsection{Brownian motion and Wiener space}

The Wiener space is a probability space $(\Omega, \mathcal{F}, P)$ where

- $\Omega=C([0, T])$ is the space of continuous functions $\omega:[0, T] \rightarrow \mathbb{R}$.

- $\mathcal{F}$ is the Borel $\sigma$-field $\mathcal{B}(\Omega)$ for the topology of the uniform convergence. One can easily show that $\mathcal{F}$ coincides with the $\sigma$-field generated by the collection of cylinder sets

$$
C=\left\{\omega \in \Omega: \omega\left(t_{1}\right) \in A_{1}, \ldots, \omega\left(t_{k}\right) \in A_{k}\right\},
$$

for any integer $k \geq 1$, Borel sets $A_{1}, \ldots, A_{k}$ in $\mathbb{R}$, and $0 \leq t_{1}<\cdots<$ $t_{k} \leq T$.

- $P$ is the Wiener measure. That is, $P$ is defined on a cylinder set of the form (1.1) by

$$
P(C)=\int_{A_{1} \times \cdots \times A_{k}} p_{t_{1}}\left(x_{1}\right) p_{t_{2}-t_{1}}\left(x_{2}-x_{1}\right) \cdots p_{t_{k}-t_{k-1}}\left(x_{k}-x_{k-1}\right) d x_{1} \cdots d x_{k},
$$


where $p_{t}(x)$ denotes the Gaussian density $p_{t}(x)=(2 \pi t)^{-1 / 2} e^{-x^{2} /(2 t)}$, $x \in \mathbb{R}, t>0$.

The canonical process, defined by $B_{t}(\omega)=\omega(t)$, is a Brownian motion. That is, $B=\left(B_{t}\right)_{t \in[0 . T]}$ is a stochastic process satisfying the following properties:

(i) $B_{0}=0$.

(ii) For all $0 \leq t_{1}<\cdots<t_{n} \leq T$ the increments $B_{t_{n}}-B_{t_{n-1}}, \ldots, B_{t_{2}}-$ $B_{t_{1}}$ are independent random variables.

(iii) If $0 \leq s<t \leq T$, the increment $B_{t}-B_{s}$ is a Gaussian random variable with mean zero and variance $t-s$.

Properties (i), (ii), and (iii) are equivalent to saying that $B$ is a Gaussian process with mean zero and covariance function

$$
\Gamma(s, t)=\min (s, t) .
$$

The existence of Brownian motion can be proved in the following way: The function $\Gamma(s, t)=\min (s, t)$ is symmetric and nonnegative definite because it can be written as

$$
\min (s, t)=\int_{0}^{T} \mathbf{1}_{[0, s]}(r) \mathbf{1}_{[0, t]}(r) d r, \quad s, t \in[0, T] .
$$

Then, for any integer $n \geq 1$ and real numbers $a_{1}, \ldots, a_{n}$,

$$
\begin{aligned}
\sum_{i, j=1}^{n} a_{i} a_{j} \min \left(t_{i}, t_{j}\right) & =\sum_{i, j=1}^{n} a_{i} a_{j} \int_{0}^{T} \mathbf{1}_{\left[0, t_{i}\right]}(r) \mathbf{1}_{\left[0, t_{j}\right]}(r) d r \\
& =\int_{0}^{T}\left(\sum_{i=1}^{n} a_{i} \mathbf{1}_{\left[0, t_{i}\right]}(r)\right)^{2} d r \geq 0
\end{aligned}
$$

Therefore, by Kolmogorov's extension theorem, there exists a Gaussian process with mean zero and covariance function $\min (s, t)$. Moreover, for any $s \leq t$, the increment $B_{t}-B_{s}$ has the normal distribution $N(0, t-s)$. This implies that for any natural number $k$ we have

$$
\mathbb{E}\left(\left(B_{t}-B_{s}\right)^{2 k}\right)=\frac{(2 k) !}{2^{k} k !}(t-s)^{k} .
$$

Therefore, by Kolmogorov's continuity theorem, there exists a version of $B$ with Hölder-continuous trajectories of order $\gamma$ for any $\gamma<(k-1) /(2 k)$ on the interval $[0, T]$. This implies that the paths of this version of the process $B$ are $\gamma$-Hölder continuous on $[0, T]$ for any $\gamma<1 / 2$.

The mapping $P$ defined by (1.2) on cylinder sets can be uniquely extended to a probability measure on $\mathcal{F}$. This fact can be proved as a consequence of the existence of Brownian motion on $[0, T]$. 


\subsubsection{Wiener integral}

We next define the integral of square integrable functions with respect to the Brownian motion, known as the Wiener integral. We consider the set $\mathcal{E}_{0}$ of step functions

$$
\varphi_{t}=\sum_{j=0}^{n-1} a_{j} \mathbf{1}_{\left(t_{j}, t_{j+1}\right]}(t), \quad t \in[0, T]
$$

where $n \geq 1$ is an integer, $a_{0}, \ldots, a_{n-1} \in \mathbb{R}$, and $0=t_{0}<\cdots<t_{n} \leq T$. The Wiener integral of a step function $\varphi \in \mathcal{E}_{0}$ of the form (1.4) is defined by

$$
\int_{0}^{T} \varphi_{t} d B_{t}=\sum_{j=0}^{n-1} a_{j}\left(B_{t_{j+1}}-B_{t_{j}}\right)
$$

The mapping $\varphi \rightarrow \int_{0}^{T} \varphi_{t} d B_{t}$ from $\mathcal{E}_{0} \subset L^{2}([0, T])$ to $L^{2}(\Omega)$ is linear and isometric:

$$
\mathbb{E}\left(\left(\int_{0}^{T} \varphi_{t} d B_{t}\right)^{2}\right)=\sum_{j=0}^{n-1} a_{j}^{2}\left(t_{j+1}-t_{j}\right)=\int_{0}^{T} \varphi_{t}^{2} d t=\|\varphi\|_{L^{2}([0, T])}^{2} .
$$

The space $\mathcal{E}_{0}$ is a dense subspace of $L^{2}([0, T])$. Therefore, the mapping

$$
\varphi \rightarrow \int_{0}^{T} \varphi_{t} d B_{t}
$$

can be extended to a linear isometry between $L^{2}([0, T])$ and the Gaussian subspace of $L^{2}(\Omega)$ spanned by the Brownian motion. The random variable $\int_{0}^{T} \varphi_{t} d B_{t}$ is called the Wiener integral of $\varphi \in L^{2}([0, T])$ and is denoted by $B(\varphi)$. Observe that it is a Gaussian random variable with mean zero and variance $\|\varphi\|_{L^{2}([0, T])}^{2}$.

\subsubsection{The derivative operator}

Let $B=\left(B_{t}\right)_{t \in[0, T]}$ be a Brownian motion on the Wiener space $(\Omega, \mathcal{F}, P)$. Set $\mathfrak{H}=L^{2}([0, T])$, and for any $h \in \mathfrak{H}$, consider the Wiener integral

$$
B(h)=\int_{0}^{T} h(t) d B_{t}
$$

The Hilbert space $\mathfrak{H}$ plays a basic role in the definition of the derivative operator. In fact, the derivative of a random variable $F: \Omega \rightarrow \mathbb{R}$ takes values in $\mathfrak{H}$, and $\left(D_{t} F\right)_{t \in[0, T]}$ is a stochastic process in $L^{2}(\Omega ; \mathfrak{H})$. 
We start by defining the derivative in a dense subset of $L^{2}(\Omega)$. More precisely, consider the set $\mathcal{S}$ of smooth and cylindrical random variables of the form

$$
F=f\left(B\left(h_{1}\right), \ldots, B\left(h_{n}\right)\right),
$$

where $f \in C_{p}^{\infty}\left(\mathbb{R}^{n}\right)$ ( $f$ is infinitely differentiable and, together with all its partial derivatives, it has polynomial growth) and $h_{i} \in \mathfrak{H}$.

Definition 1.2.1. If $F \in \mathcal{S}$ is a smooth and cylindrical random variable of the form (1.5), the derivative operator $D F$ is the $\mathfrak{H}$-valued random variable defined by

$$
D_{t} F=\sum_{i=1}^{n} \frac{\partial f}{\partial x_{i}}\left(B\left(h_{1}\right), \ldots, B\left(h_{n}\right)\right) h_{i}(t) .
$$

For instance, $D(B(h))=h$ and $D\left(B_{t_{1}}\right)=\mathbf{1}_{\left[0, t_{1}\right]}$, for any $t_{1} \in[0, T]$.

The derivative operator can be interpreted as a directional derivative. Consider the Cameron-Martin space $H^{1} \subset \Omega$, which is is the set of functions of the form $\psi(t)=\int_{0}^{t} h(s) d s$, where $h \in \mathfrak{H}$. Then, for ant $h \in \mathfrak{H},\langle D F, h\rangle_{\mathfrak{H}}$ is the derivative of $F$ in the direction of $\int_{0}^{\cdot} h(s) d s$ :

$$
\langle D F, h\rangle_{\mathfrak{H}}=\int_{0}^{T} h_{t} D_{t} F d t=\left.\frac{d}{d \epsilon} F\left(\omega+\epsilon \int_{0}^{\cdot} h_{s} d s\right)\right|_{\epsilon=0} .
$$

For example, if $F=B_{t_{1}}$, then

$$
F\left(\omega+\epsilon \int_{0}^{\cdot} h_{s} d s\right)=\omega\left(t_{1}\right)+\epsilon \int_{0}^{t_{1}} h_{s} d s,
$$

so, the directional derivative $\int_{0}^{t_{1}} h_{s} d s$ coincides with $\langle D F, h\rangle_{\mathfrak{H}}$ because $D_{t} F=\mathbf{1}_{\left[0, t_{1}\right]}(t)$.

The operator $D$ defines a linear and unbounded operator from $\mathcal{S} \subset$ $L^{2}(\Omega)$ into $L^{2}(\Omega ; \mathfrak{H})$. Let us now introduce the divergence operator. Denote by $\mathcal{S}_{\mathfrak{H}}$ the class of smooth and cylindrical stochastic processes $u=\left(u_{t}\right)_{t \in[0, T]}$ of the form

$$
u_{t}=\sum_{j=1}^{n} F_{j} h_{j}(t)
$$

where $F_{j} \in \mathcal{S}$ and $h_{j} \in \mathfrak{H}$.

Definition 1.2.2. We define the divergence of an element $u$ of the form (1.6) as the random variable given by

$$
\delta(u)=\sum_{j=1}^{n} F_{j} B\left(h_{j}\right)-\sum_{j=1}^{n}\left\langle D F_{j}, h_{j}\right\rangle_{\mathfrak{H}} .
$$


In particular, for any $h \in \mathfrak{H}$ we have $\delta(h)=B(h)$.

As in the finite-dimensional case, the divergence is the adjoint of the derivative operator, as it is shown in the next proposition.

Proposition 1.2.1. Let $F \in \mathcal{S}$ and $u \in \mathcal{S}_{\mathfrak{H}}$. Then

$$
\mathbb{E}(F \delta(u))=\mathbb{E}\left(\langle D F, u\rangle_{\mathfrak{H}}\right) .
$$

Proof. We can assume that $F=f\left(B\left(h_{1}\right) \ldots, B\left(h_{n}\right)\right)$ and

$$
u=\sum_{j=1}^{n} g_{j}\left(B\left(h_{1}\right) \ldots, B\left(h_{n}\right)\right) h_{j},
$$

where $h_{1}, \ldots, h_{n}$ are orthonormal elements in $\mathfrak{H}$. In this case, the duality relationship reduces to the finite-dimensional case proved in Proposition 1.1.1.

We will make use of the notation $D_{h} F=\langle D F, h\rangle_{\mathfrak{H}}$ for any $h \in \mathfrak{H}$ and $F \in \mathcal{S}$. The following two properties are immediate consequence of the definitions and their proof is left as an exercise:

Heisenberg commutation relation: For ay $u \in \mathcal{S}_{\mathfrak{H}}$ and $h \in \mathfrak{H}$,

$$
D_{h}(\delta(u))=\delta\left(D_{h} u\right)+\langle h, u\rangle_{\mathfrak{H}} .
$$

Factorization: For any $F \in \mathcal{S}$ and $u \in \mathcal{S}_{\mathfrak{H}}$

$$
\delta(F u)=F \delta(u)-\langle D F, u\rangle_{\mathfrak{H}} .
$$

The following proposition provides a formula for the covariance of two divergences:

Proposition 1.2.2. Suppose that $u, v \in \mathcal{S}_{\mathfrak{H}}$. Then, we have

$$
\mathbb{E}(\delta(u) \delta(v))=\mathbb{E}\left(\langle u, v\rangle_{\mathfrak{H}}\right)+\mathbb{E}\left[\left\langle D u, D^{*} v\right\rangle_{\mathfrak{H}^{\otimes 2}}\right],
$$

where

$$
\left\langle D u, D^{*} v\right\rangle_{\mathfrak{H} \otimes 2}=\sum_{i, j=1}^{\infty} D_{e_{i}}\left\langle u, e_{j}\right\rangle_{\mathfrak{H}} D_{e_{j}}\left\langle v, e_{i}\right\rangle_{\mathfrak{H}},
$$

and $\left(e_{i}\right)_{i \geq 1}$ is a complete orthonormal system in $\mathfrak{H}$,

Property (1.10) can also be written as

$$
\mathbb{E}(\delta(u) \delta(v))=\mathbb{E}\left(\int_{0}^{T} u_{t} v_{t} d t\right)+\mathbb{E}\left(\int_{0}^{T} \int_{0}^{T} D_{s} u_{t} D_{t} v_{s} d s d t\right) .
$$


Proof of Proposition 1.2.2. To show property (1.10), using the duality formula (Proposition 1.2.1) and property (1.8), we write

$$
\begin{aligned}
\mathbb{E}(\delta(u) \delta(v)) & =\mathbb{E}\left(\langle v, D(\delta(u))\rangle_{\mathfrak{H}}\right) \\
& =\mathbb{E}\left(\sum_{i=1}^{\infty}\left\langle v, e_{i}\right\rangle_{\mathfrak{H}} D_{e_{i}}(\delta(u))\right) \\
& =\mathbb{E}\left(\sum_{i=1}^{\infty}\left\langle v, e_{i}\right\rangle_{\mathfrak{H}}\left(\left\langle u, e_{i}\right\rangle_{\mathfrak{H}}+\delta\left(D_{e_{i}} u\right)\right)\right) \\
& =\mathbb{E}\left(\langle u, v\rangle_{\mathfrak{H}}\right)+\mathbb{E}\left(\sum_{i, j=1}^{\infty} D_{e_{i}}\left\langle u, e_{j}\right\rangle_{\mathfrak{H}} D_{e_{j}}\left\langle v, e_{i}\right\rangle_{\mathfrak{H}}\right)
\end{aligned}
$$

This completes the proof.

\subsubsection{Sobolev spaces}

The next proposition will play a basic role in extending the derivative to suitable Sobolev spaces of random variables.

Proposition 1.2.3. The operator $D$ is closable from $L^{p}(\Omega)$ to $L^{p}(\Omega ; \mathfrak{H})$ for any $p \geq 1$.

Proof. Assume that a sequence $F_{N} \in \mathcal{S}$ satisfies, for some $p \geq 1$,

$$
F_{N} \stackrel{L^{p}(\Omega)}{\longrightarrow} 0 \text { and } \quad D F_{N} \stackrel{L^{p}(\Omega ; \mathfrak{H})}{\longrightarrow} \eta
$$

as $N \rightarrow \infty$. Then $\eta=0$. Indeed, for any $u=\sum_{j=1}^{N} G_{j} h_{j} \in \mathcal{S}_{\mathfrak{H}}$ such that $G_{j} B\left(h_{j}\right)$ and $D G_{j}$ are bounded, by the duality formula (Proposition 1.2.1), we obtain

$$
\begin{aligned}
\mathbb{E}\left(\langle\eta, u\rangle_{\mathfrak{H}}\right) & =\lim _{N \rightarrow \infty} \mathbb{E}\left(\left\langle D F_{N}, u\right\rangle_{\mathfrak{H}}\right) \\
& =\lim _{N \rightarrow \infty} \mathbb{E}\left(F_{N} \delta(u)\right)=0
\end{aligned}
$$

This implies that $\eta=0$, since the set of $u \in \mathcal{S}_{\mathfrak{H}}$ with the above properties is dense in $L^{p}(\Omega ; \mathfrak{H})$ for all $p \geq 1$.

We consider the closed extension of the derivative, which we also denote by $D$. The domain of this operator is defined by the following Sobolev spaces. For any $p \geq 1$, we denote by $\mathbb{D}^{1, p}$ the closure of $\mathcal{S}$ with respect to the seminorm

$$
\|F\|_{1, p}=\left(\mathbb{E}\left(|F|^{p}\right)+\mathbb{E}\left(\left|\int_{0}^{T}\left(D_{t} F\right)^{2} d t\right|^{p / 2}\right)\right)^{1 / p} .
$$


In particular, $F$ belongs to $\mathbb{D}^{1, p}$ if and only if there exists a sequence $F_{n} \in \mathcal{S}$ such that

$$
F_{n} \stackrel{L^{p}(\Omega)}{\longrightarrow} F \text { and } D F_{n} \stackrel{L^{p}(\Omega ; \mathfrak{H})}{\longrightarrow} D F
$$

as $n \rightarrow \infty$. For $p=2$, the space $\mathbb{D}^{1,2}$ is a Hilbert space with scalar product

$$
\langle F, G\rangle_{1,2}=\mathbb{E}(F G)+\mathbb{E}\left(\int_{0}^{T} D_{t} F D_{t} G d t\right) .
$$

In the same way we can introduce spaces $\mathbb{D}^{1, p}(\mathfrak{H})$ by taking the closure of $\mathcal{S}_{\mathfrak{H}}$. The corresponding seminorm is denoted by $\|\cdot\|_{1, p, \mathfrak{H}}$.

\subsubsection{Chain rule}

The Malliavin derivative satisfies the following chain rule.

Proposition 1.2.4. Let $\varphi: \mathbb{R} \rightarrow \mathbb{R}$ be a continuous differentiable function such that $\left|\varphi^{\prime}(x)\right| \leq C\left(1+|x|^{\alpha}\right)$ for some $\alpha \geq 0$. Let $F \in \mathbb{D}^{1, p}$ for some $p \geq \alpha+1$. Then, $\varphi(F)$ belongs to $\mathbb{D}^{1, q}$, where $q=p /(\alpha+1)$, and

$$
D(\varphi(F))=\varphi^{\prime}(F) D F .
$$

Proof. Notice that $|\varphi(x)| \leq C^{\prime}\left(1+|x|^{\alpha+1}\right)$, for some constant $C^{\prime}$, which implies that $\varphi(F) \in L^{q}(\Omega)$ and, by Hölder's inequality, $\varphi^{\prime}(F) D F \in$ $L^{q}(\Omega ; \mathfrak{H})$. Then, to show the proposition it suffices to approximate $F$ by smooth and cylindrical random variables, and $\varphi$ by $\varphi * \alpha_{n}$, where $\alpha_{n}$ is an approximation to the identity.

\subsubsection{Domain of the divergence in $L^{2}(\Omega)$}

We next define the domain of the divergence operator. We identify the Hilbert space $L^{2}(\Omega ; \mathfrak{H})$ with $L^{2}(\Omega \times[0, T])$.

Definition 1.2.3. The domain of the divergence operator $\operatorname{Dom} \delta$ in $L^{2}(\Omega)$ is the set of processes $u \in L^{2}(\Omega \times[0, T])$ such that there exists a constant $c_{u}$ satisfying

$$
\left|\mathbb{E}\left(\langle D F, u\rangle_{\mathfrak{H}}\right)\right| \leq c_{u}\|F\|_{2}
$$

for all $F \in \mathbb{D}^{1,2}$.

By Riesz theorem, if $u \in \operatorname{Dom} \delta$, there exists a random variable $\delta(u) \in L^{2}(\Omega)$ satisfying

$$
\mathbb{E}\left(\langle D F, u\rangle_{\mathfrak{H}}\right)=\mathbb{E}(\delta(u) F),
$$

for any $F \in \mathbb{D}^{1,2}$. Observe, that by the duality formula in Proposition $1.2 .1, \mathcal{S}_{\mathfrak{H}} \subset \operatorname{Dom} \delta$ and for $u \in \mathcal{S}_{\mathfrak{H}}, \delta(u)$ is given by (1.7). 
Notice that $\delta$ is a linear operator such that $\mathbb{E}(\delta(u))=0$. Moreover, $\delta$ is closed; that is, if the sequence $u_{n} \in \operatorname{Dom} \delta$ satisfies

$$
u_{n} \stackrel{L^{2}(\Omega ; \mathfrak{H})}{\longrightarrow} u \text { and } \delta\left(u_{n}\right) \stackrel{L^{2}(\Omega)}{\longrightarrow} G
$$

as $n \rightarrow \infty$, then $u$ belongs to $\operatorname{Dom} \delta$ and $\delta(u)=G$.

From Proposition 1.2.2 it follows that $\mathbb{D}^{1,2}(\mathfrak{H}) \subset$ Dom $\delta$ and for $u, v \in$ $\mathbb{D}^{1,2}(\mathfrak{H}) \subset$ Dom $\delta(1.10)$ holds, which implies

$$
\mathbb{E}\left(\delta(u)^{2}\right) \leq \mathbb{E}\left(\int_{0}^{T} u_{t}^{2} d t\right)+\mathbb{E}\left(\int_{0}^{T} \int_{0}^{T}\left(D_{s} u_{t}\right)^{2} d s d t\right)=\|u\|_{1,2, \mathfrak{H}}^{2} .
$$

Properties (1.8) and property (1.9) can be extended to random variables in suitable Sobolev spaces. More precisely, property (1.8) holds if $u \in \mathbb{D}^{1,2}(\mathfrak{H})$ and $D_{h} u \in \operatorname{Dom} \delta$ and property (1.9) holds if $F \in \mathbb{D}^{1,2}$, $F u \in L^{2}(\Omega ; \mathfrak{H}), u \in \operatorname{Dom} \delta$, and the right-hand side is square integrable.

\subsubsection{Iterated derivatives}

We can also introduce iterated derivatives and the corresponding Sobolev spaces. The $k$ th derivative $D^{k} F$ of a random variable $F \in \mathcal{S}$ is the $k$ parameter process obtained by iteration:

$$
D_{t_{1}, \ldots t_{k}}^{k} F=\sum_{i_{1}, \ldots, i_{k}=1}^{n} \frac{\partial^{k} f}{\partial x_{i_{1}} \cdots \partial x_{i_{k}}}\left(B\left(h_{1}\right), \ldots, B\left(h_{n}\right)\right) h_{i_{1}}\left(t_{1}\right) \cdots h_{i_{k}}\left(t_{k}\right)
$$

For any $p \geq 1$, the operator $D^{k}$ is closable from $L^{p}(\Omega)$ into $L^{p}\left(\Omega ; \mathfrak{H}^{\otimes k}\right)$, and we denote by $\mathbb{D}^{k, p}$ the closure of $\mathcal{S}$ with respect to the seminorm

$$
\|F\|_{k, p}=\left(\mathbb{E}\left(|F|^{p}\right)+\mathbb{E}\left(\sum_{j=1}^{k}\left|\int_{[0, T]^{j}}\left(D_{t_{1}, \ldots, t_{j}}^{j} F\right)^{2} d t_{1} \cdots d t_{j}\right|^{p / 2}\right)\right)^{1 / p} .
$$

For any $k \geq 1$, we set $\mathbb{D}^{k, \infty}:=\cap_{p \geq 2} \mathbb{D}^{k, p}, \mathbb{D}^{\infty, 2}:=\cap_{k \geq 1} \mathbb{D}^{k, 2}$, and $\mathbb{D}^{\infty}:=\cap_{k \geq 1} \overline{\mathbb{D}}^{k, \infty}$. Similarly, we can introduce the spaces $\mathbb{D}^{k, p}(\mathfrak{H})$.

\subsubsection{Continuity of the divergence}

The following theorem is a consequence of Meyer's inequalities:

Theorem 1.2.4. For any $p>1$ and $u \in \mathbb{D}^{1, p}(\mathfrak{H})$,

$$
\mathbb{E}\left(|\delta(u)|^{p}\right) \leq c_{p}\left(\mathbb{E}\left(\|D u\|_{\mathfrak{H} \otimes \mathfrak{H}}^{p}\right)+\mathbb{E}\left(\|u\|_{\mathfrak{H}}^{p}\right)\right)
$$


A proof based on the boundedness in $L^{p}(\mathbb{R})$ of the Hilbert transform was given by Pisier [26]. We can introduce the iterated divergence and it coincides with the adjoint of the iterated derivative:

$$
\mathbb{E}\left(\delta^{k}(u) F\right)=\mathbb{E}\left(\left\langle u, D^{k} F\right\rangle_{\mathfrak{H} \otimes k}\right) .
$$

The above estimate can be generalized as follows: for any integer $k \geq 1$ and real $p>1$, we have

$$
\mathbb{E}\left(\left|\delta^{k}(u)\right|^{p}\right) \leq c_{p, k} \sum_{\ell=0}^{k} \mathbb{E}\left(\left\|D^{\ell} u\right\|_{\mathfrak{H} \otimes(k+\ell)}^{p}\right) .
$$

This means that $\delta^{k}$ is continuous from $\mathbb{D}^{k, p}\left(\mathfrak{H}^{\otimes k}\right)$ to $L^{p}(\Omega)$ for $p>1$.

\subsubsection{The divergence as a stochastic integral}

The Malliavin derivative is a local operator in the following sense. Let $[a, b] \subset \mathbb{R}_{+}$be fixed. We denote by $\mathcal{F}_{[a, b]}$ the $\sigma$-field generated by the random variables $\left\{B_{s}-B_{a}, s \in[a, b]\right\}$.

Lemma 1.2.5. Let $F$ be a random variable in $\mathbb{D}^{1,2} \cap L^{2}\left(\Omega, \mathcal{F}_{[a, b]}, P\right)$. Then $D_{t} F=0$ for almost all $(\omega, t) \in \Omega \times[a, b]^{c}$.

Proof. If $F$ belongs to $\mathcal{S} \cap L^{2}\left(\Omega, \mathcal{F}_{[a, b]}, P\right)$ then this property is clear. The general case follows by approximation.

The following result says that the divergence operator is an extension of Itô's integral. For any $t \geq 0$ we denote by $\mathcal{F}_{t}$ the $\sigma$-algebra generated by the null sets and the random variables $\left\{B_{s}, s \in[0, t]\right\}$.

Theorem 1.2.6. Any process $u$ in $L^{2}(\Omega \times[0, T])$ which is adapted (for each $t \geq 0, u_{t}$ is $\mathcal{F}_{t}$-measurable) belongs to $\operatorname{Dom} \delta$ and $\delta(u)$ coincides with Itô's stochastic integral

$$
\delta(u)=\int_{0}^{T} u_{t} d B_{t}
$$

Proof. Consider a simple process $u$ of the form

$$
u_{t}=\sum_{j=0}^{n-1} \phi_{j} \mathbf{1}_{\left(t_{j}, t_{j+1}\right]}(t)
$$

where $0 \leq t_{0}<t_{1}<\cdots<t_{n} \leq T$ and the random variables $\phi_{j} \in \mathcal{S}$ are $\mathcal{F}_{t_{j}}$-measurable. Then $\delta(u)$ coincides with the Itô integral of $u$ because, by $(1.9)$,

$$
\delta(u)=\sum_{j=0}^{n-1} \phi_{j}\left(B_{t_{j+1}}-B_{t_{j}}\right)-\sum_{j=0}^{n-1} \int_{t_{j}}^{t_{j+1}} D_{t} \phi_{j} d t=\sum_{j=0}^{n-1} \phi_{j}\left(B_{t_{j+1}}-B_{t_{j}}\right),
$$


taking into account that $D_{t} \phi_{j}=0$ if $t>t_{j}$ by Lemma 1.2.5. Then the result follows by approximating any adapted and square integrable process by simple processes, and approximating any $\phi_{j} \in L^{2}\left(\Omega, \mathcal{F}_{t_{j}}, P\right)$ by $\mathcal{F}_{t_{j}}$-measurable smooth and cylindrical random variables.

If $u$ is not adapted, $\delta(u)$ coincides with an anticipating stochastic integral introduced by Skorohod (see [27]). Using techniques of Malliavin calculus, Nualart and Pardoux [21] developed a stochastic calculus for the Skorohod integral.

If $u$ and $v$ are adapted then, for $s<t, D_{t} v_{s}=0$ and, for $s>t, D_{s} u_{t}=0$. As a consequence, property (1.10) leads to the isometry property of Itô's integral for adapted processes $u, v \in \mathbb{D}^{1,2}(\mathfrak{H})$ :

$$
\mathbb{E}(\delta(u) \delta(v))=\mathbb{E}\left(\int_{0}^{T} u_{t} v_{t} d t\right) .
$$

If $u$ is an adapted process in $\mathbb{D}^{1,2}(\mathfrak{H})$ then, from property (1.8), we obtain

$$
D_{t}\left(\int_{0}^{T} u_{s} d B_{s}\right)=u_{t}+\int_{t}^{T} D_{t} u_{s} d B_{s},
$$

because $D_{t} u_{s}=0$ if $t>s$.

\subsubsection{Clark-Ocone formula}

Let $B=\left(B_{t}\right)_{t \in[0, T]}$ be a Brownian motion on the Wiener space $(\Omega, \mathcal{F}, P)$, equipped with its Brownian filtration $\left(\mathcal{F}_{t}\right)_{t \in[0, T]}$. The next result expresses the integrand of the integral representation theorem of a square integrable random variable in terms of the conditional expectation of its Malliavin derivative.

Theorem 1.2.7 (Clark-Ocone formula). Let $F \in \mathbb{D}^{1,2} \cap L^{2}(\Omega, \mathcal{F}, P)$. Then $F$ admits the following representation:

$$
F=\mathbb{E}(F)+\int_{0}^{T} \mathbb{E}\left(D_{t} F \mid \mathcal{F}_{t}\right) d B_{t} .
$$

Proof. By the Itô integral representation theorem, there exists a unique adapted process $u \in L^{2}(\Omega \times[0, T])$ such that $F \in L^{2}(\Omega, \mathcal{F}, P)$ admits the stochastic integral representation

$$
F=\mathbb{E}(F)+\int_{0}^{T} u_{t} d B_{t}
$$

It suffices to show that $u_{t}=\mathbb{E}\left(D_{t} F \mid \mathcal{F}_{t}\right)$ for almost all $(\omega, t) \in \Omega \times[0, T]$. Consider an adapted and square integrable process $v$. On the one hand, 
the isometry property yields

$$
\mathbb{E}(\delta(v) F)=\int_{0}^{T} \mathbb{E}\left(v_{s} u_{s}\right) d s .
$$

On the other hand, by the duality relationship (Proposition 1.2.1), and taking into account that $v$ is adapted,

$$
\mathbb{E}(\delta(v) F)=\mathbb{E}\left(\int_{0}^{T} v_{t} D_{t} F d t\right)=\int_{0}^{T} \mathbb{E}\left(v_{s} \mathbb{E}\left(D_{t} F \mid \mathcal{F}_{t}\right)\right) d t .
$$

Therefore, $u_{t}=\mathbb{E}\left(D_{t} F \mid \mathcal{F}_{t}\right)$ for almost all $(\omega, t) \in \Omega \times[0, T]$, which concludes the proof.

Consider the following simple examples of the application of this formula.

Example 1.2.8. Suppose that $F=B_{t}^{3}$. Then $D_{s} F=3 B_{t}^{2} \mathbf{1}_{[0, t]}(s)$ and

$$
\mathbb{E}\left(D_{s} F \mid \mathcal{F}_{s}\right)=3 \mathbb{E}\left(\left(B_{t}-B_{s}+B_{s}\right)^{2} \mid \mathcal{F}_{s}\right)=3\left(t-s+B_{s}^{2}\right) .
$$

Therefore

$$
B_{t}^{3}=3 \int_{0}^{t}\left(t-s+B_{s}^{2}\right) d B_{s} .
$$

This formula should be compared with Itô's formula,

$$
B_{t}^{3}=3 \int_{0}^{t} B_{s}^{2} d B_{s}+3 \int_{0}^{t} B_{s} d s .
$$

Notice that equation (1.13) contains only a stochastic integral but it is not a martingale, because the integrand depends on $t$, whereas (1.14) contains two terms and one is a martingale. Moreover, the integrand in (1.13) is unique.

Example 1.2.9. Consider the Brownian motion local time $\left(L_{t}^{x}\right)_{t \geq 0, x \in \mathbb{R}}$. For any $\varepsilon>0$, we set

$$
p_{\varepsilon}(x)=(2 \pi \varepsilon)^{-1 / 2} e^{-x^{2} /(2 \varepsilon)} .
$$

We have that, as $\epsilon \rightarrow 0$,

$$
F_{\varepsilon}=\int_{0}^{t} p_{\varepsilon}\left(B_{s}-x\right) d s \stackrel{L^{2}(\Omega)}{\longrightarrow} L_{t}^{x} .
$$

Applying the derivative operator yields

$$
D_{r} F_{\epsilon}=\int_{0}^{t} p_{\epsilon}^{\prime}\left(B_{s}-x\right) D_{r} B_{s} d s=\int_{r}^{t} p_{\epsilon}^{\prime}\left(B_{s}-x\right) d s .
$$


Thus

$$
\begin{aligned}
\mathbb{E}\left(D_{r} F_{\epsilon} \mid \mathcal{F}_{r}\right) & =\int_{r}^{t} \mathbb{E}\left(p_{\epsilon}^{\prime}\left(B_{s}-B_{r}+B_{r}-x\right) \mid \mathcal{F}_{r}\right) d s \\
& =\int_{r}^{t} p_{\epsilon+s-r}^{\prime}\left(B_{r}-x\right) d s
\end{aligned}
$$

As a consequence, taking the limit as $\epsilon \rightarrow 0$, we obtain the following integral representation of the Brownian local time:

$$
L_{t}^{x}=\mathbb{E}\left(L_{t}^{x}\right)+\int_{0}^{t} \varphi\left(t-r, B_{r}-x\right) d B_{r},
$$

where

$$
\varphi(r, y)=\int_{0}^{r} p_{s}^{\prime}(y) d s
$$

\subsubsection{Isonormal Gaussian processes}

So far, we have developed the Malliavin calculus with respect to Brownian motion. In this case, the Wiener integral $B(h)=\int_{0}^{T} h(t) d B_{t}$ gives rise to a centered Gaussian family indexed by the Hilbert space $\mathfrak{H}=L^{2}([0, T])$. More generally, consider a separable Hilbert space $\mathfrak{H}$ with scalar product $\langle\cdot, \cdot\rangle_{\mathfrak{H}}$. An isonormal Gaussian process is a centered Gaussian family $\mathcal{H}_{1}=\{W(h), h \in \mathfrak{H}\}$ of random variables defined in some probability space $(\Omega, \mathcal{F}, P)$, satisfying

$$
\mathbb{E}(W(h) W(g))=\langle h, g\rangle_{\mathfrak{H}},
$$

for any $h, g \in \mathfrak{H}$. Observe that $\mathcal{H}_{1}$ is a Gaussian subspace of $L^{2}(\Omega)$.

The Malliavin calculus can be developed in the framework of an isonormal Gaussian process, and all the notions and properties that do not depend on the fact that $\mathfrak{H}=L^{2}([0, T])$ can be extended to this more general context.

\subsection{Multiple stochastic integrals. Wiener chaos}

In this section we present the Wiener chaos expansion, which provides an orthogonal decomposition of random variables in $L^{2}(\Omega)$ in terms of multiple stochastic integrals. We then compute the derivative and the divergence operators on the Wiener chaos expansion. 


\subsubsection{Hermite polynomials}

Consider the probability space $(\mathbb{R}, \mathcal{B}(\mathbb{R}), \gamma)$, where $\gamma=N(0,1)$ is the standard Gaussian probability on $\mathbb{R}$ with density

$$
p(x)=\frac{1}{\sqrt{2 \pi}} e^{-x^{2} / 2}, \quad x \in \mathbb{R} .
$$

Recall the basic differential operators:

- Derivative operator: $D f(x)=f^{\prime}(x)$.

- Divergence operator: $\delta f(x)=x f(x)-f^{\prime}(x)$.

They satisfy the following Heisenberg's commutation relation for $f \in$ $C^{2}(\mathbb{R})$ :

$$
(D \delta-\delta D) f=f .
$$

Define the Hermite polynomials as follows. $H_{0}(x)=1$, and for $n \geq 1$ put $H_{n}(x)=\delta^{n} 1$. In particular, for $n=1,2,3$, we have

$$
\begin{aligned}
& H_{1}(x)=\delta 1=x \\
& H_{2}(x)=\delta x=x^{2}-1 \\
& H_{3}(x)=\delta\left(x^{2}-1\right)=x^{3}-3 x .
\end{aligned}
$$

The Hermite polynomials satisfy the following properties:

1. Formula for the derivative:

$$
H_{m}^{\prime}=m H_{m-1}
$$

In fact, using induction and the Heisenberg commutation relation, we can write

$$
\begin{aligned}
H_{m}^{\prime} & =D\left(\delta^{m} 1\right)=(D \delta)\left(\delta^{m-1} 1\right)=\delta D\left(\delta^{m-1} 1\right)+\delta^{m-1} 1 \\
& =\delta D H_{m-1}+H_{m-1}=\delta(m-1) H_{m-2}+H_{m-1}=m H_{m-1}
\end{aligned}
$$

2. Recursion formula:

$$
H_{m+1}(x)=x H_{m}(x)-H_{m}^{\prime}(x)=x H_{m}(x)-m H_{m-1}(x) .
$$

3. The sequence of normalized Hermite polynomials $\left\{\frac{1}{\sqrt{n !}} H_{n}, n \geq 0\right\}$ form a complete orthonormal system of functions in the Hilbert space $L^{2}(\mathbb{R}, \gamma)$. 
Proof. For $n, m \geq 0$, we can write

$$
\int_{\mathbb{R}} H_{n}(x) H_{m}(x) p(x) d x= \begin{cases}n ! & \text { if } n=m \\ 0 & \text { if } n \neq m\end{cases}
$$

Indeed, using the properties of Hermite polynomials, we obtain

$$
\begin{aligned}
\int_{\mathbb{R}} H_{n}(x) H_{m}(x) p(x) d x & =\int_{\mathbb{R}} H_{n}(x) \delta^{m} 1(x) p(x) d x \\
& =\int_{\mathbb{R}} H_{n}^{\prime}(x) \delta^{m-1} 1(x) p(x) d x \\
& =n \int_{\mathbb{R}} H_{n-1}(x) H_{m-1}(x) p(x) d x
\end{aligned}
$$

Then, we obtain (1.16) by iteration.

To show completeness, it suffices to prove that if $f \in L^{2}(\mathbb{R}, \gamma)$ is orthogonal to all Hermite polynomials, then $f=0$. Because the leading coefficient of $H_{n}(x)$ is 1 , we have that $f$ is orthogonal to all monomials $x^{n}$. As a consequence, for all $t \in \mathbb{R}$,

$$
\int_{\mathbb{R}} f(x) e^{i t x} p(x) d x=\sum_{n=0}^{\infty} \frac{(i t)^{n}}{n !} \int_{\mathbb{R}} f(x) x^{n} p(x) d x=0 .
$$

Notice that we can commute the integral and the series because

$$
\begin{gathered}
\sum_{n=0}^{\infty} \int_{\mathbb{R}} \frac{|t x|^{n}}{n !}|f(x)| p(x) d x=\int_{\mathbb{R}} e^{|t x|}|f(x)| p(x) d x \\
\leq\left[\int_{\mathbb{R}} f^{2}(x) p(x) d x \int_{\mathbb{R}} e^{2|t x|} p(x) d x\right]^{\frac{1}{2}}<\infty
\end{gathered}
$$

Therefore, the Fourier transform of $f p$ is zero, so $f p=0$, which implies $f=0$. This completes the proof.

4. Series expansion: For each $a \in \mathbb{R}$, we have the following series expansion, which will play an important role

$$
\sum_{n=0}^{\infty} \frac{a^{n}}{n !} H_{n}(x)=e^{a x-\frac{a^{2}}{2}}
$$

Proof of (2.7): Taking into account that $H_{n}=\delta^{n} 1$ and that $\delta^{n}$ is the 
adjoint of $D^{n}$, we obtain

$$
\begin{aligned}
e^{a x} & =\sum_{n=0}^{\infty} \frac{1}{n !}\left\langle e^{a \cdot}, H_{n}\right\rangle_{L^{2}(\mathbb{R}, \gamma)} H_{n}(x) \\
& =\sum_{n=0}^{\infty} \frac{1}{n !}\left\langle e^{a \cdot}, \delta^{n} 1\right\rangle_{L^{2}(\mathbb{R}, \gamma)} H_{n}(x) \\
& =\sum_{n=0}^{\infty} \frac{1}{n !}\left\langle D^{n}\left(e^{a \cdot}\right), 1\right\rangle_{L^{2}(\mathbb{R}, \gamma)} H_{n}(x) \\
& =\sum_{n=0}^{\infty} \frac{a^{n}}{n !}\left\langle e^{a \cdot}, 1\right\rangle_{L^{2}(\mathbb{R}, \gamma)} H_{n}(x) .
\end{aligned}
$$

Finally,

$$
\left\langle e^{a \cdot}, 1\right\rangle_{L^{2}(\mathbb{R}, \gamma)}=\frac{1}{\sqrt{2 \pi}} \int_{\mathbb{R}} e^{a x-\frac{x^{2}}{2}} d x=e^{\frac{a^{2}}{2}} .
$$

and (1.17) holds true.

\subsubsection{Multiple Stochastic Integrals}

Recall that $B=\left(B_{t}\right)_{t \in[0, T]}$ is a Brownian motion defined on the Wiener space $(\Omega, \mathcal{F}, P)$. Let $L_{s}^{2}\left([0, T]^{n}\right)$ be the space of symmetric square integrable functions $f:[0, T]^{n} \rightarrow \mathbb{R}$. If $f:[0, T]^{n} \rightarrow \mathbb{R}$, we define its symmetrization by

$$
\tilde{f}\left(t_{1}, \ldots, t_{n}\right)=\frac{1}{n !} \sum_{\sigma} f\left(t_{\sigma(1)}, \ldots, t_{\sigma(n)}\right),
$$

where the sum runs over all permutations $\sigma$ of $\{1,2, \ldots, n\}$. Observe that

$$
\|\tilde{f}\|_{L^{2}\left([0, T]^{n}\right)} \leq\|f\|_{L^{2}\left([0, T]^{n}\right)} .
$$

Definition 1.3.1. The multiple stochastic integral of $f \in L_{s}^{2}\left([0, T]^{n}\right)$ is defined as the iterated Itô stochastic integral

$$
I_{n}(f)=n ! \int_{0}^{T} \int_{0}^{t_{n}} \cdots \int_{0}^{t_{2}} f\left(t_{1}, \ldots, t_{n}\right) d B_{t_{1}} \cdots d B_{t_{n}} .
$$

Note that if $f \in L^{2}([0, T]), I_{1}(f)=B(f)$ is the Wiener integral of $f$. If $f \in L^{2}\left([0, T]^{n}\right)$ is not necessarily symmetric, we define

$$
I_{n}(f)=I_{n}(\tilde{f}) .
$$

Using the properties of Itô's stochastic integral, one can easily check the following isometry property: for all $n, m \geq 1, f \in L^{2}\left([0, T]^{n}\right)$, and 
$g \in L^{2}\left([0, T]^{m}\right)$

$$
\mathbb{E}\left(I_{n}(f) I_{m}(g)\right)= \begin{cases}0 & \text { if } n \neq m \\ n !\langle\tilde{f}, \tilde{g}\rangle_{L^{2}\left([0, T]^{n}\right)} & \text { if } n=m\end{cases}
$$

Next, we want to compute the product of two multiple integrals. Let $f \in L_{s}^{2}\left([0, T]^{n}\right)$ and $g \in L_{s}^{2}\left([0, T]^{m}\right)$. For any $r=0, \ldots, n \wedge m$, we define the contraction of $f$ and $g$ of order $r$ to be the element of $L^{2}\left([0, T]^{n+m-2 r}\right)$ defined by

$$
\begin{aligned}
(f & \left.\otimes_{r} g\right)\left(t_{1}, \ldots, t_{n-r}, s_{1}, \ldots, s_{m-r}\right) \\
& =\int_{[0, T]^{r}} f\left(t_{1}, \ldots, t_{n-r}, x_{1}, \ldots, x_{r}\right) g\left(s_{1}, \ldots, s_{m-r}, x_{1}, \ldots, x_{r}\right) d x_{1} \cdots d x_{r} .
\end{aligned}
$$

We denote by $f \tilde{\otimes}_{r} g$ the symmetrization of $f \otimes_{r} g$. Then, the product of two multiple stochastic integrals satisfies the following formula:

$$
I_{n}(f) I_{m}(g)=\sum_{r=0}^{n \wedge m} r !\left(\begin{array}{c}
n \\
r
\end{array}\right)\left(\begin{array}{c}
m \\
r
\end{array}\right) I_{n+m-2 r}\left(f \otimes_{r} g\right)
$$

The next result gives the relation between multiple stochastic integrals and Hermite polynomials.

Proposition 1.3.1. For any $g \in L^{2}([0, T])$, we have

$$
I_{n}\left(g^{\otimes n}\right)=\|g\|_{L^{2}([0, T])}^{n} H_{n}\left(\frac{B(g)}{\|g\|_{L^{2}([0, T])}}\right),
$$

where $g^{\otimes n}\left(t_{1}, \ldots, t_{n}\right)=g\left(t_{1}\right) \cdots g\left(t_{n}\right)$.

Proof. We can assume that $\|g\|_{L^{2}([0, T])}=1$. Fix $a \in \mathbb{R}$ and set

$$
M_{t}=\exp \left(a \int_{0}^{t} g_{s} d B_{s}-\frac{1}{2} a^{2} \int_{0}^{t} g_{s}^{2} d s\right) .
$$

One one hand, we have, using (1.17),

$$
M_{T}=e^{a \int_{0}^{T} g_{s} d B_{s}-\frac{1}{2} a^{2}}=\sum_{n=0}^{\infty} \frac{a^{n}}{n !} H_{n}\left(\int_{0}^{T} g_{t} d B_{t}\right)
$$


On the other hand, using Itô's formula, we obtain

$$
\begin{aligned}
M_{T} & =1+\int_{0}^{T} a M_{s} g_{s} d B_{s} \\
& =1+a I_{1}(g)+a^{2} \int_{0}^{T} g_{s} \int_{0}^{s} M_{v} g_{v} d B_{v} \\
& =1+a I_{1}(g)+a^{2} \int_{0}^{T} g_{s} \int_{0}^{s} g_{v} d B_{v}+a^{3} \int_{0}^{T} g_{s} \int_{0}^{s} M_{v} g_{v} d B_{v} \\
& =\sum_{n=0}^{\infty} \frac{a^{n}}{n !} I_{n}\left(g^{\otimes n}\right) .
\end{aligned}
$$

Comparing both expansions yields the desired result.

\subsubsection{Wiener chaos expansion}

The next result is the Wiener chaos expansion.

Theorem 1.3.2. Every $F \in L^{2}(\Omega)$ can be uniquely expanded into a sum of multiple stochastic integrals as follows:

$$
F=\mathbb{E}(F)+\sum_{n=1}^{\infty} I_{n}\left(f_{n}\right),
$$

where $f_{n} \in L_{s}^{2}\left([0, T]^{n}\right)$.

For any $n \geq 1$, we denote by $\mathcal{H}_{n}$ the closed subspace of $L^{2}(\Omega)$ formed by all multiple stochastic integrals of order $n$. For $n=0, \mathcal{H}_{0}$ is the space of constants. Observe that $\mathcal{H}_{1}$ coincides with the Gaussian space $\left\{B(f), f \in L^{2}([0, T])\right\}$. Then Theorem 1.3 .2 can be reformulated by saying that we have the orthogonal decomposition

$$
L^{2}(\Omega)=\oplus_{n=0}^{\infty} \mathcal{H}_{n}
$$

Proof of Theorem 1.3.2. It suffices to show that if a random variable $G \in L^{2}(\Omega)$ is orthogonal to $\oplus_{n=0}^{\infty} \mathcal{H}_{n}$ then $G=0$. This assumption implies that $G$ is orthogonal to all random variables of the form $B(g)^{k}$, where $g \in L^{2}([0, T]), k \geq 0$. This in turn implies that $G$ is orthogonal to all the exponentials $\exp (B(h))$, which form a total set in $L^{2}(\Omega)$. So $G=0$.

\subsubsection{Derivative operator on the Wiener chaos}

Let us compute the derivative of a multiple stochastic integral.

Proposition 1.3.2. Let $f \in L_{s}^{2}\left([0, T]^{n}\right)$. Then $I_{n}(f) \in \mathbb{D}^{1,2}$ and

$$
D_{t} I_{n}(f)=n I_{n-1}(f(\cdot, t)) \text {. }
$$


Proof. Assume that $f=g^{\otimes n}$, with $\|g\|_{L^{2}([0, T])}=1$. Then, using Proposition 1.3.1 and the properties of Hermite polynomials, we have

$$
\begin{aligned}
D_{t} I_{n}(f) & =D_{t}\left(H_{n}(B(g))\right)=H_{n}^{\prime}(B(g)) D_{t}(B(g))=n H_{n-1}(B(g)) g(t) \\
& =n g(t) I_{n-1}\left(g^{\otimes(n-1)}\right)=n I_{n-1}(f(\cdot, t)) .
\end{aligned}
$$

The general case follows using linear combinations and a density argument. This finishes the proof.

Moreover, applying (1.18), we have

$$
\begin{aligned}
\mathbb{E}\left(\int_{0}^{T}\left(D_{t} I_{n}(f)\right)^{2} d t\right) & =n^{2} \int_{0}^{T} \mathbb{E}\left(I_{n-1}(f(\cdot, t))^{2}\right) d t \\
& =n^{2}(n-1) ! \int_{0}^{T}\|f(\cdot, t)\|_{L^{2}\left([0, T]^{n-1}\right)}^{2} d t \\
& =n n !\|f\|_{L^{2}\left([0, T]^{n}\right)}^{2} \\
& =n \mathbb{E}\left(I_{n}(f)^{2}\right) .
\end{aligned}
$$

As a consequence of Proposition 1.3.2 and (1.20), we deduce the following result.

Proposition 1.3.3. Let $F \in L^{2}(\Omega)$ with Wiener chaos expansion $F=$ $\sum_{n=0}^{\infty} I_{n}\left(f_{n}\right)$. Then $F \in \mathbb{D}^{1,2}$ if and only if

$$
\mathbb{E}\left(\|D F\|_{\mathfrak{H}}^{2}\right)=\sum_{n=1}^{\infty} n n !\left\|f_{n}\right\|_{L^{2}\left([0, T]^{n}\right)}^{2}<\infty,
$$

and in this case

$$
D_{t} F=\sum_{n=1}^{\infty} n I_{n-1}\left(f_{n}(\cdot, t)\right)
$$

Similarly, if $k \geq 2$, one can show that $F \in \mathbb{D}^{k, 2}$ if and only if

$$
\sum_{n=1}^{\infty} n^{k} n !\left\|f_{n}\right\|_{L^{2}\left([0, T]^{n}\right)}^{2}<\infty
$$

and in this case

$$
D_{t_{1}, \ldots, t_{k}}^{k} F=\sum_{n=k}^{\infty} n(n-1) \cdots(n-k+1) I_{n-k}\left(f_{n}\left(\cdot, t_{1}, \ldots, t_{k}\right)\right),
$$

where the series converges in $L^{2}\left(\Omega \times[0, T]^{k}\right)$. As a consequence, if $F \in \mathbb{D}^{\infty, 2}$ then the following formula, due to Stroock, allows us to compute explicitly the kernels in the Wiener chaos expansion of $F$ :

$$
f_{n}=\frac{1}{n !} \mathbb{E}\left(D^{n} F\right) \text {. }
$$


Example 1.3.3. Consider $F=B_{1}^{3}$. Then

$$
\begin{aligned}
f_{1}\left(t_{1}\right) & =\mathbb{E}\left(D_{t_{1}} B_{1}^{3}\right)=3 \mathbb{E}\left(B_{1}^{2}\right) \mathbf{1}_{[0,1]}\left(t_{1}\right)=3 \mathbf{1}_{[0,1]}\left(t_{1}\right), \\
f_{2}\left(t_{1}, t_{2}\right) & =\frac{1}{2} \mathbb{E}\left(D_{t_{1}, t_{2}}^{2} B_{1}^{3}\right)=3 \mathbb{E}\left(B_{1}\right) \mathbf{1}_{[0,1]}\left(t_{1} \vee t_{2}\right)=0, \\
f_{3}\left(t_{1}, t_{2}, t_{3}\right) & =\frac{1}{6} \mathbb{E}\left(D_{t_{1}, t_{2}, t_{3}}^{3} B_{1}^{3}\right)=\mathbf{1}_{[0,1]}\left(t_{1} \vee t_{2} \vee t_{3}\right),
\end{aligned}
$$

and we obtain the Wiener chaos expansion

$$
B_{1}^{3}=3 B_{1}+6 \int_{0}^{1} \int_{0}^{t_{1}} \int_{0}^{t_{2}} d B_{t_{1}} d B_{t_{2}} d B_{t_{3}} .
$$

\subsubsection{Divergence on the Wiener chaos}

We now compute the divergence operator on the Wiener chaos expansion. A square integrable stochastic process $u \in L^{2}(\Omega \times[0, T])$ has an orthogonal expansion of the form

$$
u_{t}=\sum_{n=0}^{\infty} I_{n}\left(f_{n}(\cdot, t)\right),
$$

where $f_{0}(t)=\mathbb{E}\left(u_{t}\right)$ and, for each $n \geq 1, f_{n} \in L^{2}\left([0, T]^{n+1}\right)$ is a symmetric function in the first $n$ variables.

Proposition 1.3.4. The process $u$ belongs to the domain of $\delta$ if and only if the series

$$
\delta(u)=\sum_{n=0}^{\infty} I_{n+1}\left(\tilde{f}_{n}\right)
$$

converges in $L^{2}(\Omega)$.

Proof. Suppose that $G=I_{n}(g)$ is a multiple stochastic integral of order $n \geq 1$, where $g$ is symmetric. Then

$$
\begin{aligned}
\mathbb{E}\left(\langle u, D G\rangle_{\mathfrak{H}}\right) & =\int_{0}^{T} \mathbb{E}\left(I_{n-1}\left(f_{n-1}(\cdot, t)\right) n I_{n-1}(g(\cdot, t))\right) d t \\
& =n(n-1) ! \int_{0}^{T}\left\langle f_{n-1}(\cdot, t), g(\cdot, t)\right\rangle_{L^{2}\left([0, T]^{n-1}\right)} d t \\
& =n !\left\langle f_{n-1}, g\right\rangle_{L^{2}\left([0, T]^{n}\right)}=n !\left\langle\tilde{f}_{n-1}, g\right\rangle_{L^{2}\left([0, T]^{n}\right)} \\
& =\mathbb{E}\left(I_{n}\left(\tilde{f}_{n-1}\right) I_{n}(g)\right)=\mathbb{E}\left(I_{n}\left(\tilde{f}_{n-1}\right) G\right) .
\end{aligned}
$$

If $u \in \operatorname{Dom} \delta$, we deduce that

$$
\mathbb{E}(\delta(u) G)=\mathbb{E}\left(I_{n}\left(\tilde{f}_{n-1}\right) G\right)
$$

for every $G \in \mathcal{H}_{n}$. This implies that $I_{n}\left(\tilde{f}_{n-1}\right)$ coincides with the projection of $\delta(u)$ on the $n$th Wiener chaos. Consequently, the series in (1.22) converges in $L^{2}(\Omega)$ and its sum is equal to $\delta(u)$. The converse can be proved by similar arguments. 


\subsection{Criterion of differentiability}

Proposition 1.4.1. Let $\left(F_{n}\right)_{n \geq 1}$ be a sequence of random variables in $\mathbb{D}^{1, p}, p>1$, that converges to $F$ in $L^{p}(\Omega)$ and is such that

$$
\sup _{n} \mathbb{E}\left(\left\|D F_{n}\right\|_{\mathfrak{H}}^{p}\right)<\infty .
$$

Then $F$ belongs to $\mathbb{D}^{1, p}$ and the sequence of derivatives $\left(D F_{n}\right)_{n \geq 1}$ converges to $D F$ in the weak topology of $L^{p}(\Omega ; \mathfrak{H})$.

Proof. We will present here the proof in the case $p=2$, based on Wiener chaos expansions, the proof in the general case being more involved.

The assumptions imply that there exists a subsequence $\left(F_{n(k)}\right)_{k \geq 1}$ such that the sequence of derivatives $\left(D F_{n(k)}\right)_{k \geq 1}$ converges in the weak topology of $L^{2}(\Omega ; \mathfrak{H})$ to some element $\alpha \in L^{\overline{2}}(\Omega ; \mathfrak{H})$. By the duality formula, we have

$$
\begin{aligned}
\mathbb{E}\left(\langle\alpha, h\rangle_{\mathfrak{H}} G\right) & =\lim _{k \rightarrow \infty} \mathbb{E}\left(\left\langle D F_{n(k)}, h\right\rangle_{\mathfrak{H}} G\right) \\
& =\lim _{k \rightarrow \infty} \mathbb{E}\left(F_{n(k)} \delta(G h)\right) \\
& =\mathbb{E}(F \delta(G h)) .
\end{aligned}
$$

Let $F=\sum_{n=0}^{\infty} I_{n}\left(f_{n}\right)$. Then,

$$
\begin{aligned}
\sum_{n=1}^{\infty} \mathbb{E}\left(\left\langle n I_{n-1}\left(f_{n}(\cdot, t)\right), h\right\rangle_{\mathfrak{H}} G\right) & =\sum_{n=0}^{\infty} \mathbb{E}\left(\left\langle D\left(I_{n}\left(f_{n}\right)\right), h\right\rangle_{\mathfrak{H}} G\right) \\
& =\sum_{n=0}^{\infty} \mathbb{E}\left(I_{n}\left(f_{n}\right) \delta(G h)\right) \\
& =\mathbb{E}(F \delta(G h))=\mathbb{E}\left(\langle\alpha, h\rangle_{\mathfrak{H}} G\right),
\end{aligned}
$$

which implies that the series $\sum_{n=1}^{\infty} n I_{n-1}\left(f_{n}(\cdot, t)\right)$ converges in $L^{2}(\Omega ; \mathfrak{H})$ and its sum is $\alpha$. Therefore, $F \in \mathbb{D}^{1,2}$ and $D F=\alpha$. This completes the proof.

As a consequence we can prove the following chain rule for Lipschitz functions:

Proposition 1.4.2. Let $\varphi: \mathbb{R}^{m} \rightarrow \mathbb{R}$ by a function such that

$$
|\varphi(x)-\varphi(y)| \leq K|x-y|
$$

for any $x, y \in \mathbb{R}^{m}$. Suppose $F=\left(F^{1}, \ldots, F^{m}\right)$ is such that $F^{j} \in \mathbb{D}^{1,2}$. Then, $\varphi(F) \in \mathbb{D}^{1,2}$ and there exists a random vector $G=\left(G^{1}, \ldots, G^{m}\right)$ bounded by $K$, such that

$$
D[\varphi(F)]=\sum_{j=1}^{m} G^{j} D F^{j} .
$$


Proof. First, show the result when $\varphi \in C^{1}\left(\mathbb{R}^{m}\right)$. In this case, $G^{j}=$ $\partial_{j} \varphi(F)$. Then, we approximate $\varphi$ by $\varphi * \epsilon_{n}$, where $\epsilon_{n}$ is an approximation to the identity and use the criterion for differentiabiilty.

\subsection{Ornstein-Uhlenbeck semigroup}

In this section we describe the main properties of the Ornstein-Uhlenbeck semigroup and its generator. We then give the relationship between the Malliavin derivative, the divergence operator, and the Ornstein-Uhlenbeck semigroup generator.

\subsubsection{Mehler's formula}

Let $B=\left(B_{t}\right)_{t \in[0, T]}$ be a Brownian motion on a the Wiener space $(\Omega, \mathcal{F}, P)$. Let $F$ be a random variable in $L^{2}(\Omega)$ with the Wiener chaos decomposition $F=\sum_{n=0}^{\infty} I_{n}\left(f_{n}\right), f_{n} \in L_{s}^{2}\left([0, T]^{n}\right)$.

Definition 1.5.1. The Ornstein-Uhlenbeck semigroup is the oneparameter semigroup $\left(T_{t}\right)_{t \geq 0}$ of operators on $L^{2}(\Omega)$ defined by

$$
T_{t}(F)=\sum_{n=0}^{\infty} e^{-n t} I_{n}\left(f_{n}\right) .
$$

An alternative and useful expression for the Ornstein-Uhlenbeck semigroup is Mehler's formula:

Proposition 1.5.1. Let $B^{\prime}=\left(B_{t}^{\prime}\right)_{t \geq 0}$ be an independent copy of $B$. Then, for any $t \geq 0$ and $F \in L^{2}(\Omega)$, we have

$$
T_{t}(F)=\mathbb{E}^{\prime}\left(F\left(e^{-t} B+\sqrt{1-e^{-2 t}} B^{\prime}\right)\right),
$$

where $\mathbb{E}^{\prime}$ denotes the mathematical expectation with respect to $B^{\prime}$.

Proof. Both $T_{t}$ in Definition 1.5.1 and the right-hand side of (1.23) give rise to linear contraction operators on $L^{2}(\Omega)$. Thus, it suffices to show (1.23) for random variables of the form $F=\exp \left(\lambda B(h)-\frac{1}{2} \lambda^{2}\right)$, where $B(h)=\int_{0}^{T} h_{t} d B_{t}, h \in \mathfrak{H}$, is an element of norm one, and $\lambda \in \mathbb{R}$. We have, using formula (1.17),

$$
\begin{aligned}
& \mathbb{E}^{\prime}\left(\exp \left(e^{-t} \lambda B(h)+\sqrt{1-e^{-2 t}} \lambda B^{\prime}(h)-\frac{1}{2} \lambda^{2}\right)\right) \\
& \quad=\exp \left(e^{-t} \lambda B(h)-\frac{1}{2} e^{-2 t} \lambda^{2}\right)=\sum_{n=0}^{\infty} e^{-n t} \frac{\lambda^{n}}{n !} H_{n}(B(h))=T_{t} F,
\end{aligned}
$$


because

$$
F=\sum_{n=0}^{\infty} \frac{\lambda^{n}}{n !} H_{n}(B(h))
$$

and $H_{n}(B(h))=I_{n}\left(h^{\otimes n}\right)$ (see Proposition 1.3.1). This completes the proof.

Here are two important consequences of Mehler's formula:

1.) The operator $T_{t}$ is nonnegative, that is, $F \geq 0 \Rightarrow T_{t} F \geq 0$.

2.) The operator $T_{t}$ is a contraction in $L^{p}(\Omega)$ for any $p \geq 1$ :

$$
\left\|T_{t} F\right\|_{p} \leq\|F\|_{p}
$$

Indeed, using Jensen's inequality, it follows that, for any $p \geq 1$,

$$
\begin{aligned}
\mathbb{E}\left(\left|T_{t}(F)\right|^{p}\right) & =\mathbb{E}\left(\left|\mathbb{E}^{\prime}\left(F\left(e^{-t} B+\sqrt{1-e^{-2 t}} B^{\prime}\right)\right)\right|^{p}\right) \\
& \leq \mathbb{E}\left(\mathbb{E}^{\prime}\left(\left|F\left(e^{-t} B+\sqrt{1-e^{-2 t}} B^{\prime}\right)\right|^{p}\right)\right)=\mathbb{E}\left(|F|^{p}\right) .
\end{aligned}
$$

\subsubsection{Hypercontractivity}

The Ornstein-Uhlenbeck semigroup has the following hypercontractivity property.

Theorem 1.5.2. Let $F \in L^{p}(\Omega), p>1$, and $q(t)=e^{2 t}(p-1)+1>p$, $t>0$. Then

$$
\left\|T_{t} F\right\|_{q(t)} \leq\|F\|_{p} .
$$

As a consequence of the hypercontractivity property, for any $1<p<$ $q<\infty$ the norms $\|\cdot\|_{p}$ and $\|\cdot\|_{q}$ are equivalent on any Wiener chaos $\mathcal{H}_{n}$. In fact, putting $q=e^{2 t}(p-1)+1>p$ with $t>0$, we obtain, for every $F \in \mathcal{H}_{n}$,

$$
e^{-n t}\|F\|_{q}=\left\|T_{t} F\right\|_{q} \leq\|F\|_{p},
$$

which implies that

$$
\|F\|_{q} \leq\left(\frac{q-1}{p-1}\right)^{n / 2}\|F\|_{p} .
$$

Moreover, for any $n \geq 1$ and $1<p<\infty$, the orthogonal projection onto the $n$th Wiener chaos $J_{n}$ is bounded in $L^{p}(\Omega)$, and

$$
\left\|J_{n} F\right\|_{p} \leq \begin{cases}(p-1)^{n / 2}\|F\|_{p} & \text { if } p>2 \\ (p-1)^{-n / 2}\|F\|_{p} & \text { if } p<2 .\end{cases}
$$

In fact, suppose first that $p>2$ and let $t>0$ be such that $p-1=e^{2 t}$. Using the hypercontractivity property with exponents $p$ and 2 , we obtain

$$
\left\|J_{n} F\right\|_{p}=e^{n t}\left\|T_{t} J_{n} F\right\|_{p} \leq e^{n t}\left\|J_{n} F\right\|_{2} \leq e^{n t}\|F\|_{2} \leq e^{n t}\|F\|_{p} .
$$


If $p<2$, we have

$$
\left\|J_{n} F\right\|_{p}=\sup _{\|G\|_{q} \leq 1} \mathbb{E}\left(\left(J_{n} F\right) G\right) \leq\|F\|_{p} \sup _{\|G\|_{q} \leq 1}\left\|J_{n} G\right\|_{q} \leq e^{n t}\|F\|_{p},
$$

where $q$ is the conjugate of $p$, and $q-1=e^{2 t}$.

\subsubsection{Generator of the Ornstein-Uhlenbeck semigroup}

The infinitesimal generator of the Ornstein-Uhlenbeck semigroup in $L^{2}(\Omega)$ is the operator given by

$$
L F=\lim _{t \downarrow 0} \frac{T_{t} F-F}{t},
$$

and the domain of $L$ is the set of random variables $F \in L^{2}(\Omega)$ for which the above limit exists in $L^{2}(\Omega)$. It is easy to show that a random variable $F=\sum_{n=0}^{\infty} I_{n}\left(f_{n}\right), f_{n} \in L_{s}^{2}\left([0, T]^{n}\right)$, belongs to the domain of $L$ if and only if

$$
\sum_{n=1}^{\infty} n^{2}\left\|I_{n}\left(f_{n}\right)\right\|_{2}^{2}<\infty
$$

and, in this case, $L F=\sum_{n=1}^{\infty}-n I_{n}\left(f_{n}\right)$. Thus, Dom $L$ coincides with the space $\mathbb{D}^{2,2}$.

We also define the operator $L^{-1}$, which is the pseudo-inverse of $L$, as follows. For every $F \in L^{2}(\Omega)$, set

$$
L F=-\sum_{n=1}^{\infty} \frac{1}{n} I_{n}\left(f_{n}\right)
$$

Note that $L^{-1}$ is an operator with values in $\mathbb{D}^{2,2}$ and that $L L^{-1} F=$ $F-\mathbb{E}(F)$, for any $F \in L^{2}(\Omega)$, so $L^{-1}$ acts as the inverse of $L$ for centered random variables.

The next proposition explains the relationship between the operators $D$, $\delta$, and $L$.

Proposition 1.5.2. Let $F \in L^{2}(\Omega)$. Then, $F \in \operatorname{Dom} L$ if and only if $F \in \mathbb{D}^{1,2}$ and $D F \in \operatorname{Dom} \delta$ and, in this case, we have

$$
\delta D F=-L F .
$$

Proof. Let $F=\sum_{n=0}^{\infty} I_{n}\left(f_{n}\right)$. Suppose first that $F \in \mathbb{D}^{1,2}$ and $D F \in$ Dom $\delta$. Then, for any random variable $G=I_{m}\left(g_{m}\right)$, we have, using the duality relationship (Proposition 1.2.1),

$$
\mathbb{E}(G \delta D F)=\mathbb{E}\left(\langle D G, D F\rangle_{\mathfrak{H}}\right)=m m !\left\langle g_{m}, f_{m}\right\rangle_{L^{2}\left([0, T]^{m}\right)}=\mathbb{E}\left(G m I_{m}\left(f_{m}\right)\right) .
$$


Therefore, the projection of $\delta D F$ onto the $m$ th Wiener chaos is equal to $m I_{m}\left(f_{m}\right)$. This implies that the series $\sum_{n=1}^{\infty} n I_{n}\left(f_{n}\right)$ converges in $L^{2}(\Omega)$ and its sum is $\delta D F$. Therefore, $F \in \operatorname{Dom} L$ and $L F=-\delta D F$.

Conversely, suppose that $F \in \operatorname{Dom} L$. Clearly, $F \in \mathbb{D}^{1,2}$. Then, for any random variable $G \in \mathbb{D}^{1,2}$ with Wiener chaos expansion $G=\sum_{n=0}^{\infty} I_{n}\left(g_{n}\right)$, we have

$$
\mathbb{E}\left(\langle D G, D F\rangle_{\mathfrak{H}}\right)=\sum_{n=1}^{\infty} n n !\left\langle g_{n}, f_{n}\right\rangle_{L^{2}\left([0, T]^{n}\right)}=-\mathbb{E}(G L F) .
$$

As a consequence, $D F$ belongs to the domain of $\delta$ and $\delta D F=-L F$.

The next proposition shows that the operator $L$ behaves as a secondorder differential operator.

Proposition 1.5.3. Suppose that $F=\left(F^{1}, \ldots, F^{m}\right)$ is a random vector whose components belong to $\mathbb{D}^{2,4}$. Let $\varphi$ be a function in $C^{2}\left(\mathbb{R}^{m}\right)$ with bounded first and second partial derivatives. Then, $\varphi(F) \in \operatorname{Dom} L$ and

$$
L(\varphi(F))=\sum_{i, j=1}^{m} \frac{\partial^{2} \varphi}{\partial x_{i} \partial x_{j}}(F)\left\langle D F^{i}, D F^{j}\right\rangle_{\mathfrak{H}}+\sum_{i=1}^{m} \frac{\partial \varphi}{\partial x_{i}}(F) L F^{i}
$$

Proof. By the chain rule (see Proposition 1.2.4), $\varphi(F)$ belongs to $\mathbb{D}^{1,2}$ and

$$
D(\varphi(F))=\sum_{i=1}^{m} \frac{\partial \varphi}{\partial x_{i}}(F) D F^{i}
$$

Moreover, by Proposition 1.5.2, $\varphi(F)$ belongs to Dom $L$ and $L(\varphi(F))=$ $-\delta(D(\varphi(F)))$. Using the factorization property of the divergence operator yields the result.

In the finite-dimensional case $\left(\Omega=\mathbb{R}^{n}\right.$ equipped with the standard Gaussian law), $L=\Delta-x \cdot \nabla$ coincides with the generator of the OrnsteinUhlenbeck process $\left(X_{t}\right)_{t \geq 0}$ in $\mathbb{R}^{n}$, which is the solution to the stochastic differential equation

$$
d X_{t}=\sqrt{2} d B_{t}-X_{t} d t
$$

where $\left(B_{t}\right)_{t \geq 0}$ is an $n$-dimensional Brownian motion.

\subsubsection{Second integral representation}

Recall that $L$ is the generator of the Ornstein-Uhlenbeck semigroup.

Proposition 1.5.4. Let $F$ be in $\mathbb{D}^{1,2}$ with $\mathbb{E}(F)=0$. Then the process

$$
u=-D L^{-1} F
$$


belongs to $\operatorname{Dom} \delta$ and satisfies $F=\delta(u)$. Moreover $u \in L^{2}(\Omega ; \mathfrak{H})$ is unique among all square integrable processes with a chaos expansion

$$
u_{t}=\sum_{q=0}^{\infty} I_{q}\left(f_{q}(t)\right)
$$

such that $f_{q}\left(t, t_{1}, \ldots, t_{q}\right)$ is symmetric in all $q+1$ variables $t, t_{1}, \ldots, t_{q}$.

Proof. By Proposition 1.5.2,

$$
F=L L^{-1} F=-\delta\left(D L^{-1} F\right)
$$

Clearly, the process $u=-D L^{-1} F$ has a Wiener chaos expansion with functions symmetric in all their variables. To show uniqueness, let $v \in L^{2}(\Omega ; \mathfrak{H})$ with a chaos expansion $v_{t}=\sum_{q=0}^{\infty} I_{q}\left(g_{q}(t)\right)$, such that the function $g_{q}\left(t, t_{1}, \ldots, t_{q}\right)$ is symmetric in all $q+1$ variables $t, t_{1}, \ldots, t_{q}$ and such that $\delta(v)=F$. Then, there exists a random variable $G \in \mathbb{D}^{1,2}$ such that $D G=v$. Indeed, it suffices to take

$$
G=\sum_{q=0}^{\infty} \frac{1}{q+1} I_{q+1}\left(g_{q}\right)
$$

We claim that $G=-L^{-1} F$. This follows from $L G=-\delta D G=-\delta(v)=$ $-F$. The proof is now complete.

It is important to notice that, unlike the Clark-Ocone formula, which requires that the underlying process is a Brownian motion, the representation provided in Proposition 1.5.4 holds in the context of a general Gaussian isonormal process.

\subsection{Existence and regularity of densities}

Let $F=\left(F^{1}, \ldots, F^{m}\right)$ be a random vector such that $F^{i} \in \mathbb{D}^{1,2}$ for $i=1, \ldots, m$. We define the Malliavin matrix of $F$ as the random symmetric nonnegative definite matrix

$$
\gamma_{F}=\left(\left\langle D F^{i}, D F^{j}\right\rangle_{\mathfrak{H}}\right)_{1 \leq i, j \leq m}
$$

In the one-dimensional case, $\gamma_{F}=\|D F\|_{\mathfrak{H}}^{2}$. The following theorem is a a basic criterion for the existence of a density.

Theorem 1.6.1. If $\operatorname{det} \gamma_{F}>0$ a.s. then the law of $F$ is absolutely continuous with respect to the Lebesgue measure on $\mathbb{R}^{m}$. 
This theorem was proved by Bouleau and Hirsch using the co-area formula and techniques of geometric measure theory, and we omit the proof. As a consequence, the measure $\left(\operatorname{det} \gamma_{F} \times P\right) \circ F^{-1}$ is always absolutely continuous; that is,

$$
P\left(F \in B, \operatorname{det} \gamma_{F}>0\right)=0,
$$

for any Borel set $B \in \mathcal{B}\left(\mathbb{R}^{m}\right)$ of zero Lebesgue measure.

Definition 1.6.2. We say that a random vector $F=\left(F^{1}, \ldots, F^{m}\right)$ is nondegenerate if $F^{i} \in \mathbb{D}^{1,2}$ for $i=1, \ldots, m$ and

$$
\mathbb{E}\left(\left(\operatorname{det} \gamma_{F}\right)^{-p}\right)<\infty
$$

for all $p \geq 2$.

The following theorem is the basic criterion for the smoothness of densities.

Theorem 1.6.3. Let $F=\left(F^{1}, \ldots, F^{m}\right)$ be a nondegenerate random vector such that $F^{i} \in \mathbb{D}^{\infty}$ for all $i=1, \ldots, m$. Then the law of $F$ possesses an infinitely differentiable density.

As an example of application of the above criterion, let $F=X_{t}$, where $\left(X_{t}\right)_{t g e 0}$ is a diffusion process on $\mathbb{R}^{m}$

$$
d X_{t}=b\left(X_{t}\right) d t+\sum_{k=1}^{d} \sigma_{k}\left(X_{t}\right) d B_{t}^{k}, \quad X_{0}=x_{0}
$$

Theorem 1.6.4. Suppose that the coefficients $b, \sigma_{k}: \mathbb{R}^{m} \rightarrow \mathbb{R}^{m}$ are infinitely differentiable with bounded partial derivatives. Then, $X_{t}^{j} \in \mathbb{D}^{\infty}$ for all $t \geq 0$ and $j=1 \ldots, m$. If, in addition, the Lie algebra spanned by $\left\{\sigma_{1}, \ldots, \sigma_{d}\right\}$ at $x=x_{0}$ is $\mathbb{R}^{m}$, where $\sigma_{k}=\sum_{i=1}^{m} \sigma_{k}^{i} \frac{\partial}{\partial x_{i}}$, then for any $t>0$ $\left(\operatorname{det} \gamma_{X_{t}}\right)^{-1} \in \cap_{p \geq 2} L^{p}(\Omega)$ and, by Theorem 1.6.3, the density $p_{t}(x)$ of $X_{t}$ is $\mathcal{C}^{\infty}$.

Notice that $p_{t}(x)$ satisfies the Fokker-Planck equation

$$
\left(-\frac{\partial}{\partial t}+\mathcal{L}^{*}\right) p_{t}=0
$$

where

$$
\mathcal{L}=\frac{1}{2} \sum_{i, j=1}^{m}\left(\sigma \sigma^{T}\right)^{i j} \frac{\partial^{2}}{\partial x_{i} \partial x_{j}}+\sum_{i=1}^{m} b^{i} \frac{\partial}{\partial x_{i}} .
$$

Then, $p_{t} \in C^{\infty}$ means that $\frac{\partial}{\partial t}-\mathcal{L}^{*}$ is hypoelliptic (Hörmander's theorem). 


\section{Chapter 2}

\section{Malliavin-Stein's approach for normal approximations}

\subsection{Stein's method for normal approxima- tions}

The following lemma is a characterization of the standard normal distribution on the real line.

Lemma 2.1.1 (Stein's lemma). A random variable $Z$ such that $\mathbb{E}(|Z|)<\infty$ has the standard normal distribution $N(0,1)$ if and only if, for any function $f \in C_{b}^{1}(\mathbb{R})$, we have

$$
\mathbb{E}\left(f^{\prime}(Z)-f(Z) Z\right)=0
$$

Proof. Suppose first that $Z$ has the standard normal distribution $N(0,1)$. Then, equality (2.1) follows integrating by parts and using that the density $p(x)=(1 / \sqrt{2 \pi}) \exp \left(-x^{2} / 2\right)$ satisfies the differential equation

$$
p^{\prime}(x)=-x p(x)
$$

Conversely, let $\varphi(\lambda)=\mathbb{E}\left(e^{i \lambda Z}\right), \lambda \in \mathbb{R}$, be the characteristic function of $Z$. Because $Z$ is integrable, we know that $\varphi$ is differentiable and $\varphi^{\prime}(\lambda)=$ $i \mathbb{E}\left(Z e^{i \lambda Z}\right)$. By our assumption, this is equal to $-\lambda \varphi(\lambda)$. Therefore, $\varphi(\lambda)=\exp \left(-\lambda^{2} / 2\right)$, which concludes the proof.

If the expectation $\mathbb{E}\left(f^{\prime}(X)-f(X) X\right)$ is small for functions $f$ in some large set, we might conclude that the distribution of $X$ is close to the 
normal distribution. This is the main idea of Stein's method for normal approximations and the goal is to quantify this assertion in a proper way (see, for instance, [6]).

To implement this idea, consider a random variable $Z$ with the $N(0,1)$ distribution and fix a measurable function $h: \mathbb{R} \rightarrow \mathbb{R}$ such that $\mathbb{E}(|h(Z)|)<$ $\infty$. Stein's equation associated with $h$ is the linear differential equation

$$
f_{h}^{\prime}(x)-x f_{h}(x)=h(x)-\mathbb{E}(h(Z)), \quad x \in \mathbb{R} .
$$

Definition 2.1.2. A solution to equation (2.2) is an absolutely continuous function $f_{h}$ such that there exists a version of the derivative $f_{h}^{\prime}$ satisfying (2.2) for every $x \in \mathbb{R}$.

The next result provides the existence of a unique solution to Stein's equation.

Proposition 2.1.1. The function

$$
f_{h}(x)=e^{x^{2} / 2} \int_{-\infty}^{x}(h(y)-\mathbb{E}(h(Z))) e^{-y^{2} / 2} d y
$$

is the unique solution of Stein's equation (2.2) satisfying

$$
\lim _{x \rightarrow \pm \infty} e^{-x^{2} / 2} f_{h}(x)=0
$$

Proof. Equation (2.2) can be written as

$$
e^{x^{2} / 2} \frac{d}{d x}\left(e^{-x^{2} / 2} f_{h}(x)\right)=h(x)-\mathbb{E}(h(Z))
$$

This implies that any solution to equation (2.2) is of the form

$$
f_{h}(x)=c e^{x^{2} / 2}+e^{x^{2} / 2} \int_{-\infty}^{x}(h(y)-\mathbb{E}(h(Z))) e^{-y^{2} / 2} d y
$$

for some $c \in \mathbb{R}$. Taking into account that

$$
\lim _{x \rightarrow \pm \infty} \int_{-\infty}^{x}(h(y)-\mathbb{E}(h(Z))) e^{-y^{2} / 2} d y=0
$$

the asymptotic condition (2.4) is satisfied if and only if $c=0$.

Notice that, since $\int_{\mathbb{R}}(h(y)-\mathbb{E}(h(Z))) e^{-y^{2} / 2} d y=0$, we have

$$
\int_{-\infty}^{x}(h(y)-\mathbb{E}(h(Z))) e^{-y^{2} / 2} d y=-\int_{x}^{\infty}(h(y)-\mathbb{E}(h(Z))) e^{-y^{2} / 2} d y .
$$


Proposition 2.1.2. Let $h: \mathbb{R} \rightarrow[0,1]$ be a measurable function. Then the solution to Stein's equation $f_{h}$ given by (2.3) satisfies

$$
\left\|f_{h}\right\|_{\infty} \leq \sqrt{\frac{\pi}{2}} \quad \text { and } \quad\left\|f_{h}^{\prime}\right\|_{\infty} \leq 2 .
$$

Proof. Taking into account that $|h(x)-\mathbb{E}(h(Z))| \leq 1$, where $Z$ has law $N(0,1)$, we obtain

$$
\left|f_{h}(x)\right| \leq e^{x^{2} / 2} \int_{|x|}^{\infty} e^{-y^{2} / 2} d y=\sqrt{\frac{\pi}{2}},
$$

because the function $x \rightarrow e^{x^{2} / 2} \int_{|x|}^{\infty} e^{-y^{2} / 2} d y$ attains its maximum at $x=0$.

To prove the second estimate, observe that, in view of (2.5), we can write

$$
\begin{aligned}
f_{h}^{\prime}(x) & =h(x)-\mathbb{E}(h(Z))+x e^{x^{2} / 2} \int_{-\infty}^{x}(h(y)-\mathbb{E}(h(Z))) e^{-y^{2} / 2} d y \\
& =h(x)-\mathbb{E}(h(Z))-x e^{x^{2} / 2} \int_{x}^{\infty}(h(y)-\mathbb{E}(h(Z))) e^{-y^{2} / 2} d y
\end{aligned}
$$

for every $x \in \mathbb{R}$. Therefore

$$
\left|f_{h}^{\prime}(x)\right| \leq 1+|x| e^{x^{2} / 2} \int_{|x|}^{\infty} e^{-y^{2} / 2} d y=2 .
$$

This completes the proof.

\subsubsection{Total variation and convergence in law}

Let $F_{n}$ be a sequence of random variables defined in a probability space $(\Omega, \mathcal{F}, P)$.

Definition 2.1.3. We say that $F_{n} \stackrel{\mathcal{L}}{\rightarrow} F$ if $\mathbb{E}\left[g\left(F_{n}\right)\right] \rightarrow \mathbb{E}[g(F)]$ for any $g: \mathbb{R} \rightarrow \mathbb{R}$ continuous and bounded.

We know that $F_{n} \stackrel{\mathcal{L}}{\rightarrow} F$ if and only if $P\left(F_{n} \leq z\right) \rightarrow P(F \leq z)$ for any point $z \in \mathbb{R}$ of continuity of the distribution function of $F$.

The total variation distance between two probabilities $\nu_{1}$ and $\nu_{2}$ on $\mathbb{R}$ is defined as

$$
d_{T V}\left(\nu_{1}, \nu_{2}\right)=\sup _{B \in \mathcal{B}(\mathbb{R})}\left|\nu_{1}(B)-\nu_{2}(B)\right| .
$$

Then, the convergence $d_{T V}\left(P \circ F_{n}^{-1}, P \circ F^{-1}\right) \rightarrow 0$ is strictly stronger that the convergence in law $F_{n} \stackrel{\mathcal{L}}{\rightarrow} F$. By an abuse of notation we will write $d_{T V}(F, G)$ for $d_{T V}\left(P \circ F^{-1}, P \circ G^{-1}\right)$.

Using Stein's method, we can prove the following result. 
Proposition 2.1.3. Let $\nu$ be a probability on $\mathbb{R}$. Then,

$$
d_{T V}(\nu, \gamma) \leq \sup _{\phi \in \mathscr{F}_{T V}}\left|\int_{\mathbb{R}}\left[\phi^{\prime}(x)-x \phi(x)\right] \nu(d x)\right|,
$$

where

$$
\mathscr{F}_{T V}=\left\{\phi \in C^{1}(\mathbb{R}):\|\phi\|_{\infty} \leq \sqrt{\frac{\pi}{2}},\left\|\phi^{\prime}\right\|_{\infty} \leq 2\right\}
$$

and $\gamma$ denotes the standard normal distribution.

Proof. Let $h: \mathbb{R} \rightarrow[0,1]$ be a continuous function and let $\phi_{h}$ be the solution to the Stein's equation associated with $h$, that is,

$$
h(x)-E[h(Z)]=\phi_{h}^{\prime}(x)-x \phi_{h}(x) .
$$

Integrating with respect to $\nu$ yields

$$
\begin{aligned}
\left|\int_{\mathbb{R}} h d \nu-\int_{\mathbb{R}} h d \gamma\right| & =\left|\int_{\mathbb{R}}\left[\phi_{h}^{\prime}(x)-x \phi_{h}(x)\right] \nu(d x)\right| \\
& \leq \sup _{\phi \in C^{1}(\mathbb{R}):\|\phi\|_{\infty} \leq \sqrt{\frac{\pi}{2}},\left\|\phi^{\prime}\right\|_{\infty} \leq 2}\left|\int_{\mathbb{R}}\left[\phi^{\prime}(x)-x \phi(x)\right] \nu(d x)\right| .
\end{aligned}
$$

This inequality holds for any $h: \mathbb{R} \rightarrow[0,1]$ measurable, because we can approximate $h$ by continuous functions almost everywhere with respect to the measure $\nu+\gamma$. Taking $h=\mathbf{1}_{B}$, we obtain the result.

\subsection{Stein meets Malliavin}

Let $\left(B_{t}\right)_{\in[0, T]}$ be a Brownian motion defined on the Wiener space $(\Omega, \mathcal{F}, P)$. The following results connects Stein's method with Malliavin calculus.

Theorem 2.2.1. Suppose that $F \in \mathbb{D}^{1,2}$ satisfies $F=\delta(u)$, where $u$ belongs to the domain in $L^{2}$ of the divergence operator $\delta$. Then,

$$
d_{T V}(F, Z) \leq 2 \mathbb{E}\left[\left|1-\langle D F, u\rangle_{\mathfrak{H}}\right|\right],
$$

where $Z$ is a $N(0,1)$ random variable.

Proof. Using the duality relationship between the operators $D$ and $\delta$ and chain rule, we can write

$$
\mathbb{E}[F \phi(F)]=\mathbb{E}[\delta(u) \phi(F)]=\mathbb{E}\left[\langle u, D[\phi(F)]\rangle_{\mathfrak{H}}\right]=\mathbb{E}\left[\phi^{\prime}(F)\langle u, D F\rangle_{\mathfrak{H}}\right] .
$$

Therefore,

$$
\begin{aligned}
\left|\mathbb{E}\left[\phi^{\prime}(F)\right]-\mathbb{E}(F \phi(F)]\right| & =\mid \mathbb{E}\left[\phi^{\prime}(F)\left[1-\langle D F, u\rangle_{\mathfrak{H}}\right] \mid\right. \\
& \leq 2 \mathbb{E}\left[\left|1-\langle D F, u\rangle_{\mathfrak{H}}\right|\right]
\end{aligned}
$$

for any $\phi \in \mathscr{F}_{T V}$. This concludes the proof in view of Proposition 2.1.3. 
Consider the following example. Suppose that $F=\int_{0}^{T} u_{s} d B_{s}$, where $u$ is an adapted measurable process in $\mathbb{D}^{1,2}(\mathfrak{H})$. Then,

$$
D_{t} F=u_{t}+\int_{t}^{T} D_{t} u_{s} d B_{s}
$$

and

$$
\langle u, D F\rangle_{\mathfrak{H}}=\|u\|_{\mathfrak{H}}^{2}+\int_{0}^{T}\left(\int_{t}^{T} D_{t} u_{s} d B_{s}\right) u_{t} d t .
$$

As a consequence,

$$
\begin{aligned}
d_{T V}(F, Z) & \leq 2 \mathbb{E}\left(\left|1-\|u\|_{\mathfrak{H}}^{2}\right|\right)+2 \mathbb{E}\left(\left|\int_{0}^{T}\left(\int_{t}^{T} D_{t} u_{s} d B_{s}\right) u_{t} d t\right|\right) \\
& \leq 2 \mathbb{E}\left(\left|1-\|u\|_{\mathfrak{H}}^{2}\right|\right)+2\left[\mathbb{E} \int_{0}^{T}\left(\int_{0}^{s} u_{t} D_{t} u_{s} d t\right)^{2} d s\right]^{\frac{1}{2}} .
\end{aligned}
$$

These computations lead to the following result.

Proposition 2.2.1. A sequence $F_{n}=\int_{0}^{T} u_{s}^{(n)} d B_{s}$, where $u^{(n)}$ is an adapted and measurable process such that $u^{(n)} \in \mathbb{D}^{1,2}(\mathfrak{H})$, converges in total variation to the law $N(0,1)$ if:

(i) $\left\|u^{(n)}\right\|_{\mathfrak{H}}^{2} \rightarrow 1$ in $L^{1}(\Omega)$ and

(ii) $\mathbb{E} \int_{0}^{T}\left(\int_{0}^{s} u_{t}^{(n)} D_{t} u_{s}^{(n)} d t\right)^{2} d s \rightarrow 0$.

Example: The previous proposition can be applied to the following process:

$$
u_{t}^{(n)}=\sqrt{2 n} t^{n} \exp \left(B_{t}(1-t)\right) \mathbf{1}_{[0,1]}(t) .
$$

In Theorem 2.2.1 we can take $u=-D L^{-1} F$, because

$$
F=L L^{-1} F=-\delta D L^{-1} F
$$

and, we obtain

$$
d_{T V}(F, Z) \leq 2 \mathbb{E}\left[\left|1-\left\langle D F,-D L^{-1} F\right\rangle_{\mathfrak{H}}\right|\right]
$$

Suppose that $\mathbb{E}\left[F^{2}\right]=\sigma^{2}>0$. Then, we can derive the following inequality:

$$
d_{T V}(F, \sigma Z) \leq \frac{2}{\sigma^{2}} \mathbb{E}\left[\left|\sigma^{2}-\langle D F, u\rangle_{\mathfrak{H}}\right|\right]
$$


Indeed,

$$
\begin{aligned}
d_{T V}(F, \sigma Z) & =\sup _{B \in \mathcal{B}(\mathbb{R})}|P(F \in B)-P(\sigma Z \in B)| \\
& =\sup _{B \in \mathcal{B}(\mathbb{R})}\left|P\left(\sigma^{-1} F \in \sigma^{-1} B\right)-P\left(Z \in \sigma^{-1} B\right)\right| \\
& \leq \frac{2}{\sigma^{2}} \mathbb{E}\left[\left|\sigma^{2}-\langle D F, u\rangle_{\mathfrak{H}}\right|\right] .
\end{aligned}
$$

Moreover,

$$
\mathbb{E}\left[\langle D F, u\rangle_{\mathfrak{H}}\right]=\mathbb{E}[\delta(u) F]=\mathbb{E}\left[F^{2}\right]=\sigma^{2},
$$

and we obtain

$$
d_{T V}(F, \sigma Z) \leq \frac{2}{\sigma^{2}} \sqrt{\operatorname{Var}\left(\langle D F, u\rangle_{\mathfrak{H}}\right)}
$$

\subsubsection{Normal approximation on a fixed Wiener chaos}

For random variables on a fixed Wiener chaos we can prove the following result.

Proposition 2.2.2. Suppose $F \in \mathcal{H}_{q}$ for some $q \geq 2$ and $\mathbb{E}\left(F^{2}\right)=\sigma^{2}$. Then,

$$
d_{T V}(F, \sigma Z) \leq \frac{2}{q \sigma^{2}} \sqrt{\operatorname{Var}\left(\|D F\|_{\mathfrak{H}}^{2}\right)} .
$$

Proof. Suppose that $F=I_{q}(f)$, where $f \in L_{s}^{2}\left([0, T]^{q}\right)$. We have

$$
D_{t} F=q I_{q-1}(f(\cdot, t))
$$

and $F=\delta(u)$, where

$$
u_{t}=I_{q-1}(f(\cdot, t))=\frac{1}{q} D_{t} F
$$

Therefore,

$$
\langle D F, u\rangle_{\mathfrak{H}}=\frac{1}{q}\|D F\|_{\mathfrak{H}}^{2}
$$

and it suffices to apply (2.7).

The next result states the equivalence between the variance of $\|D F\|_{\mathfrak{H}}^{2}$ and the default in the moment of order 4 .

Proposition 2.2.3. Suppose that $F=I_{q}(f) \in \mathcal{H}_{q}, q \geq 2$. Then,

$$
\operatorname{Var}\left(\|D F\|_{\mathfrak{H}}^{2}\right) \leq \frac{(q-1) q}{3}\left(\mathbb{E}\left(F^{4}\right)-3 \sigma^{4}\right) \leq(q-1) \operatorname{Var}\left(\|D F\|_{\mathfrak{H}}^{2}\right)
$$


Proof. This proposition is a consequence of the following two formulas. The first formula is the next one:

$$
\operatorname{Var}\left(\|D F\|_{\mathfrak{H}}^{2}\right)=\sum_{r=1}^{q-1} r^{2}(r !)^{2}\left(\begin{array}{c}
q \\
r
\end{array}\right)^{4}(2 q-2 r) !\left\|f \widetilde{\otimes}_{r} f\right\|_{\mathfrak{H}^{\otimes(2 q-2 r)}}^{2} .
$$

Proof of (2.8): We have $D_{t} F=q I_{q-1}(f(\cdot, t))$, and using the product formula for multiple stochastic integrals we obtain

$$
\begin{aligned}
\|D F\|_{\mathfrak{H}}^{2} & =q^{2} \int_{0}^{T} I_{q-1}(f(\cdot, t))^{2} d t \\
& =q^{2} \sum_{r=0}^{q-1} r !\left(\begin{array}{c}
q-1 \\
r
\end{array}\right)^{2} I_{2 q-2 r-2}\left(f \widetilde{\otimes}_{r+1} f\right) \\
& =q^{2} \sum_{r=1}^{q}(r-1) !\left(\begin{array}{c}
q-1 \\
r-1
\end{array}\right)^{2} I_{2 q-2 r}\left(f \widetilde{\otimes}_{r} f\right) \\
& =q q !\|f\|_{\mathfrak{H} \otimes q}^{2}+q^{2} \sum_{r=1}^{q-1}(r-1) !\left(\begin{array}{c}
q-1 \\
r-1
\end{array}\right)^{2} I_{2 q-2 r}\left(f \widetilde{\otimes}_{r} f\right) .
\end{aligned}
$$

Then, (2.8) follows from the isometry property of multiple integrals.

The second formula is the following one:

$$
\mathbb{E}\left[F^{4}\right]-3 \sigma^{4}=\frac{3}{q} \sum_{r=1}^{q-1} r(r !)^{2}\left(\begin{array}{c}
q \\
r
\end{array}\right)^{4}(2 q-2 r) !\left\|f \widetilde{\otimes}_{r} f\right\|_{\mathfrak{H}^{\otimes(2 q-2 r)}}^{2}
$$

Proof on (2.10): Using that $-L^{-1} F=\frac{1}{q} F$ and $L=-\delta D$ we can write

$$
\begin{aligned}
\mathbb{E}\left[F^{4}\right] & =\mathbb{E}\left[F \times F^{3}\right]=\mathbb{E}\left[\left(-\delta D L^{-1} F\right) F^{3}\right]=\mathbb{E}\left[\left\langle-D L^{-1} F, D\left(F^{3}\right)\right\rangle_{\mathfrak{H}}\right] \\
& =\frac{1}{q} \mathbb{E}\left[\left\langle D F, D\left(F^{3}\right)\right\rangle_{\mathfrak{H}}\right]=\frac{3}{q} \mathbb{E}\left[F^{2}\|D F\|_{\mathfrak{H}}^{2}\right]
\end{aligned}
$$

By the product formula of multiple integrals,

$$
F^{2}=I_{q}(f)^{2}=q !\|f\|_{\mathfrak{H}^{\otimes q}}^{2}+\sum_{r=0}^{q-1} r !\left(\begin{array}{c}
q \\
r
\end{array}\right)^{2} I_{2 q-2 r}\left(f \widetilde{\otimes}_{r} f\right)
$$

Then (2.10) follows from (2.11), (2.12), (2.9) and the isometry property of multiple integrals.

\subsubsection{Fourth Moment theorem}

Stein's method combined with Malliavin calculus leads to a simple proof of the Fourth Moment theorem: 
Theorem 2.2.2 (Nualart and Peccati [22], Nualart and Ortiz-Latorre [23]). Fix $q \geq 2$. Let $F_{n}=I_{q}\left(f_{n}\right) \in \mathcal{H}_{q}, n \geq 1$ be such that

$$
\lim _{n \rightarrow \infty} \mathbb{E}\left(F_{n}^{2}\right)=\sigma^{2}
$$

The following conditions are equivalent:

(i) $F_{n} \stackrel{\mathcal{L}}{\rightarrow} N\left(0, \sigma^{2}\right)$, as $n \rightarrow \infty$.

(ii) $\mathbb{E}\left(F_{n}^{4}\right) \rightarrow 3 \sigma^{4}$, as $n \rightarrow \infty$.

(iii) $\left\|D F_{n}\right\|_{\mathfrak{H}}^{2} \rightarrow q \sigma^{2}$ in $L^{2}(\Omega)$, as $n \rightarrow \infty$.

(iv) For all $1 \leq r \leq q-1, f_{n} \otimes_{r} f_{n} \rightarrow 0$, as $n \rightarrow \infty$.

This theorem constitutes a drastic simplification of the method of moments.

Proof. First notice that (i) implies (ii) because for any $p>2$, the hypercontractivity property of the Ornstein-Uhlenbeck semigroup (see (1.24)) implies

$$
\sup _{n}\left\|F_{n}\right\|_{p} \leq(p-1)^{\frac{q}{2}} \sup _{n}\left\|F_{n}\right\|_{2}<\infty
$$

The equivalence of (ii) and (iii)) follows from the previous proposition, and these conditions imply (i), with convergence in total variation. The fact that (iv) implies (ii) and (iii) is a consequence of $\left\|f_{n} \widetilde{\otimes}_{r} f_{n}\right\|_{\mathfrak{H} \otimes(2 q-2 r)} \leq$ $\left\|f_{n} \otimes_{r} f_{n}\right\|_{\mathfrak{H}^{\otimes(2 q-2 r)}}$. Let us show that (ii) implies (iv). From (2.12) we get

$$
\begin{aligned}
\mathbb{E}\left[F_{n}^{4}\right]= & \sum_{r=0}^{q}(r !)^{2}\left(\begin{array}{c}
q \\
r
\end{array}\right)^{4}(2 q-2 r) !\left\|f_{n} \tilde{\otimes}_{r} f_{n}\right\|_{\mathfrak{H} \otimes(2 q-2 r)}^{2} \\
= & (2 q) !\left\|f_{n} \tilde{\otimes} f_{n}\right\|_{\mathfrak{H}^{\otimes 2 q}}^{2}+\sum_{r=1}^{q-1}(r !)^{2}\left(\begin{array}{c}
q \\
r
\end{array}\right)^{4}(2 q-2 r) !\left\|f_{n} \tilde{\otimes}_{r} f_{n}\right\|_{\mathfrak{H} \otimes(2 q-2 r)}^{2} \\
& +(q !)^{2}\left\|f_{n}\right\|_{\mathfrak{H}}^{4} .
\end{aligned}
$$

Then, we use the fact that $(2 q) !\left\|f_{n} \widetilde{\otimes} f_{n}\right\|_{\mathfrak{H}^{\otimes 2 q}}^{2}$ equals to $2(q !)^{2}\left\|f_{n}\right\|_{\mathfrak{H}}^{4}$ plus a linear combination of the terms $\left\|f_{n} \otimes_{r} f_{n}\right\|_{\mathfrak{H}^{\otimes(2 q-2 r)}}^{2}$, with $1 \leq r \leq q-1$, to conclude that

$$
\left\|f_{n} \otimes_{r} f_{n}\right\|_{\mathfrak{H}^{\otimes(2 q-2 r)}} \rightarrow 0, \quad 1 \leq r \leq q-1
$$




\subsubsection{Multivariate Gaussian approximation}

The next result is as multivariate extension of the fourth moment theorem.

Theorem 2.2.3 (Peccati and Tudor [25]). Let $d \geq 2$ and $1 \leq q_{1}<$ $\cdots<q_{d}$. Consider random vectors

$$
F_{n}=\left(F_{n}^{1}, \ldots, F_{n}^{d}\right)=\left(I_{q_{1}}\left(f_{n}^{1}\right), \ldots, I_{q_{d}}\left(f_{n}^{d}\right)\right),
$$

where $f_{n}^{i} \in L_{s}^{2}\left([0, T]^{q_{i}}\right)$. Suppose that, for any $1 \leq i \leq d$,

$$
\lim _{n \rightarrow \infty} \mathbb{E}\left[\left(F_{n}^{i}\right)^{2}\right]=\sigma_{i}^{2} .
$$

Then, the following two conditions are equivalent:

(i) $F_{n} \stackrel{\mathcal{L}}{\rightarrow} N_{d}(0, \Sigma)$, where $\Sigma$ is a diagonal matrix such that $\Sigma_{i i}=\sigma_{i}^{2}$.

(ii) For every $i=1, \ldots, d, F_{n}^{i} \stackrel{\mathcal{L}}{\rightarrow} N\left(0, \sigma_{i}^{2}\right)$.

Note that the convergence of the marginal distributions implies the joint convergence to a random vector with independent components.

\subsubsection{Chaotic Central Limit Theorem}

For general random variables, we can show easily the following chaotic central limit theorem.

Theorem 2.2.4 (Hu and Nualart [9]). Let $F_{n}=\sum_{q=1}^{\infty} I_{q}\left(f_{q, n}\right), n \geq 1$. Suppose that:

(i) For all $q \geq 1, q$ ! $\left\|f_{q, n}\right\|_{L^{2}\left([0, T]^{q}\right)}^{2} \rightarrow \sigma_{q}^{2}$ as $n \rightarrow \infty$.

(ii) For all $q \geq 2$ and $1 \leq r \leq q-1, f_{q, n} \otimes_{r} f_{q, n} \rightarrow 0$ as $n \rightarrow \infty$.

(iii) $q !\left\|f_{q, n}\right\|^{2} \leq \delta_{q}$, where $\sum_{q} \delta_{q}<\infty$.

Then, as $n$ tends to infinity

$$
F_{n} \stackrel{\mathcal{L}}{\rightarrow} N\left(0, \sigma^{2}\right), \quad \text { where } \quad \sigma^{2}=\sum_{q=1}^{\infty} \sigma_{q}^{2} .
$$

Assuming (i), condition (ii) is equivalent to (ii)' $: \lim _{n \rightarrow \infty} \mathbb{E}\left(I_{q}\left(f_{q, n}\right)^{4}\right)=$ $3 \sigma_{q}^{4}, q \geq 2$. This theorem implies the convergence in law of the whole sequence $\left(I_{q}\left(f_{q, n}\right), q \geq 1\right)$ to an infinite dimensional Gaussian vector with independent components. 


\section{Chapter 3}

\section{Central limit theorems for stationary sequences}

Suppose that $\left(X_{n}\right)_{n \geq 1}$ is a sequence of random variables, defined in some probability space $(\Omega, \mathcal{F}, P)$, which are independent and identically distributed, with zero mean and finite variance $\mathbb{E}\left[X_{1}^{2}\right]=\sigma^{2}$. Then, the classical central limit theorem says that

$$
\frac{1}{\sqrt{n}} \sum_{k=1}^{n} X_{k} \stackrel{\mathcal{L}}{\rightarrow} N\left(0, \sigma^{2}\right) .
$$

That means, for any $a \leq b$,

$$
\lim _{n \rightarrow \infty} P\left(a \leq \frac{1}{\sqrt{n}} \sum_{k=1}^{n} X_{k} \leq b\right)=\int_{a}^{b} \frac{1}{\sqrt{2 \pi \sigma^{2}}} e^{-x^{2} / 2 \sigma^{2}} d x .
$$

In this section we will apply the Malliavin-Stein's method to establish quantitative versions of the central limit theorem, where the independence property is replaced by stationarity and we consider random variables which are functionals of a given Gaussian process.

\subsection{Breuer-Major theorem}

Recall that $\gamma$ denotes the standard normal distribution and $H_{q}$ is the $q$-th Hermite polynomial. A function $g \in L^{2}(\mathbb{R}, \gamma)$ has Hermite rank $d \geq 1$ if

$$
g(x)=\sum_{q=d}^{\infty} c_{q} H_{q}(x), \quad c_{d} \neq 0 .
$$

For example, $g(x)=|x|^{p}-\int_{\mathbb{R}}|x|^{p} d \gamma(x), p \geq 1$, has Hermite rank 2 . 
Let $\xi=\left(\xi_{k}\right)_{k \in \mathbb{Z}}$ be a centered stationary Gaussian sequence with unit variance. Set $\rho(v)=\mathbb{E}\left[\xi_{0} \xi_{v}\right]$ for $v \in \mathbb{Z}$.

Theorem 3.1.1 (Breuer and Major [4]). Let $g \in L^{2}(\mathbb{R}, \gamma)$ with Hermite rank $d \geq 1$ and assume

$$
\sum_{k \in \mathbb{Z}}|\rho(k)|^{d}<\infty .
$$

Then,

$$
F_{n}:=\frac{1}{\sqrt{n}} \sum_{k=1}^{n} g\left(\xi_{k}\right) \stackrel{\mathcal{L}}{\rightarrow} N\left(0, \sigma^{2}\right),
$$

as $n \rightarrow \infty$, where

$$
\sigma^{2}=\sum_{m=d}^{\infty} m ! c_{m}^{2} \sum_{k \in \mathbb{Z}} \rho(k)^{m} .
$$

Note that $|\rho(k)| \leq 1$, so

$$
\sigma^{2} \leq \sum_{m=d}^{\infty} c_{m}^{2} m ! \sum_{k \in \mathbb{Z}}|\rho(k)|^{d}=\|g\|_{L^{2}(\mathbb{R}, \gamma)}^{2} \sum_{k \in \mathbb{Z}}|\rho(k)|^{d}<\infty .
$$

Proof. From the chaotic Central Limit Theorem, it suffices to consider the case $g=c_{q} H_{q}, q \geq d$. There exists a sequence $\left(e_{k}\right)_{k \geq 1}$ in $\mathfrak{H}=L^{2}([0, T])$ such that for each $j, k \geq 1$,

$$
\left\langle e_{k}, e_{j}\right\rangle_{\mathfrak{H}}=\rho(k-j) .
$$

The sequence $\left(B\left(e_{k}\right)\right)_{k>1}$ has the same law as $\left(\xi_{k}\right)_{k>1}$, and we may replace $F_{n}=\frac{c_{q}}{\sqrt{n}} \sum_{k=1}^{n} H_{q}\left(\xi_{k}\right)$ by

$$
G_{n}=\frac{c_{q}}{\sqrt{n}} \sum_{k=1}^{n} H_{q}\left(B\left(e_{k}\right)\right)=I_{q}\left(f_{q, n}\right),
$$

where $f_{q, n}=\frac{c_{q}}{\sqrt{n}} \sum_{k=1}^{n} e_{k}^{\otimes q}$. We can write

$$
q !\left\|f_{q, n}\right\|_{\mathfrak{H} \otimes q}^{2}=\frac{q ! c_{q}^{2}}{n} \sum_{k, j=1}^{n} \rho(k-j)^{q}=q ! c_{q}^{2} \sum_{v \in \mathbb{Z}} \rho(v)^{q}\left(1-\frac{|v|}{n}\right) \mathbf{1}_{\{|v|<n\}},
$$

and by the dominated convergence theorem

$$
\mathbb{E}\left[G_{n}^{2}\right]=q !\left\|f_{q, n}\right\|_{\mathfrak{H}^{\otimes q}}^{2} \rightarrow q ! c_{q}^{2} \sum_{v \in \mathbb{Z}} \rho(v)^{q}=\sigma^{2} .
$$


Applying the Fourth Moment Theorem, it suffices to show that for $r=$ $1, \ldots, q-1$,

$$
f_{q, n} \otimes_{r} f_{q, n}=\frac{c_{q}^{2}}{n} \sum_{k, j=1}^{n} \rho(k-j)^{r} e_{k}^{\otimes(q-r)} \otimes e_{j}^{\otimes(q-r)} \rightarrow 0
$$

We have

$\left\|f_{q, n} \otimes_{r} f_{q, n}\right\|_{\mathfrak{H}^{\otimes(2 q-2 r)}}^{2}=\frac{c_{q}^{4}}{n^{2}} \sum_{i, j, k, \ell=1}^{n} \rho(k-j)^{r} \rho(i-\ell)^{r} \rho(k-i)^{q-r} \rho(j-\ell)^{q-r}$.

Using $\left|\rho(k-j)^{r} \rho(k-i)^{q-r}\right| \leq|\rho(k-j)|^{q}+|\rho(k-i)|^{q}$, we obtain

$$
\begin{aligned}
\left\|f_{q, n} \otimes_{r} f_{q, n}\right\|_{\mathfrak{H} \otimes(2 q-2 r)}^{2} \leq & 2 c_{q}^{4} \sum_{k \in \mathbb{Z}}|\rho(k)|^{q}\left(n^{-1+\frac{r}{q}} \sum_{|i| \leq n}|\rho(i)|^{r}\right) \\
& \times\left(n^{-1+\frac{q-r}{q}} \sum_{|j| \leq n}|\rho(j)|^{q-r}\right) .
\end{aligned}
$$

Then, it suffices to show that for $r=1, \ldots, q-1$,

$$
n^{-1+\frac{r}{q}} \sum_{|i| \leq n}|\rho(i)|^{r} \rightarrow 0 \text {. }
$$

This follows from Hölder's inequality. Indeed, for a fixed $\delta \in(0,1)$, we have the estimates

$$
n^{-1+\frac{r}{q}} \sum_{|i| \leq[n \delta]}|\rho(i)|^{r} \leq n^{-1+\frac{r}{q}}(2[n \delta]+1)^{1-\frac{r}{q}}\left(\sum_{i \in \mathbb{Z}}|\rho(i)|^{q}\right)^{\frac{r}{q}} \leq c \delta^{1-\frac{r}{q}},
$$

and

$$
n^{-1+\frac{r}{q}} \sum_{[n \delta]<|i| \leq n}|\rho(i)|^{r} \leq\left(\sum_{[n \delta]<|i| \leq n}|\rho(i)|^{q}\right)^{\frac{r}{q}}
$$

The first term converges to zero as $\delta$ tends to zero and the second one converges to zero for fixed $\delta$ as $n \rightarrow \infty$.

\subsubsection{Convergence in law in $C([0, T])$}

In the framework of the Breuer-Major theorem, we are interested in the convergence in law in the space $C([0, T])$. 
Definition 3.1.2. Let $Y_{n}:=\left(Y_{n}(t)\right)_{t \in[0, T]}$ be a sequence of continuous stochastic processes. We say that this sequence converges in law to a continuous process $Y$ if for any continuous and bounded function $\varphi$ : $C([0, T]) \rightarrow C([0, T])$, we have

$$
\lim _{n \rightarrow \infty} \mathbb{E}\left(\varphi\left(Y_{n}\right)\right)=\mathbb{E}(\varphi(Y))
$$

A basic ingredient to show convergence in law in an infinite dimensional space is the notion of tightness.

Definition 3.1.3. A sequence of probabilities $\left(\nu_{n}\right)_{n \geq 1}$ on $C([0, T])$ is tight if for any $\epsilon>0$ there exists a compact set $K_{\epsilon} \subset C([0, T])$ such that

$$
\sup _{n \geq 1} \nu_{n}\left(K_{\epsilon}^{c}\right) \leq \epsilon
$$

By the Arzelà-Ascoli theorem, a set of functions $K \subset C([0, T])$ is compact if and only if :

(i) $\sup _{x \in K, t \in[0, T]}|x(t)|<\infty$.

(ii) $K$ is an equicontinuous set: For all $\epsilon>0$ there exists $\delta>0$ such that for all $x \in K$,

$$
|s-t|<\delta \Rightarrow|x(s)-x(t)| \leq \epsilon
$$

This leads to the folliwing criterion for tightness on $C([0, T])$ :

Proposition 3.1.1. Consider a sequence of continuous stochastic processes $Y_{n}=\left(Y_{n}(t)\right)_{t \in[0, T]}$ defined in a probability space $(\Omega, \mathcal{F}, P)$ and set

$$
\nu_{n}(B):=P\left(Y_{n}^{-1}(B)\right),
$$

for any Borel set in $C([0, T])$. The sequence $\nu_{n}=P \circ Y_{n}^{-1}$ is tight if

(i) $\sup _{n \geq 1} P\left(\left|Y_{n}(0)\right|>a\right) \rightarrow 0$ as $a \uparrow \infty$.

(ii) For some $\alpha>1$ and $p \geq 1$.

$$
\mathbb{E}\left[\left|Y_{n}(t)-Y_{n}(s)\right|^{p}\right] \leq c|t-s|^{\alpha},
$$

It is well known that proving convergence in law in $C([0, T])$ requires showing convergence of the finite-dimensional distributions and proving tightness. This is the contents of the next theorem.

Theorem 3.1.4. Let $Y_{n}=\left(Y_{n}(t)\right)_{t \in[0, T]}$ be a sequence of continuous stochastic processes. Then $Y_{n}$ converges in law to a continuous process $Y$ if: 
(i) The finite-dimensional distributions of $Y_{n}$ converge in law to those of $Y$. That is, for any $0 \leq t_{1}<\cdots \leq t_{M} \leq T$, we have, as $n \rightarrow \infty$,

$$
\left(Y_{n}\left(t_{1}\right), \ldots, Y_{n}\left(t_{M}\right)\right) \stackrel{\mathcal{L}}{\rightarrow}\left(Y\left(t_{1}\right), \ldots, Y\left(t_{M}\right)\right)
$$

(ii) The sequence of laws $\nu_{n}=P \circ Y_{n}^{-1}$ is tight.

In view of Proposition 3.1.1, a sufficient condition for (ii) is: $\sup _{n \geq 1} \mathbb{E}\left(\left|Y_{n}(0)\right|^{p}\right)<\infty$ and

$$
\mathbb{E}\left[\left|Y_{n}(t)-Y_{n}(s)\right|^{p}\right] \leq c|t-s|^{\alpha},
$$

for some $\alpha>1$ and $p \geq 1$,

\subsubsection{Functional version of the Breuer-Major theorem}

In the framework of the Breuer-Major theorem, define $Y_{n}$ as the continuous process on $[0, T]$ obtained by linear interpolation from

$$
Y_{n}(N / n)=\frac{1}{\sqrt{n}} \sum_{k=1}^{N} g\left(\xi_{k}\right), \quad N=1,2, \ldots
$$

The following result is a functional version of the Breuer-Major theorem.

Theorem 3.1.5 (Nourdin and Nualart [14]). Let $\xi=\left(\xi_{n}\right)_{n \in \mathbb{Z}}$ be a centered Gaussian stationary sequence with unit variance and covariance $\rho$. Let $g \in L^{2}(\mathbb{R}, \gamma)$ with Hermite rank $d \geq 1$. Suppose that:

(i) $\sum_{k \in \mathbb{Z}}|\rho(k)|^{d}<\infty$.

(ii) $g \in L^{p}(\mathbb{R}, \gamma)$ for some $p>2$.

Then, as $n \rightarrow \infty$,

$$
\left(Y_{n}(t)\right)_{t \in[0, T]} \stackrel{\mathcal{L}}{\rightarrow} \sigma B,
$$

where $B$ is a Brownian motion, the convergence holds in law in $C([0, T])$ and $\sigma$ is defined in (3.1).

When the random variables $\xi_{n}$ are independent, this is the classical Donsker theorem: The random walk converges in distribution to the Brownian motion.

Remark: Chambers and Slud [5] and Ben-Hariz [1] proved the functional version of the Breuer-Major theorem under the following condition on the rate of the convergence of the coefficients:

(ii') $m ! c_{m}^{2} \leq C \alpha^{m}, \quad m \geq d$ 
for some $C>0$ and $\alpha<1$.

By the hypercontractivity property, (ii') implies that $g \in L^{p}(\mathbb{R}, \gamma)$ for $2<p<\frac{1}{\alpha}+1$. In fact,

$$
\begin{aligned}
\|g\|_{L^{p}(\mathbb{R}, \gamma)} & \leq \sum_{m=d}^{\infty}\left|c_{m}\right|\left\|H_{m}\right\|_{L^{p}(\mathbb{R}, \gamma)} \\
& \leq \sum_{m=d}^{\infty}\left|c_{m}\right|(p-1)^{\frac{m}{2}} \sqrt{m !} \\
& \leq \sqrt{C} \sum_{m=d}^{\infty}(\alpha(p-1))^{\frac{m}{2}}<\infty .
\end{aligned}
$$

The converse is not true. For instance, the function $g(x)=|x|-\sqrt{\frac{2}{\pi}}$ belongs to $L^{p}(\mathbb{R}, \gamma)$ for all $p \geq 2$ and its Hermite coefficients satisfy

$$
(2 m) ! c_{2 m}^{2}=\frac{2(2 m) !}{\pi 2^{2 m}(m !)^{2}(2 m-1)^{2}} \sim \frac{2}{(2 m-1)^{2} \pi \sqrt{\pi m}} .
$$

Proof Theorem 3.1.5. For the convergence of the law of $Y_{n}$ to the law of $\sigma B$ in $C([0, T])$, we need:

(A) Convergence in law of the finite-dimensional distributions: this follows again from the Fourth Moment Theorem. Here we only need $g \in L^{2}(\mathbb{R}, \gamma)$ and condition (i).

(B) Tightness: This follows from the moment estimate:

$$
\left\|Y_{n}(t)-Y_{n}(s)\right\|_{L^{p}(\Omega)} \leq c|t-s|^{1 / 2},
$$

for some $p>2$, which is proved using techniques of Malliavin calculus.

Notice that when $g=\sum_{m=d}^{M} c_{m} H_{m}$, by hypercontractivity,

$$
\left\|Y_{n}(t)-Y_{n}(s)\right\|_{L^{p}(\Omega)} \leq c_{M, p}\left\|Y_{n}(t)-Y_{n}(s)\right\|_{L^{2}(\Omega)}
$$

and condition (ii) is not needed.

The proof of the moment estimates (3.2) will be done in several steps:

Step 1: Embedding the sequence $\left(\xi_{k}\right)_{k \geq 1}$ in the Wiener space.

Recall that $\xi=\left(\xi_{n}\right)_{n \in \mathbb{Z}}$ is a centered Gaussian stationary sequence with unit variance and covariance

$$
\rho(k)=\mathbb{E}\left[\xi_{n} \xi_{n+k}\right], \quad k \in \mathbb{Z} .
$$


Consider a sequence $e_{k} \in L^{2}([0, T])$ such that for each $j, k \in \mathbb{Z}$,

$$
\rho(j-k)=\left\langle e_{j}, e_{k}\right\rangle_{L^{2}([0, T])}=\int_{0}^{T} e_{j}(s) e_{k}(s) d s .
$$

Then,

$$
\left(\xi_{k}\right)_{k \in \mathbb{Z}} \stackrel{\text { law }}{=}\left(B\left(e_{k}\right)\right)_{k \in \mathbb{Z}}
$$

So, we can assume that $\xi_{k}=B\left(e_{k}\right)=\int_{0}^{T} e_{k}(s) d B_{s}$.

Step 2: Shift operator.

Let $g \in L^{2}(\mathbb{R}, \gamma)$ be a function of Hermite rank $d \geq 1$ and expansion

$$
g(x)=\sum_{m=d}^{\infty} c_{m} H_{m}(x) .
$$

For any $1 \leq k \leq d$, consider the function $g_{k}$ defined by

$$
g_{k}(x)=\sum_{m=d}^{\infty} c_{m} H_{m-k}(x)
$$

From $H_{m}=\delta^{k} H_{m-k}$, we deduce that

$$
\delta^{k} g_{k}(x)=g(x)
$$

Step 3: Representation of $g\left(\xi_{i}\right)$ as an iterated divergence.

We have

$$
g\left(\xi_{i}\right)=\delta^{d}\left(g_{d}\left(\xi_{i}\right) e_{i}^{\otimes d}\right) .
$$

Proof:

$$
g\left(\xi_{i}\right)=\sum_{m=d}^{\infty} c_{m} H_{m}\left(\xi_{i}\right)
$$

Then, for every $m \geq d$, using that multiple stochastic integrals are iterated divergences, we obtain

$$
\begin{aligned}
H_{m}\left(\xi_{i}\right) & =H_{m}\left(B\left(e_{i}\right)\right)=I_{m}\left(e_{i}^{\otimes m}\right)=\delta^{m}\left(e_{i}^{\otimes m}\right) \\
& =\delta^{d}\left(\delta^{m-d}\left(e_{i}^{\otimes(m-d)}\right) e_{i}^{\otimes d}\right)=\delta^{d}\left(I_{m-d}\left(e_{i}^{\otimes(m-d)}\right) e_{i}^{\otimes d}\right) \\
& =\delta^{d}\left(H_{m-d}\left(\xi_{i}\right) e_{i}^{\otimes d}\right) .
\end{aligned}
$$

Step 4: Regularization property of the shift.

Lemma 3.1.6. If $g \in L^{p}(\mathbb{R}, \gamma)$ for some $p \geq 2$ with Hermite rank $d$, then, for any $i \in \mathbb{Z}$ and $k=1, \ldots, d, g_{k}\left(\xi_{i}\right) \in \mathbb{D}^{k, p}$. 
Proof. Suppose $d=1$. We have

$$
D L^{-1} H_{m}\left(\xi_{i}\right)=-\frac{1}{m} H_{m}^{\prime}\left(\xi_{i}\right) e_{i}=-H_{m-1}\left(\xi_{i}\right) e_{i} .
$$

This implies

$$
g_{1}\left(\xi_{i}\right) e_{i}=-D L^{-1} g\left(\xi_{i}\right) .
$$

The result follows from the equivalence in $L^{p}$ of the operators $D$ and $(-L)^{1 / 2}$ (Meyer inequalities):

$$
\mathbb{E}\left(\left\|D^{2} L^{-1} g\left(\xi_{i}\right)\right\|_{L^{2}\left([0, T]^{2}\right)}^{p}\right) \leq c_{p} \mathbb{E}\left(\left|g\left(\xi_{i}\right)\right|^{p}\right) .
$$

Step 5: Proof of tightness using Malliavin calculus.

Assume $s=\frac{N_{1}}{n} \leq t=\frac{N_{2}}{n}$. We can write, using the continuity of the iterated divergence (see (1.11)),

$$
\begin{aligned}
& \left\|Y_{n}(t)-Y_{n}(s)\right\|_{L^{p}(\Omega)}=\frac{1}{\sqrt{n}}\left\|\sum_{i=N_{1}+1}^{N_{2}} g\left(\xi_{i}\right)\right\|_{L^{p}(\Omega)} \\
& =\frac{1}{\sqrt{n}}\left\|\sum_{i=N_{1}+1}^{N_{2}} \delta^{d}\left(g_{d}\left(\xi_{i}\right) e_{i}^{\otimes d}\right)\right\|_{L^{p}(\Omega)} \\
& \leq c_{p, d} \sum_{k=0}^{d} \frac{1}{\sqrt{n}}\left\|\sum_{i=N_{1}+1}^{N_{2}} D^{k}\left(g_{d}\left(\xi_{i}\right) e_{i}^{\otimes d}\right)\right\|_{L^{p}\left(\Omega ; L^{2}\left([0 . T]^{k+d}\right)\right.} \\
& \quad=c_{p, d} \sum_{k=0}^{d}\left\|\frac{1}{n} \sum_{i, j=N_{1}+1}^{N_{2}}\left\langle D^{k}\left(g_{d}\left(\xi_{i}\right)\right), D^{k}\left(g_{d}\left(\xi_{j}\right)\right)\right\rangle_{L^{2}\left([0, T]^{k}\right)} \rho(i-j)^{d}\right\|_{L^{\frac{p}{2}(\Omega)}}^{1 / 2} .
\end{aligned}
$$

Notice that, from Lemma 3.1.6,

$$
\sup _{j} \mathbb{E}\left[\left\|D^{k}\left(g_{d}\left(\xi_{i}\right)\right)\right\|_{L^{2}\left([0, T]^{k}\right)}^{p}\right]<\infty, \quad 0 \leq k \leq d
$$

because $\mathbb{E}\left[\left|g\left(\xi_{i}\right)\right|^{p}\right]<\infty$. As a consequence, deduce

$$
\left\|Y_{n}(t)-Y_{n}(s)\right\|_{L^{p}(\Omega)} \leq C\left(\frac{1}{n} \sum_{i, j=N_{1}+1}^{N_{2}}|\rho(i-j)|^{d}\right)^{1 / 2} .
$$

Finally, the change of indices $(i, j) \rightarrow(i, j+h)$ leads to

$$
\frac{1}{n} \sum_{i, j=N_{1}+1}^{N_{2}}|\rho(i-j)|^{d} \leq C \frac{N_{2}-N_{1}}{n} \sum_{h \in \mathbb{Z}}|\rho(h)|^{d}=C(t-s) .
$$

This completes the proof of the moment estimates. 


\subsubsection{Rate of convergence}

Recall that $\left(\xi_{k}\right)_{k \in \mathbb{Z}}$ is a centered Gaussian stationary sequence with unit variance and covariance $\rho(k)=\mathbb{E}\left[\xi_{n} \xi_{n+k}\right], k \in \mathbb{Z}$. Let

$$
F_{n}=\frac{1}{\sqrt{n}} \sum_{k=1}^{n} g\left(\xi_{k}\right)
$$

where $g$ has Hermite rank $d \geq 2$ and $\sum_{k \in \mathbb{Z}}|\rho(k)|^{d}<\infty$. Then, we know that

$$
F_{n}:=\frac{1}{\sqrt{n}} \sum_{k=1}^{n} g\left(\xi_{k}\right) \stackrel{\mathcal{L}}{\rightarrow} N\left(0, \sigma^{2}\right),
$$

where $\sigma^{2}=\sum_{m=d}^{\infty} c_{m}^{2} m ! \sum_{k \in \mathbb{Z}} \rho(k)^{m}$. Assume $\sigma^{2}>0$. Define $\sigma_{n}^{2}=$ $\operatorname{Var}\left(F_{n}\right)$ and consider the normalized sequence

$$
Z_{n}=\frac{F_{n}}{\sigma_{n}}
$$

We are interested in the rate of convergence to zero of $d_{T V}\left(Z_{n}, Z\right)$ where $Z \sim N(0,1)$. A basic estimated is given by the following proposition (see $(1.17))$.

Proposition 3.1.2. Suppose that $F \in \mathbb{D}^{1,2}$ satisfies $F=\delta(u)$, where $u$ belongs to $\operatorname{Dom} \delta$ and $\mathbb{E}\left[F^{2}\right]=1$. Then, if $Z \sim N(0,1)$,

$$
d_{T V}(F, Z) \leq 2 \sqrt{\operatorname{Var}\langle D F, u\rangle_{\mathfrak{H}}}
$$

We will discuss here only the case of Hermite rank $d=2$. Consider the particular function $g(x)=H_{2}(x)=x^{2}-1$. With the assumption $\xi_{k}=B\left(e_{k}\right)$, we can write

$$
Z_{n}=\frac{1}{\sigma_{n} \sqrt{n}} \sum_{j=1}^{n}\left[B^{2}\left(e_{j}\right)-1\right]=\frac{1}{\sigma_{n} \sqrt{n}} \sum_{j=1}^{n} \delta\left(B\left(e_{j}\right) e_{j}\right) .
$$

So, $Z_{n}=\delta\left(u_{n}\right)$, where

$$
u_{n}=\frac{1}{\sigma_{n} \sqrt{n}} \sum_{j=1}^{n} B\left(e_{j}\right) e_{j}
$$

Then,

$$
D Z_{n}=\frac{2}{\sigma_{n} \sqrt{n}} \sum_{j=1}^{n} B\left(e_{j}\right) e_{j}
$$

and

$$
\left\langle D Z_{n}, u_{n}\right\rangle_{\mathfrak{H}}=\frac{2}{n \sigma_{n}^{2}} \sum_{i, j=1}^{n} B\left(e_{i}\right) B\left(e_{j}\right) \rho(i-j) .
$$


As a consequence

$$
\begin{aligned}
& \operatorname{Var}\left(\left\langle D Z_{n}, u_{n}\right\rangle_{\mathfrak{H}}\right) \\
& =\frac{4}{n^{2} \sigma_{n}^{4}} \sum_{i_{1}, i_{2}, i_{3}, i_{4}=1}^{n} \operatorname{Cov}\left(B\left(e_{i_{1}}\right) B\left(e_{i_{2}}\right), B\left(e_{i_{3}}\right) B\left(e_{i_{4}}\right)\right) \rho\left(i_{1}-i_{2}\right) \rho\left(i_{3}-i_{4}\right) \\
& =\frac{4}{n^{2} \sigma_{n}^{4}} \sum_{i_{1}, i_{2}, i_{3}, i_{4}=1}^{n}\left[\rho\left(i_{1}-i_{3}\right) \rho\left(i_{2}-i_{4}\right) \rho\left(i_{1}-i_{2}\right) \rho\left(i_{3}-i_{4}\right)\right. \\
& \left.\quad+\rho\left(i_{1}-i_{4}\right) \rho\left(i_{2}-i_{3}\right) \rho\left(i_{1}-i_{2}\right) \rho\left(i_{3}-i_{4}\right)\right] \\
& =V_{n}^{(1)}+V_{n}^{(2)} .
\end{aligned}
$$

Both summands are similar and can be treated in the same way. Focusing only on the first summand, we make the change of variable $i_{1}-i_{2}=k_{1}$, $i_{3}-i_{4}=k_{2}$ and $i_{1}-i_{3}=k_{3}$ and we obtain

$$
V_{n}^{(1)} \leq \frac{C}{n} \sum_{\left|k_{i}\right| \leq n, i=1,2,3}\left|\rho\left(k_{1}\right) \rho\left(k_{2}\right) \rho\left(k_{3}\right) \rho\left(k_{2}+k_{3}-k_{1}\right)\right| .
$$

Set $\rho_{n}(k)=|\rho(k)| \mathbf{1}_{\{|k| \leq n\}}$. Then, by Hölder's inequality,

$$
\begin{aligned}
V_{n}^{(1)} & \leq \frac{C}{n}\left\langle\rho_{n},\left(\rho_{n} * \rho_{n}\right) * \rho_{n}\right\rangle_{\ell^{2}(\mathbb{Z})} \\
& \leq \frac{C}{n}\left\|\rho_{n}\right\|_{\ell^{4 / 3}(\mathbb{Z})}\left\|\left(\rho_{n} * \rho_{n}\right) * \rho_{n}\right\|_{\ell^{4}(\mathbb{Z})} .
\end{aligned}
$$

Applying Young's convolution inequality, yields

$$
\left.V_{n}^{(1)}\right) \leq \frac{C}{n}\left\|\rho_{n}\right\|_{\ell^{4 / 3}(\mathbb{Z})}^{4} \leq \frac{C}{n}\left(\sum_{|k| \leq n}|\rho(k)|^{\frac{4}{3}}\right)^{3} .
$$

Therefore in the case $g=H_{2}$ we have proved the following estimate

$$
d_{T V}\left(Z_{n}, Z\right) \leq \frac{C}{\sqrt{n}}\left(\sum_{|k| \leq n}|\rho(k)|^{\frac{4}{3}}\right)^{\frac{3}{2}}
$$

To handle the case of a general function $g$, let us recall the definition of the Sobolev spaces in the context of the one-dimensional Gaussian analysis. For any $m \geq 1$ and $p \geq 2, \mathbb{D}^{m, p}(\mathbb{R}, \gamma)$ is the class of functions $g \in L^{p}(\mathbb{R}, \gamma)$ which are $m$ times weakly differentiable and $g^{(k)} \in L^{p}(\mathbb{R}, \gamma)$ for all $1 \leq k \leq m$. 
Theorem 3.1.7 (Nourdin, Nualart and Peccati [15]). If $g \in \mathbb{D}^{1,4}$ has Hermite rank 2, then

$$
d_{T V}\left(Z_{n}, Z\right) \leq \frac{C}{\sqrt{n}}\left(\sum_{|k| \leq n}|\rho(k)|\right)^{\frac{1}{2}}+\frac{C}{\sqrt{n}}\left(\sum_{|k| \leq n}|\rho(k)|^{\frac{4}{3}}\right)^{\frac{3}{2}} .
$$

Nualart and Zhou [24] proved this result assuming $g \in \mathbb{D}^{4,4}$ and using Poincaré inequality and integration by parts. The proof for $g \in \mathbb{D}^{1,4}$ is based on Gebelein inequality.

Some ingredients of the proof of Theorem 3.1.7: We can represent $g\left(B\left(e_{i}\right)\right)$ as

$$
g\left(B\left(e_{i}\right)\right)=\delta\left(g_{1}\left(B\left(e_{i}\right)\right) e_{i}\right),
$$

where $g_{1}(x)=\sum_{m=2}^{\infty} c_{m} H_{m-1}(x)$. With this representation, we can write $Z_{n}=\delta\left(u_{n}\right)$, where

$$
u_{n}=\frac{1}{\sigma_{n} \sqrt{n}} \sum_{i=1}^{n} g_{1}\left(B\left(e_{i}\right)\right) e_{i}
$$

and

$$
\left\langle D Z_{n}, u_{n}\right\rangle_{\mathfrak{H}}=\frac{1}{n \sigma_{n}^{2}} \sum_{i, j=1}^{n} g^{\prime}\left(B\left(e_{i}\right)\right) g_{1}\left(B\left(e_{j}\right)\right) \rho(i-j) .
$$

Then, we can use two different approaches:

(i) If $g \in \mathbb{D}^{4,4}$, we can use Poincaré inequality:

$$
\operatorname{Var}\left(\left\langle D Z_{n}, u\right\rangle_{\mathfrak{H}}\right) \leq \mathbb{E}\left[\left\|D\left(\left\langle D Z_{n}, u\right\rangle_{\mathfrak{H}}\right)\right\|_{\mathfrak{H}}^{2}\right]
$$

and apply twice the duality between $D$ and $\delta$ (see Nualart and Zhou $[24])$.

(ii) Another option is to write

$$
\begin{aligned}
& \operatorname{Var}\left(\left\langle D Z_{n}, u_{n}\right\rangle_{\mathfrak{H}}\right)=\frac{1}{n^{2} \sigma_{n}^{4}} \\
& \times \sum_{\substack{i_{j}=1 \\
1 \leq j \leq 4}}^{n} \operatorname{Cov}\left(g^{\prime}\left(B\left(e_{i_{1}}\right)\right) g_{1}\left(B\left(e_{i_{2}}\right)\right), g^{\prime}\left(B\left(e_{i_{3}}\right)\right) g_{1}\left(B\left(e_{i_{4}}\right)\right)\right) \rho\left(i_{1}-i_{2}\right) \rho\left(i_{3}-i_{4}\right),
\end{aligned}
$$

and use Gebelein's inequality (Theorem 3.1.8 below) to estimate the covariances. 
Theorem 3.1.8 (Gebelein inequality). Let $\mathfrak{H}_{1}$ and $\mathfrak{H}_{2}$ be subspaces of $\mathfrak{H}$ and denote by $B_{1}$ and $B_{2}$ the restrictions of $B$ to $\mathfrak{H}_{1}$ and $\mathfrak{H}_{2}$, respectively. Consider centered and square integrable functionals $F_{i}\left(B_{i}\right), i=1,2$. Then,

$$
\begin{aligned}
\left|\mathbb{E}\left[F_{1}\left(B_{1}\right) F_{2}\left(B_{2}\right)\right]\right| & \leq \sup _{\varphi_{1} \in \mathfrak{H}_{1}, \varphi_{2} \in \mathfrak{H}_{2},\left\|\varphi_{1}\right\|_{\mathfrak{H}}=\left\|\varphi_{2}\right\|_{\mathfrak{H}}=1}\left|\left\langle\varphi_{1}, \varphi_{2}\right\rangle_{\mathfrak{H}}\right| \\
& \times \sqrt{\operatorname{Var}\left(F_{1}\left(B_{1}\right)\right) \operatorname{Var}\left(F_{2}\left(B_{2}\right)\right)} .
\end{aligned}
$$

Applying Gebelein's inequality in (3.5), we have

$$
\begin{aligned}
\operatorname{Var}\left(\left\langle D Z_{n}, u_{n}\right\rangle_{\mathfrak{H}}\right) & =\frac{C}{n^{2}} \sum_{i_{1}, i_{2}, i_{3}, i_{4}=1}^{n}\left|\rho\left(i_{1}-i_{2}\right) \rho\left(i_{3}-i_{4}\right)\right| \\
& \times \max \left\{\left|\rho\left(i_{1}-i_{3}\right)\right|,\left|\rho\left(i_{1}-i_{4}\right)\right|,\left|\rho\left(i_{2}-i_{3}\right)\right|,\left|\rho\left(i_{2}-i_{4}\right)\right|\right\} \\
& \leq \frac{C}{n} \sum_{\left|k_{i}\right| \leq n, i=1,2,3}\left|\rho\left(k_{1}\right) \rho\left(k_{2}\right) \rho\left(k_{3}\right)\right|=\frac{C}{n}\left(\sum_{\mid k_{\mid} \leq n}|\rho(k)|\right)^{3} .
\end{aligned}
$$

This gives the rate

$$
d_{T V}\left(Z_{n}, Z\right) \leq \frac{C}{\sqrt{n}}\left(\sum_{|k| \leq n}|\rho(k)|\right)^{\frac{3}{2}},
$$

which was proved by Nourdin, Peccati and Yang in [18]. The proof of (3.4) is more involved (see [15]]).

Example: Suppose that $g(x)=|x|^{p}-\mathbb{E}\left[|Z|^{p}\right]$, for any $p \geq 1$. Consider the particular case where $\rho(k) \sim k^{-\alpha}$ for some $\alpha>0$. Then, condition $\sum_{k \in \mathbb{Z}} \rho(k)^{2}<\infty$ means $\alpha>\frac{1}{2}$, and the rate is

$$
d_{T V}\left(Z_{n}, Z\right) \leq C \times \begin{cases}n^{-\frac{1}{2}} & \text { if } \alpha>1 \\ n^{-\frac{1}{2}} \sqrt{\log n} & \text { if } \alpha=1 \\ n^{-\frac{\alpha}{2}} & \text { if } \alpha \in\left[\frac{2}{3}, 1\right) \\ n^{1-2 \alpha} & \text { if } \alpha \in\left(\frac{1}{2}, \frac{2}{3}\right) .\end{cases}
$$

For functions with Hermite rank $d \geq 3$, Nualart-Zhou [24] proved that for $g \in \mathbb{D}^{3 d-2,4}(\mathbb{R}, \gamma)$, we have the estimate

$$
\begin{aligned}
d_{\mathrm{TV}}\left(Z_{n}, Z\right) \leq & C_{1} n^{-\frac{1}{2}} \sum_{|k| \leq n}|\rho(k)|^{d-1}\left(\sum_{|k| \leq n}|\rho(k)|^{2}\right)^{\frac{1}{2}} \\
& +C_{2} n^{-\frac{1}{2}}\left(\sum_{|k| \leq n}|\rho(k)|^{2}\right)^{\frac{1}{2}}\left(\sum_{|k| \leq n}|\rho(k)|\right)^{\frac{1}{2}},
\end{aligned}
$$


where $C_{2}=0$ if $g=H_{d}$. The proof is based on Poincaré inequality, integration by parts and the Brascamp-Lieb inequaity, stated in the next theorem, which is a generalization of Hölder and Young's convolution inequalities.

Theorem 3.1.9 (Brascamp-Lieb inequality). The following inequality holds true

$$
\sum_{\mathbf{k} \in \mathbb{Z}^{M}} \prod_{j=1}^{N} \rho_{j}\left(\mathbf{k} \cdot v_{j}\right) d \mathbf{k} \leq C \prod_{j=1}^{N}\left(\sum_{k \in \mathbb{Z}} \rho_{j}(k)^{1 / p_{j}}\right)^{p_{j}},
$$

where $\rho_{j}(x) \geq 0, v_{j}$ are nonzero vectors in $\mathbb{R}^{M}$, and the $p_{j}$ satisfy:

(i) $\sum_{j=1}^{N} p_{j}=M$,

(ii) For any $I \subset\{1, \ldots, M\}, \sum_{j \in I} p_{j} \leq \operatorname{dim}\left(\operatorname{Span}\left\{v_{j}, j \in I\right\}\right)$.

As an application we can easily derive the estimate (3.3):

$$
\sum_{\left|k_{1}\right|,\left|k_{2}\right|,\left|k_{3}\right| \leq n}\left|\rho\left(k_{1}\right) \rho\left(k_{2}\right) \rho\left(k_{3}\right) \rho\left(k_{1}-k_{2}+k_{3}\right)\right| \leq C\left(\sum_{|k| \leq n}|\rho(k)|^{\frac{4}{3}}\right)^{3} .
$$

Indeed, in this case $M=3, N=4, v_{1}=(1,0,0), v_{2}=(0,1,0)$, $v_{3}=(0,0,1)$ and $v_{4}=(1,-1,1)$. The $p_{j}$ must satisfy:

$$
p_{1}+p_{2}+p_{3}+p_{4}=3,
$$

and for all $j \neq k, p_{j} \leq 1$ and $p_{j}+p_{k} \leq 2$. Choosing $p_{j}=\frac{3}{4}$, we obtain the bound (3.3).

\subsubsection{Optimal rate of convergence}

By means of an intensive application of the Malliavin-Stein's method, Nourdin and Peccati obtained the following optimal rate of convergence in total variation for random variables in a fixed chaos.

Theorem 3.1.10 (Nourdin and Peccati $[\mathbf{1 7}]$ ). Assume $\mathbb{E}\left[F^{2}\right]=1$ and $F$ belongs a fixed Wiener chaos of order $d \geq 1$. Then,

$$
c \Phi(F) \leq d_{T V}(F, Z) \leq C \Phi(F),
$$

where $\Phi(F)=\max \left(\left|\mathbb{E}\left[F^{3}\right]\right|, \mathbb{E}\left[F^{4}\right]-3\right)$.

In the framework of the Breuer-Major theorem, this yields (see Biermé, Bonami, Nourdin and Peccati [2]):

$$
d_{T V}\left(Z_{n}, Z\right) \sim \frac{C}{\sqrt{n}}\left(\sum_{|k| \leq n}|\rho(k)|^{\frac{3}{2}}\right)^{2} .
$$


if $g=H_{2}$ and

$$
\begin{aligned}
d_{T V}\left(F_{n}, Z\right) \sim & \frac{c_{1}}{n}\left(\sum_{|k| \leq n}|\rho(k)|^{d-1}\right)^{2} \sum_{|k| \leq n}|\rho(k)|^{2} \\
& +\frac{c_{2}}{n}\left(\sum_{|k| \leq n}|\rho(k)|^{\frac{2 d}{3}}\right)^{3} \mathbf{1}_{\{d \text { even }\}}
\end{aligned}
$$

for $g=H_{d}, d \geq 3$.

Nualart and Zhou [24] proved (3.6) for $g$ of rank $d=2$ in $\mathbb{D}^{6,8}(\mathbb{R}, \gamma)$. We conjecture that the estimate holds when $g \in \mathbb{D}^{3,8}(\mathbb{R}, \gamma)$. The main ingredient in the proof of this result is the following theorem that generalizes Theorem 3.1.10 to the case of random variables that do not belong to a fixed chaos.

We will make use of the notation $D_{u} F=\langle D F, u\rangle_{\mathfrak{H}}$.

Theorem 3.1.11. Assume $F=\delta(u) \in \mathbb{D}^{3,2}$ with $u \in \operatorname{Dom} \delta$ and $\mathbb{E}\left(F^{2}\right)=1$. Then if $Z \sim N(0,1)$,

$$
\begin{aligned}
d_{T V}(F, Z) \leq & (8+\sqrt{32 \pi}) \operatorname{Var}\left(\langle D F, u\rangle_{\mathfrak{H}}\right)+\sqrt{2 \pi}\left|\mathbb{E}\left(F^{3}\right)\right| \\
& +\sqrt{32 \pi} \mathbb{E}\left(\left|D_{u}^{2} F\right|^{2}\right)+4 \pi \mathbb{E}\left(\left|D_{u}^{3} F\right|\right) .
\end{aligned}
$$

Proof. Let $h: \mathbb{R} \rightarrow[0,1]$ be a Borel function. Using Stein's equation, there exists an absolutely continuous function $f_{h}$ such that $\left\|f_{h}\right\|_{\infty} \leq \sqrt{\frac{\pi}{2}}$ and $\left\|f_{h}^{\prime}\right\|_{\infty} \leq 2$, satisfying

$$
I:=|\mathbb{E}(h(F))-\mathbb{E}(h(Z))|=\left|\mathbb{E}\left(f_{h}^{\prime}(F)-F f_{h}(F)\right)\right| .
$$

Applying duality yields

$$
I=\left|\mathbb{E}\left(f_{h}^{\prime}(F)\left(1-\langle D F, u\rangle_{\mathfrak{H}}\right)\right)\right| .
$$

Taking into account that $\mathbb{E}\left(\langle D F, u\rangle_{\mathfrak{H}}\right)=\mathbb{E}\left(F^{2}\right)=1$, we have

$$
I=\left|\mathbb{E}\left(\left(f_{h}^{\prime}(F)-\mathbb{E}\left(f_{h}^{\prime}(Z)\right)\right)\left(1-\langle D F, u\rangle_{\mathfrak{H}}\right)\right)\right| .
$$

We apply Stein's equation associated with $\varphi=f_{h}^{\prime}$ to obtain

$$
I=\left|\mathbb{E}\left(\left(f_{\varphi}^{\prime}(F)-F f_{\varphi}(F)\right)\left(1-\langle D F, u\rangle_{\mathfrak{H}}\right)\right)\right|
$$

where $\left\|f_{\varphi}\right\|_{\infty} \leq 4 \sqrt{\pi / 2}$ and $\left\|f_{\varphi}^{\prime}\right\|_{\infty} \leq 8$. We continue to apply duality in order to get

$$
\begin{aligned}
I & =\left|\mathbb{E}\left(f_{\varphi}^{\prime}(F)\left(1-D_{u} F\right)-\left\langle u, D\left(f_{\varphi}(F)\left(1-D_{u} F\right)\right)\right\rangle_{\mathfrak{H}}\right)\right| \\
& =\left|\mathbb{E}\left(f_{\varphi}^{\prime}(F)\left(1-D_{u} F\right)^{2}\right)+\mathbb{E}\left(f_{\varphi}(F) D_{u}^{2} F\right)\right| \\
& \leq 8 \mathbb{E}\left[\left(1-D_{u} F\right)^{2}\right]+\left|\mathbb{E}\left(\left(f_{\varphi}(F)-\mathbb{E}\left(f_{\varphi}(Z)\right)\right) D_{u}^{2} F\right)\right|+\left|\mathbb{E}\left(f_{\varphi}(Z)\right) \mathbb{E}\left(D_{u}^{2} F\right)\right| \\
& =: I_{1}+I_{2}+I_{3} .
\end{aligned}
$$


For the term $I_{1}$, we have

$$
I_{1}=8 \operatorname{Var}\left(\langle D F, u\rangle_{\mathfrak{H}}\right) .
$$

For the term $I_{3}$, taking into account that

$$
\mathbb{E}\left(D_{u}^{2} F\right)=\mathbb{E}\left(\langle u, D F\rangle_{\mathfrak{H}} \delta(u)\right)=\frac{1}{2} \mathbb{E}\left(\left\langle u, D F^{2}\right\rangle_{\mathfrak{H}}\right)=\frac{1}{2} \mathbb{E}\left(F^{3}\right),
$$

we obtain

$$
I_{3} \leq 2 \sqrt{\pi / 2}\left|\mathbb{E}\left(F^{3}\right)\right|
$$

Finally, for the term $I_{2}$, applying Stein's equation associated with $\psi=f_{\varphi}$ yields

$$
\begin{aligned}
I_{2} & =\left|\mathbb{E}\left(\left(f_{\psi}^{\prime}(F)-F f_{\psi}(F)\right) D_{u}^{2} F\right)\right| \\
& \leq\left|\mathbb{E}\left(f_{\psi}^{\prime}(F)\left(D_{u}^{2} F-D_{u} F D_{u}^{2} F\right)\right)\right|+\left|\mathbb{E}\left(f_{\psi}(F) D_{u}^{3} F\right)\right|,
\end{aligned}
$$

where $f_{\psi}$ satisfies $\left\|f_{\psi}\right\|_{\infty} \leq 16 \sqrt{\pi / 2}$ and $\left\|f_{\psi}^{\prime}\right\|_{\infty} \leq 4 \pi$. Finally,

$$
\mathbb{E}\left(\left|D_{u}^{2} F-D_{u} F D_{u}^{2} F\right|\right) \leq \frac{1}{2}\left(\mathbb{E}\left(\left|D_{u}^{2} F\right|^{2}\right)+\mathbb{E}\left(\left|1-D_{u} F\right|^{2}\right)\right) .
$$

The proof is now complete.

\subsection{Fractional Brownian motion}

The fractional Brownian motion $(\mathrm{fBm}) B^{H}=\left(B_{t}^{H}\right)_{t \geq 0}$ is a zero mean Gaussian process with covariance

$$
\mathbb{E}\left(B_{s}^{H} B_{t}^{H}\right)=R_{H}(s, t)=\frac{1}{2}\left(s^{2 H}+t^{2 H}-|t-s|^{2 H}\right) .
$$

$H \in(0,1)$ is called the Hurst parameter.

The covariance formula implies $\mathbb{E}\left(B_{t}^{H}-B_{s}^{H}\right)^{2}=|t-s|^{2 H}$. As a consequence, for any $\gamma<H$, with probability one, the trajectories $t \rightarrow B_{t}^{H}(\omega)$ are Hölder continuous of order $\gamma$ :

$$
\left|B_{t}^{H}(\omega)-B_{s}^{H}(\omega)\right| \leq G_{\gamma, T}(\omega)|t-s|^{\gamma}, \quad s, t \in[0, T] .
$$

For $H=\frac{1}{2}, B^{\frac{1}{2}}$ is a Brownian motion.

Properties of the fractional Brownian motion:

1) The fractional Brownian motion has the following self-similarity property. For all $a>0$, the processes $\left(a^{-H} B_{a t}^{H}\right)_{t \geq 0}$ and $\left(B_{t}^{H}\right)_{t \geq 0}$ have the same probability distribution (they are fractional Brownian motions with Hurst parameter $H$ ). 
2) Unlike Brownian motion, the fractional Brownian motion has correlated increments. More precisely, for $H \neq \frac{1}{2}$, we can write

$$
\begin{aligned}
\rho(n)= & \mathbb{E}\left(B_{1}^{H}\left(B_{n+1}^{H}-B_{n}^{H}\right)\right)=\frac{1}{2}\left((n+1)^{2 H}+(n-1)^{2 H}-2 n^{2 H}\right) \\
& \sim H(2 H-1) n^{2 H-2},
\end{aligned}
$$

as $n \rightarrow \infty$.

(ii) If $H>\frac{1}{2}$, then $\rho(n)>0$ and $\sum_{n} \rho(n)=\infty$ (long memory).

(iii) If $H<\frac{1}{2}$, then $\rho(n)<0$ (intermittency) and $\sum_{n}|\rho(n)|<\infty$.

3) The fractional Brownian motion has finite $\frac{1}{H}$-variation: Fix $T>0$. Set $t_{i}=\frac{i T}{n}$ for $1 \leq i \leq n$ and define $\Delta B_{t_{i}}^{H}=B_{t_{i}}^{H}-B_{t_{i-1}}^{H}$. Then, as $n \rightarrow \infty$,

$$
\sum_{i=1}^{n}\left|\Delta B_{t_{i}}^{H}\right|^{\frac{1}{H}} \stackrel{L^{2}}{\stackrel{(\Omega), a . s .}{\longrightarrow}} c_{H} T,
$$

where $c_{H}=\mathbb{E}\left[\left|B_{1}^{H}\right|^{\frac{1}{H}}\right]$.

Proof. By the self-similarity, $\sum_{i=1}^{n}\left|\Delta B_{t_{i}}^{H}\right|^{\frac{1}{H}}$ has the same law as

$$
\frac{T}{n} \sum_{i=1}^{n}\left|B_{i}^{H}-B_{i-1}^{H}\right|^{\frac{1}{H}} .
$$

The sequence $\left(B_{i}^{H}-B_{i-1}^{H}\right)_{i \geq 1}$ is stationary and ergodic. Therefore, the Ergodic Theorem implies the desired convergence.

\subsubsection{Fractional noise}

Let $\xi_{k}=B_{k}^{H}-B_{k-1}^{H}$. The sequence $\left(\xi_{k}\right)_{k \geq 1}$ is Gaussian, stationary and centered with covariance

$$
\rho_{h}(k)=\frac{1}{2}\left(|k+1|^{2 H}+|k-1|^{2 H}-2|k|^{2 H}\right) .
$$

We have $\rho(k) \sim H(2 H-1) k^{2 H-2}$ as $k \rightarrow \infty$. Then, for any integer $d \geq 2$ such that $H<1-\frac{1}{2 d}$, we have

$$
\sum_{v \in \mathbb{Z}}|\rho(v)|^{d}<\infty .
$$

By the Breuer-Major theorem, that if $g \in L^{p}(\mathbb{R}, \gamma), p>2$, has Hermite rank $d$ and $H<1-\frac{1}{2 d}$

$$
\frac{1}{\sqrt{n}} \sum_{k=1}^{\lfloor n t\rfloor} g\left(\Delta_{k} B^{H}\right) \stackrel{\mathcal{L}}{\rightarrow} \sigma_{H} B,
$$

where $\sigma_{H}^{2}=\sum_{m=d}^{\infty} c_{m}^{2} m ! \sum_{k \in \mathbb{Z}} \rho_{H}(k)^{m}$ and $B$ is a Brownian motion. 


\subsubsection{Asymptotic behavior of the the $q$-variation of the $\mathrm{fBm}$}

For a real $q \geq 1$, set $c_{q}=\mathbb{E}\left[|Z|^{q}\right]$, where $Z \sim N(0,1)$. By self-similarity and the Ergodic Theorem, the normalized $p$-variaton satisfies

$$
n^{p H-1} \sum_{k=1}^{n}\left|B_{\frac{k}{n}}^{H}-B_{\frac{k-1}{n}}^{H}\right|^{p} \stackrel{\mathcal{L}}{=} \frac{1}{n} \sum_{k=1}^{n}\left|B_{k}^{H}-B_{k-1}^{H}\right|^{p \stackrel{a . s .}{\rightarrow}} c_{p} .
$$

Then, if $H<\frac{3}{4}$, using that $|x|^{p}-c_{p}$ has Hermite rank 2, the Breuer-Major theorem leads to the following Central Limit Theorem:

$$
\frac{1}{\sqrt{n}} \sum_{k=1}^{\lfloor n t\rfloor}\left[n^{p H}\left|B_{\frac{k}{n}}^{H}-B_{\frac{k-1}{n}}^{H}\right|^{p}-c_{p}\right] \stackrel{\mathcal{L}}{\rightarrow} \sigma_{H, p} B .
$$

We can also deduce from the previous results, the following rate of convergence of the quadratic variation. The sequence $Z_{n}=$ $\frac{1}{\sigma_{n}} \sum_{k=1}^{n}\left[\left(B_{k}^{H}-B_{k-1}^{H}\right)^{2}-1\right]$, with $\operatorname{Var}\left(Z_{n}\right)=1$, satisfies:

$$
d_{T V}\left(Z_{n}, Z\right) \sim c_{H} \times \begin{cases}n^{-\frac{1}{2}} & \text { if } H \in\left(0, \frac{2}{3}\right) \\ n^{-\frac{1}{2}}(\log n)^{2} & \text { if } H=\frac{2}{3} \\ n^{6 H-\frac{9}{2}} & \text { if } H \in\left(\frac{2}{3}, \frac{3}{4}\right) .\end{cases}
$$




\section{Chapter 4}

\section{Spatial averaging of SPDEs}

\subsection{Stochastic heat equation}

Consider the one-dimensional stochastic heat equation

$$
\frac{\partial u}{\partial t}=\frac{1}{2} \frac{\partial^{2} u}{\partial x^{2}}+\sigma(u) \frac{\partial^{2} W}{\partial t \partial x}, \quad x \in \mathbb{R}, \quad t \geq 0,
$$

with initial condition $u_{0}(x)=1$, driven by a space-time white noise $\frac{\partial^{2} W}{\partial t \partial x}$. We assume that $\sigma$ is a Lipschitz function such that $\sigma(1) \neq 0$.

The space-time white noise is formally defined as a Gaussian centered family of random variables $\left\{W(A), A \in \mathcal{B}\left(\mathbb{R}_{+} \times \mathbb{R}\right),|A|<\infty\right\}$ with covariance given by

$$
\mathbb{E}[W(A) W(B)]=|A \cap B| .
$$

Writing $W(A)=W\left(\mathbf{1}_{A}\right)$, we can extend $W$ to $\mathfrak{H}=L^{2}\left(\mathbb{R}_{+} \times \mathbb{R}\right)$, in such a way that

$$
W(h)=\int_{\mathbb{R}_{+} \times \mathbb{R}} h(t, x) W(d t, d x)
$$

is an isonormal Gaussian process on $\mathfrak{H}$. Moreover, $W(s, t)=W([0, s] \times$ $[0, t]), s, t \geq 0$ is a two-parameter Brownian motion.

\subsubsection{Stochastic integration}

For any $t \geq 0$, let $\mathcal{F}_{t}$ be the $\sigma$-algebra generated by $\{W(A), A \in$ $\mathcal{B}([0, t] \times \mathbb{R})\}$. A random field $u=\{u(t, x), t \geq 0, x \in \mathbb{R}\}$ is adapted if $u(t, x)$ is $\mathcal{F}_{t}$-measurable for each $t \geq 0$ and $x \in \mathbb{R}$. We can define the 
Itô-Walsh stochastic integral of adapted and measurable processes $u$ such that

$$
\int_{\mathbb{R}_{+} \times \mathbb{R}} \mathbb{E}\left(u^{2}(t, x)\right) d t d x<\infty
$$

in such a way that the following isometry property holds:

$$
\mathbb{E}\left(\left|\int_{\mathbb{R}_{+} \times \mathbb{R}} u(t, x) W(d t, d x)\right|^{2}\right)=\int_{\mathbb{R}_{+} \times \mathbb{R}} \mathbb{E}\left(u^{2}(t, x)\right) d t d x .
$$

Suppose that $\delta$ is the divergence operator in the framework of the Malliavin calculus for the isonormal Gaussian process $\{W(h), h \in \mathfrak{H}\}$. Then, If $v \in L^{2}\left(\Omega \times \mathbb{R}_{+} \times \mathbb{R}\right)$ is a square integrable adapted random field, $v$ belongs to the domain of $\delta$ and $\delta(v)$ coincides with the Itô-Walsh integral of $v$ :

$$
\delta(v)=\int_{\mathbb{R}_{+} \times \mathbb{R}} v(s, y) W(d s, d y) .
$$

\subsubsection{Mild solution}

The following is the fundamental result on the existence and uniqueness of a mild solution to the stochastic heat equation.

Theorem 4.1.1 (Walsh [29]). There is a unique mild solution, which is an adapted random field $u$ such that for all $p \geq 2$,

$$
\sup _{x \in \mathbb{R}} \sup _{0 \leq t \leq T} \mathbb{E}\left[|u(t, x)|^{p}\right]<\infty,
$$

and $u$ satisfies the integral equation:

$$
u(t, x)=1+\int_{0}^{t} \int_{\mathbb{R}} p_{t-s}(x-y) \sigma(u(s, y)) W(d s, d y),
$$

where $p_{t}(x)=\frac{1}{\sqrt{2 \pi}} \exp \left(-x^{2} / 2\right)$.

Moreover, we have

$$
\sup _{t \in[0, T]} \mathbb{E}\left(|u(t, x)-u(s, y)|^{p}\right) \leq C_{T, p}\left(|s-t|^{p / 4}+|x-y|^{p / 2}\right) .
$$

\subsubsection{Malliavin differentiability of the solution}

We will first show that the random variable $u(t, x)$ belongs to the space $\mathbb{D}^{1, p}$ and obtain bounds for the $p$-moments of the derivative.

Proposition 4.1.1. For each $(t, x) \in \mathbb{R}_{+} \times \mathbb{R}, u(t, x) \in \mathbb{D}^{1, p}$ for all $p \geq 2$ and the derivative $D_{s, y} u(t, x)$ satisfies 
(i) $D_{s, y} u(t, x)=0$ if $s>t$ and for $s \leq t$ :

$$
\begin{aligned}
D_{s, y} u(t, x)= & p_{t-s}(x, y) \sigma(u(s, y)) \\
& +\int_{s}^{t} \int_{\mathbb{R}} p_{t-r}(x, z) \Sigma(r, z) D_{s, y} u(r, z) W(d r, d z)
\end{aligned}
$$

where $\Sigma(r, z),(r, z) \in \mathbb{R}_{+} \times \mathbb{R}$, is an adapted and bounded process.

(ii) For all $t \in[0, T]$, and $p \geq 2$,

$$
\left\|D_{s, y} u(t, x)\right\|_{p} \leq C_{T, p} p_{t-s}(x-y)
$$

for almost all $(s, y) \in[0, t] \times \mathbb{R}$.

Remark: If the coefficient $\sigma$ is of class $C^{1}$ with bounded derivative, then $\Sigma(r, z)=\sigma^{\prime}(u(r, z))$.

Proof. The proof will be done in several steps:

Step 1: Consider the Picard approximations defined by $u_{0}(t, x)=1$, and for each integer $n \geq 0$ set

$$
u_{n+1}(t, x):=1+\int_{0}^{t} \int_{\mathbb{R}} p_{t-s}(x-y) \sigma\left(u_{n}(s, y)\right) W(d s, d y) .
$$

We know that, for each $p \geq 2$,

$$
\lim _{n \rightarrow \infty} \mathbb{E}\left(\left|u_{n}(t, x)-u(t, x)\right|^{p}\right)=0
$$

and also $\sup _{n} \sup _{t \in[0, T]} \sup _{x \in \mathbb{R}} \mathbb{E}\left(\left|u_{n}(t, x)\right|^{p}\right)<\infty$.

By induction we will show that for each $(t, x) \in[0, T] \times \mathbb{R}$ and for each $p \geq 2, u_{n}(t, x) \in \mathbb{D}^{1, p}$ and

$$
\left\|D_{s, y} u_{n}(t, x)\right\|_{p} \leq C_{T, p} p_{t-s}(x-y)
$$

for almost all $(s, y) \in[0, t] \times \mathbb{R}$.

Suppose that the induction hypothesis holds for all integers less than or equal to $n$. By the chain rule for Lipschitz functions (see Proposition 1.4.2), for each $(t, x) \in[0, T] \times \mathbb{R}, \sigma\left(u_{n}(t, x)\right)$ belongs to $\mathbb{D}^{1, p}$ and there exists a random variable $\Sigma_{n}$ bounded by the Lipschitz constant of $\sigma$, such that

$$
D\left(\sigma\left(u_{n}(t, x)\right)\right)=\Sigma_{n} D u_{n}(t, x) .
$$

If $\sigma$ is continuously differentiable, then $\Sigma_{n}=\sigma^{\prime}\left(u_{n}(t, x)\right)$. Then, applying the properties of the divergence operator (see (1.12), we deduce that 
$\int_{0}^{t} \int_{\mathbb{R}} p_{t-s}(x-y) \sigma\left(u_{n}(s, y)\right) W(d s, d y)$ is in $\mathbb{D}^{1, p}$ and

$$
\begin{aligned}
& D_{s, y} u_{n+1}(t, x) \\
= & D_{s, y}\left(\int_{0}^{t} \int_{\mathbb{R}} p_{t-r}(x-z) \sigma\left(u_{n}(r, z)\right) W(d r, d z)\right) \\
= & p_{t-s}(x-y) \sigma\left(u_{n}(s, y)\right)+\int_{r}^{t} \int_{\mathbb{R}} p_{t-r}(x-z) \Sigma_{n} D_{s, y} u_{n}(r, z) W(d r, d z)
\end{aligned}
$$

Now, let us estimate the $L^{p}$ norm of $D_{s, y} u_{n+1}(t, x)$.

$$
\begin{aligned}
& \mathbb{E}\left(\left|D_{s, y} u_{n+1}(t, x)\right|^{p}\right) \leq \sup _{r, z} \mathbb{E}\left(\left|\sigma\left(u_{n}(r, z)\right)\right|^{p}\right) p_{t-s}(x-y) \\
& \quad+\mathbb{E}\left(\left|\int_{s}^{t} \int_{\mathbb{R}} p_{t-r}(x-z) \Sigma_{n} D_{s, y} u_{n}(r, z) W(d r, d z)\right|^{p}\right) .
\end{aligned}
$$

The Burkholder-David-Gundy inequality yields, with a constant $c_{p}$,

$$
\begin{aligned}
& \mathbb{E}\left(\left|\int_{s}^{t} \int_{\mathbb{R}} p_{t-r}(x-z) \Sigma_{n} D_{s, y} u_{n}(r, z) W(d r, d z)\right|^{p}\right) \\
& \leq c_{p}^{p} \operatorname{Lip}(\sigma)^{p} \mathbb{E}\left(\left.\left.\left|\int_{s}^{t} \int_{\mathbb{R}} p_{t-r}^{2}(x-z)\right| D_{s, y} u_{n}(r, z)\right|^{2} d z d r\right|^{p / 2}\right) \\
& \leq c_{p}^{p} \operatorname{Lip}(\sigma)^{p}\left(\int_{s}^{t} \int_{\mathbb{R}} p_{t-r}^{2}(x-z)\left\|D_{s, y} u_{n}(r, z)\right\|_{p}^{2} d z d r\right)^{1 / 2},
\end{aligned}
$$

where $\operatorname{Lip}(\sigma)$ denotes the Lipschitz constant of $\sigma$. Therefore, the preceding displayed computation yields that

$$
\begin{aligned}
\left\|D_{s, y} u_{n+1}(t, x)\right\|_{p}^{2} \leq & K_{T, p}^{2} p_{t-s}^{2}(x-y) \\
& +c_{p}^{2} \operatorname{Lip}(\sigma)^{2} \int_{s}^{t} \int_{\mathbb{R}} p_{t-r}^{2}(x-z)\left\|D_{s, y} u_{n}(r, z)\right\|_{p}^{2} d z d r,
\end{aligned}
$$

where

$$
K_{T, p}:=\sup _{n} \sup _{(s, y) \in[0, T] \times \mathbb{R}^{d}}\left\|\sigma\left(u_{n}(s, y)\right)\right\|_{p}<\infty .
$$

By iterating this inequality, yields

$$
\begin{aligned}
& \left\|D_{s, y} u_{n+1}(r, z)\right\|_{p}^{2} \\
\leq & K_{T, p}^{2} p_{r-s}^{2}(z-y) \\
& +c_{p}^{2} \operatorname{Lip}(\sigma)^{2} \sum_{k=1}^{n-1} \int_{\Delta_{k-1}(s, t)} \int_{\mathbb{R}^{k-1}} \prod_{j=1}^{k-1} p_{r_{j-1}-r_{j}}^{2}\left(z_{j-1}-z_{j}\right) d \mathbf{z}_{k-1} d \mathbf{r}_{k-1}+R_{n},
\end{aligned}
$$


with the convention $r_{0}=t, z_{0}=x, r_{k-1}=s$ and $z_{k-1}=y$. Here $d \mathbf{z}_{k-1}$ means $d z_{1} \cdots d z_{k-1}$ and $\Delta_{k-1}(s, t)$ denotes the simplex $\left\{s \leq r_{k-1}<\cdots<\right.$ $\left.r_{1}<t\right\}$. The residual term $R_{n}$, taking into account that $u(0, x)=1$, can be expressed as

$$
R_{n}=c_{p}^{2} \operatorname{Lip}(\sigma)^{2} \sigma^{2}(1) \int_{\Delta_{n}(s, t)} \int_{\mathbb{R}^{n}} \prod_{j=1}^{n} p_{r_{j-1}-r_{j}}^{2}\left(z_{j-1}-z_{j}\right) d \mathbf{z}_{n} d \mathbf{r}_{n}
$$

with the same conventions as before. Then, using the fact that

$$
p_{r-s}^{2}(z)=\frac{C}{\sqrt{r-s}} p_{\frac{r-s}{2}}(z)
$$

and

$$
\int_{\mathbb{R}} p_{s}(x-y) p_{t}(y-z) d y=p_{s+t}(x-z),
$$

we have for the integrals of the product of heat kernels above,

$$
\begin{aligned}
& \int_{\Delta_{n}(s, t)} \int_{\mathbb{R}^{n}} \prod_{j=1}^{n} p_{r_{j-1}-r_{j}}^{2}\left(z_{j-1}-z_{j}\right) d \mathbf{z}_{n} d \mathbf{r}_{n} \\
\leq & C^{n} \int_{\Delta_{n}(s, t)} \int_{\mathbb{R}^{n}} \prod_{j=1}^{n}\left(r_{j-1}-r_{j}\right)^{-\frac{1}{2}} p_{\left(r_{j-1}-r_{j}\right) / 2}\left(z_{j-1}-z_{j}\right) d \mathbf{z}_{n} d \mathbf{r}_{n} \\
= & C^{n}(t-s)^{\frac{n-1}{2}} \frac{\Gamma\left(\frac{1}{2}\right)^{n+1}}{\Gamma\left(\frac{n+1}{2}\right)} p_{\frac{t-s}{2}}(x-y) \\
= & C^{n}(t-s)^{\frac{n}{2}} \frac{\Gamma\left(\frac{1}{2}\right)^{n+1}}{\Gamma\left(\frac{n+1}{2}\right)} p_{t-s}^{2}(x-y) .
\end{aligned}
$$

This allows us to complete the proof of (4.2).

Step 2: We know that $u_{n}(t, x)$ converges in $L^{p}(\Omega)$ to $u(t, x)$. The estimate (4.2) implies that

$$
\sup _{n} \mathbb{E}\left(\left\|D u_{n}(t, x)\right\|_{\mathfrak{H}}^{p}\right)<\infty
$$

By Proposition 1.4.1, this implies that $u(t, x)$ belongs to $\mathbb{D}^{1, p}$. Applying the operator $D$ to the equation satisfied by $u(t, x)$ we deduce point (i).

Step 3: Finally, it remains to show that the estimate (4.2) holds for $u(t, x)$. This follows from the fact that $D u_{n}(t, x)$ (by choosing a subsequence) converges in the weak topology of $L^{p}(\Omega ; \mathfrak{H})$ to $D u(t, x)$. 


\subsection{Space averages}

Fix $t \geq 0$. We are interested in the asymptotic behavior as $R \rightarrow \infty$ of the random variable

$$
\int_{-R}^{R} u(t, x) d x
$$

Because the process $x \rightarrow u(t, x)$ is stationary and the covariance decays fast, we expect a central limit theorem to hold for these space averages.

The mean of this variable is given by

$$
\mathbb{E}\left(\int_{-R}^{R} u(t, x) d x\right)=2 R
$$

Notice that

$$
\int_{-R}^{R} u(t, x) d-2 R=\int_{0}^{t} \int_{\mathbb{R}}\left(\int_{-R}^{R} p_{t-s}(x-y) d x\right) \sigma(u(s, y)) W(d s, d y) .
$$

Set

$$
f_{R}(s, y)=\int_{-R}^{R} p_{t-s}(x-y) d x .
$$

Notice that $f_{R}(s, y) \leq 1$ and

$$
\begin{aligned}
\int_{\mathbb{R}} f_{R}^{2}(s, y) d y & =\int_{\mathbb{R}} \int_{[-R, R]^{2}} p_{t-s}(x-y) p_{t-s}\left(x^{\prime}-y\right) d x d x^{\prime} d y \\
& =\int_{[-R, R]^{2}} p_{2(t-s)}\left(x-x^{\prime}\right) d x d x^{\prime} \\
& \leq 2 R .
\end{aligned}
$$

Then, using the isometry property of the stochastic integral we can compute the variance $\sigma_{R}^{2}$ :

$$
\begin{aligned}
\sigma_{R}^{2} & :=\int_{0}^{t} \int_{\mathbb{R}}\left(\int_{-R}^{R} p_{t-s}(x-y) d x\right)^{2} \mathbb{E}\left[\sigma(u(s, y))^{2}\right] d y d s \\
& =\int_{0}^{t} \mathcal{E}(s) \int_{\mathbb{R}} f_{R}^{2}(s, y) d y d s \\
& =\int_{0}^{t} \mathcal{E}(s) \int_{[-R, R]^{2}} p_{2(t-s)}\left(x-x^{\prime}\right) d x d x^{\prime} d s \\
& \approx 2 R \int_{0}^{t} \mathcal{E}(s) d s,
\end{aligned}
$$

as $R \rightarrow \infty$, with $\mathcal{E}(s)=\mathbb{E}\left[\sigma(u(s, y))^{2}\right]$. 
Fix $t>0$ and set

$$
F_{R}=\frac{1}{\sigma_{R}}\left(\int_{-R}^{R} u(t, x) d x-2 R\right) .
$$

The main result is the following quantitative version of the central limit theorem.

Theorem 4.2.1 (Huang, Nualart and Viitasaari [10]). Let $Z \sim$ $N(0,1)$. Then there exists a constant $C$, depending on $t$, such that

$$
d_{T V}\left(F_{R}, Z\right) \leq \frac{C}{\sqrt{R}}
$$

where $Z \sim N(0,1)$.

Proof. The main ingredient of the proof is the following result (see (2.7): If $F_{R} \in \mathbb{D}^{1,2}$ satisfies $\mathbb{E}\left[F_{R}^{2}\right]=1$ and $F_{R}=\delta\left(v_{R}\right)$, where $v_{R} \in \operatorname{Dom} \delta$. Then,

$$
d_{T V}\left(F_{R}, Z\right) \leq 2 \sqrt{\operatorname{Var}\left(\left\langle D F_{R}, v_{R}\right\rangle_{\mathfrak{H}}\right)}
$$

We can write

$$
F_{R}=\delta\left(v_{R}\right)
$$

where

$$
v_{R}(s, y)=\frac{1}{\sigma_{R}} \sigma(u(s, y)) f_{R}(s, y) \mathbf{1}_{[0, t]}(s)
$$

Moreover,

$$
D_{s, y} F_{R}=\frac{1}{\sigma_{R}}\left(\int_{-R}^{R} D_{s, y} u(t, x) d x\right) \mathbf{1}_{[0, t]}(s)
$$

Therefore,

$$
\left\langle D F_{R}, v_{R}\right\rangle_{\mathfrak{H}}=\frac{1}{\sigma_{R}^{2}} \int_{0}^{t} \int_{\mathbb{R}} \int_{[-R, R]} f_{R}(s, y) \sigma(u(s, y)) D_{s, y} u(t, x) d x d y d s .
$$

We know that $u(t, x) \in \mathbb{D}^{1, p}$ for all $p \geq 1$ and for $s \leq t$,

$$
\begin{aligned}
D_{s, y} u(t, x)= & p_{t-s}(x-y) \sigma(u(s, y)) \\
& +\int_{s}^{t} \int_{\mathbb{R}} p_{t-r}(x-z) \Sigma(r, z) D_{s, y} u(r, z) W(d r, d z),
\end{aligned}
$$

where $\Sigma(r, z)$ is an adapted random field bounded by the Lipschitz constant of $\sigma$. If $\sigma \in C^{1}$, then $\Sigma(r, z)=\sigma^{\prime}(u(r, z))$. This produces the decomposition

$$
\begin{aligned}
& \left\langle D F_{R}, v_{R}\right\rangle_{\mathfrak{H}} \\
= & \frac{1}{\sigma_{R}^{2}} \int_{0}^{t} \int_{\mathbb{R}} f_{R}^{2}(s, y) \sigma^{2}(u(s, y)) d y d s \\
& +\frac{1}{\sigma_{R}^{2}} \int_{0}^{t} \int_{\mathbb{R}} f_{R}(s, y) \sigma(u(s, y))\left(\int_{s}^{t} \int_{\mathbb{R}} f_{R}(r, z) \Sigma(r, z) D_{s, y} u(r, z) W(d r, d z)\right) d y d s .
\end{aligned}
$$


We have

$$
\sqrt{\operatorname{Var}\left(\left\langle D F_{R}, v_{R}\right\rangle_{\mathfrak{H}}\right)} \leq \sqrt{2}\left(A_{1}+A_{2}\right)
$$

where

$$
A_{1}=\frac{1}{\sigma_{R}^{2}} \int_{0}^{t}\left(\int_{\mathbb{R}^{2}} f_{R}^{2}(s, y) f_{R}^{2}\left(s, y^{\prime}\right) \operatorname{Cov}\left(\sigma^{2}(u(s, y)), \sigma^{2}\left(u\left(s, y^{\prime}\right)\right)\right) d y d y^{\prime}\right)^{\frac{1}{2}} d s
$$

and

$$
\begin{aligned}
A_{2}= & \frac{1}{\sigma_{R}^{2}} \int_{0}^{t}\left(\int_{\mathbb{R}^{2}} f_{R}(s, y) f_{R}\left(s, y^{\prime}\right) \int_{s}^{t} \int_{\mathbb{R}} f_{R}^{2}(r, z)\right. \\
& \left.\times \mathbb{E}\left[\sigma(u(s, y)) \sigma\left(u\left(s, y^{\prime}\right)\right) \Sigma^{2}(r, z) D_{s, y} u(r, z) D_{s, y^{\prime}} u(r, z)\right] d z d r d y d y^{\prime}\right)^{\frac{1}{2}} d s .
\end{aligned}
$$

Estimation of $A_{2}$ : Using

$$
\begin{gathered}
\sup _{y \in \mathbb{R}} \sup _{s \in[0, t]}\|\sigma(u(s, y))\|_{L^{4}(\Omega)} \leq K(t), \\
|\Sigma(r, z)| \leq \operatorname{Lip}(\sigma), \\
\left\|D_{s, y} u(r, z)\right\|_{L^{4}(\Omega)} \leq C p_{r-s}(z-y),
\end{gathered}
$$

and $f_{R}(s, y) f_{R}\left(s, y^{\prime}\right) \leq 1$ we obtain

$$
A_{2} \leq \frac{C}{R} \int_{0}^{t}\left(\int_{\mathbb{R}^{2}} \int_{s}^{t} \int_{\mathbb{R}} f_{R}^{2}(r, z) p_{r-s}(z-y) p_{r-s}\left(z-y^{\prime}\right) d z d r d y d y^{\prime}\right)^{\frac{1}{2}} d s .
$$

Integrating $y^{\prime}, y$ over $\mathbb{R}$, and using (4.3) we obtain

$$
A_{2} \leq \frac{C}{R} \int_{0}^{t}\left(\int_{s}^{t} \int_{\mathbb{R}^{2}} f_{R}^{2}(r, z) d z d r\right)^{\frac{1}{2}} d s \leq \frac{C}{\sqrt{R}} .
$$

Estimation of $A_{1}$ : $\quad$ By Clark-Ocone formula for two-parameter processes,

$$
\sigma^{2}(u(s, y))=\mathbb{E}\left[\sigma^{2}(u(s, y))\right]+\int_{0}^{s} \int_{\mathbb{R}} \mathbb{E}\left[D_{r, z}\left(\sigma^{2}(u(s, y))\right) \mid \mathcal{F}_{r}\right] W(d r, d z) .
$$

Then,

$$
\begin{aligned}
& \operatorname{Cov}\left(\sigma^{2}(u(s, y)), \sigma^{2}\left(u\left(s, y^{\prime}\right)\right)\right) \\
& \quad=\int_{0}^{s} \int_{\mathbb{R}} \mathbb{E}\left[\mathbb{E}\left[D_{r, z}\left(\sigma^{2}(u(s, y))\right) \mid \mathcal{F}_{r}\right] \mathbb{E}\left[D_{r, z}\left(\sigma^{2}\left(u\left(s, y^{\prime}\right)\right)\right) \mid \mathcal{F}_{r}\right]\right] d z d r .
\end{aligned}
$$

Applying the chain rule we have

$$
D_{r, z}\left(\sigma^{2}(u(s, y))\right)=2 \sigma(u(s, y)) \Sigma(s, y) D_{r, z} u(s, y)
$$


and

$$
\left\|\mathbb{E}\left[D_{r, z}\left(\sigma^{2}(u(s, y))\right) \mid \mathcal{F}_{r}\right]\right\|_{2} \leq 2 K_{4}(t) \operatorname{Lip}(\sigma)\left\|D_{r, z} u(s, y)\right\|_{4} .
$$

This produces the estimate

$$
\begin{aligned}
& \left|\operatorname{Cov}\left(\sigma^{2}(u(s, y)), \sigma^{2}\left(u\left(s, y^{\prime}\right)\right)\right)\right| \\
& \quad \leq 4 \operatorname{Lip}(\sigma)^{2} K_{4}^{2}(t) \int_{0}^{s} \int_{\mathbb{R}}\left\|D_{r, z} u(s, y)\right\|_{4}\left\|D_{r, z} u\left(s, y^{\prime}\right)\right\|_{4} d z d r \\
& \quad \leq C \int_{0}^{s} \int_{\mathbb{R}} p_{s-r}(z-y) p_{s-r}\left(z-y^{\prime}\right) d z d r \\
& \quad=C \int_{0}^{s} p_{2 s-2 r}\left(y-y^{\prime}\right) d r .
\end{aligned}
$$

Therefore,

$$
\begin{aligned}
A_{1} & \leq \frac{C}{R} \int_{0}^{t}\left(\int_{0}^{s} \int_{\mathbb{R}^{2}} f_{R}^{2}(s, y) f_{R}^{2}\left(s, y^{\prime}\right) p_{2 s-2 r}\left(y-y^{\prime}\right) d r d y d y^{\prime}\right)^{\frac{1}{2}} d s \\
& \leq \frac{C}{R} \int_{0}^{t}\left(\int_{0}^{s} \int_{\mathbb{R}^{2}} f_{R}(s, y) f_{R}\left(s, y^{\prime}\right) p_{2 s-2 r}\left(y-y^{\prime}\right) d r d y d y^{\prime}\right)^{\frac{1}{2}} d s .
\end{aligned}
$$

We have, by the semigroup property, integrating in $y$ and $y^{\prime}$,

$\int_{\mathbb{R}^{2}} f_{R}(s, y) f_{R}\left(s, y^{\prime}\right) p_{2 s-2 r}\left(y-y^{\prime}\right) d y d y^{\prime}=\int_{[-R, R]^{2}} p_{2 t-2 r}\left(x-x^{\prime}\right) d x d x^{\prime} \leq 2 R$, which yields,

$$
A_{1} \leq \frac{C}{\sqrt{R}}
$$

This completes the proof.

\subsubsection{Functional Central Limit Theorem}

The following result is a functional version of the central limit theorem for space averages.

Theorem 4.2.2 (Huang, Nualart and Viitasaari [10]). Set $\xi(s)=$ $\mathbb{E}\left[\sigma(u(s, y))^{2}\right]$ for any $s \geq 0$. Then

$$
\left(\frac{1}{\sqrt{R}}\left(\int_{-R}^{R} u(t, x) d x-2 R\right)\right)_{t \in[0, T]} \rightarrow\left(\int_{0}^{t} \sqrt{2 \xi(s)} d B_{s}\right)_{t \in[0, T]}
$$

as $R$ tends to infinity, where $B$ is a Brownian motion and the convergence is in law on the space of continuous functions $C([0, T])$. 
Proof. The proof will be done in two steps.

(i) Proof of tightness: Tightness is a consequence of the estimate:

$$
\mathbb{E}\left(\left|\int_{-R}^{R} u(t, x) d x-\int_{-R}^{R} u(s, x) d x\right|^{p}\right) \leq C(T) R^{\frac{p}{2}}(t-s)^{\frac{p}{2}} .
$$

for any $0 \leq s<t \leq T$ and any $p \geq 1$.

(ii) Convergence of the finite-dimensional distributions: We use the following result:

Proposition 4.2.1. Let $F=\left(F^{(1)}, \ldots, F^{(m)}\right)$ be a random vector such that $F^{(i)}=\delta\left(v^{(i)}\right)$ for $v^{(i)} \in \operatorname{Dom} \delta, i=1, \ldots, m$. Assume $F^{(i)} \in \mathbb{D}^{1,2}$ for $i=1, \ldots, m$. Let $Z$ be an $m$-dimensional Gaussian centered vector with covariance matrix $\left(C_{i, j}\right)_{1 \leq i, j \leq m}$. For any $C^{2}$ function $h: \mathbb{R}^{m} \rightarrow \mathbb{R}$ with bounded second partial derivatives, we have

$$
\left|\mathbb{E}\left(h\left(F_{R}\right)\right)-\mathbb{E}(h(Z))\right| \leq \frac{m}{2}\left\|h^{\prime \prime}\right\|_{\infty} \sqrt{\sum_{i, j=1}^{m} \mathbb{E}\left[\left(C_{i, j}-\left\langle D F^{(i)}, v^{(j)}\right\rangle_{\mathfrak{H}}\right)^{2}\right]},
$$

where

$$
\left\|h^{\prime \prime}\right\|_{\infty}=\max _{1 \leq i, j \leq m} \sup _{x \in \mathbb{R}^{m}}\left|\frac{\partial^{2} h}{\partial x_{i} \partial x_{j}}(x)\right| .
$$

Fix points $0 \leq t_{1}<\cdots<t_{m} \leq T$ and consider the random variables

$$
F_{R}^{(i)}=\frac{1}{\sqrt{R}}\left(\int_{-R}^{R} u\left(t_{i}, x\right) d x-2 R\right),
$$

for $i=1, \ldots, m$. We can write $F_{R}^{(i)}=\delta\left(v_{R}^{(i)}\right)$, where

$$
v_{R}^{(i)}(s, y)=\mathbf{1}_{\left[0, t_{i}\right]}(s) \frac{1}{\sqrt{R}} \int_{-R}^{R} p_{t_{i}-s}(x-y) \sigma(u(s, y)) d x .
$$

Set $F_{R}=\left(F_{R}^{(1)}, \ldots, F_{R}^{(m)}\right)$ and let $Z$ be an $m$-dimensional Gaussian centered vector with covariance

$$
C_{i, j}:=\mathbb{E}\left[Z^{i} Z^{j}\right]=2 \int_{0}^{t_{i} \wedge t_{j}} \xi(r) d r,
$$

where we recall that $\xi(r)=\mathbb{E}\left[\sigma(2(r, x))^{2}\right]$.

Then, applying the previous proposition,

$$
\left|\mathbb{E}\left(h\left(F_{R}\right)\right)-\mathbb{E}(h(Z))\right| \leq \frac{m}{2}\left\|h^{\prime \prime}\right\|_{\infty} \sqrt{\sum_{i, j=1}^{m} \mathbb{E}\left[\left(C_{i, j}-\left\langle D F_{R}^{(i)}, v_{R}^{(j)}\right\rangle_{\mathfrak{H}}\right)^{2}\right]} .
$$


It suffices to show that for each $i, j,\left\langle D F_{R}^{(i)}, v_{R}^{(j)}\right\rangle_{\mathfrak{H}}$ converges in $L^{2}(\Omega)$, as $R$ tends to infinity to $C_{i, j}$. This follows from the expression

$$
\begin{aligned}
\left\langle D F_{R}^{(i)}, v_{R}^{(j)}\right\rangle_{\mathfrak{H}}=\frac{1}{R} & \int_{0}^{t_{i} \wedge t_{j}} \int_{\mathbb{R}} f_{R}\left(t_{i}, s, y\right) f_{R}\left(t_{j}, s, y\right) \sigma^{2}(u(s, y)) d y d s \\
& +\frac{1}{R} \int_{0}^{t_{i} \wedge t_{j}} \int_{\mathbb{R}} f_{R}\left(t_{j}, s, y\right) \sigma(u(s, y)) \\
& \times\left(\int_{s}^{t_{i}} \int_{\mathbb{R}} f_{r}\left(t_{i}, r, z\right) \Sigma(r, z) D_{s, y} u(r, z) W(d r, d z)\right) d y d s
\end{aligned}
$$

with the notation $f_{R}(t, s, y)=\int_{-R}^{R} p_{t-s}(x, y) d x$.

\subsubsection{Spatial colored noise}

Consider the stochastic heat equation

$$
\frac{\partial u}{\partial t}=\frac{1}{2} \Delta u+\sigma(u) \dot{W},
$$

on $\mathbb{R}_{+} \times \mathbb{R}^{d}$ with initial condition $u(0, x)=1$. The function $\sigma$ is Lipschitz continuous and we assume that $\sigma(1) \neq 0$.

The noise $\dot{W}(t, x)$ is a centered Gaussian random field with covariance

$$
\mathbb{E}[\dot{W}(t, x) \dot{W}(s, y)]=\delta_{0}(t-s) \gamma(x-y),
$$

where $\gamma: \mathbb{R}^{d} \rightarrow \mathbb{R}_{+} \cup\{\infty\}$ is nonnegative definite and the Fourier transform of $\gamma$ is a tempered measure $\mu$, that satisfies Dalang's condition:

$$
\int_{\mathbb{R}^{d}} \frac{\mu(d \xi)}{1+|\xi|^{2}}<\infty
$$

We define formally the noise as a Gaussian centered family of random variables

$$
W=\left\{W(\varphi), \varphi \in C_{0}^{\infty}\left([0, \infty) \times \mathbb{R}^{d}\right)\right\},
$$

with covariance

$$
\begin{aligned}
\mathbb{E}[W(\phi) W(\varphi)] & =\int_{0}^{\infty} \int_{\mathbb{R}^{2 d}} \phi(s, x) \varphi(s, y) \gamma(x-y) d x d y d s \\
& =\int_{0}^{\infty} \int_{\mathbb{R}^{d}} \mathcal{F} \phi(s, \xi) \overline{\mathcal{F} \varphi(s, \xi)} \mu(d \xi) d s,
\end{aligned}
$$

where $\mathcal{F} \phi$ refers to the Fourier transform in the space variable. Let $\mathfrak{H}_{0}$ be the closure of $C_{0}^{\infty}\left(\mathbb{R}^{d}\right)$ under the inner product

$$
\langle\varphi, \psi\rangle_{\mathfrak{H}_{0}}=\int_{\mathbb{R}^{2 d}} \varphi(x) \psi(y) \gamma(x-y) d x d y=\int_{\mathbb{R}^{d}} \mathcal{F} \varphi(\xi) \overline{\mathcal{F} \psi(\xi)} \mu(d \xi) .
$$


Then, the Gaussian family $W$ can be extended to the Hilbert space $\mathfrak{H}:=L^{2}\left(\mathbb{R}_{+}, \mathfrak{H}_{0}\right)$, in such a way that $\left\{W(g), g \in L^{2}\left(\mathbb{R}_{+} ; \mathfrak{H}_{0}\right)\right\}$ is an isonormal Gaussian process.

From Dalang [7] there is a unique mild solution, which is an adapted random field $u$ such that for all $p \geq 2$,

$$
\sup _{x \in \mathbb{R}} \sup _{0 \leq t \leq T} \mathbb{E}\left[|u(t, x)|^{p}\right]<\infty
$$

and $u$ satisfies the integral equation:

$$
u(t, x)=1+\int_{0}^{t} \int_{\mathbb{R}} p_{t-s}(x-y) \sigma(u(s, y)) W(d s, d y)
$$

where $p_{t}(x)=(2 \pi t)^{-d / 2} e^{-|x|^{2} / 2 t}$.

Consider the spacial averages

$$
F_{R}=\int_{B_{R}} u(t, x) d x
$$

where $B_{R}=\left\{x \in \mathbb{R}^{d}:|x| \leq R\right\}$. Chen, Khoshnevisan, Nualart and $\mathrm{Pu}$ (work in progress) proved that if $\int_{\mathbb{R}^{d}} \gamma(x) d x<\infty$, then, for all $t>0$,

$$
d_{T V}\left(\frac{1}{\sigma_{R}} \int_{B_{R}}[u(t, x)-1] d x, Z\right) \leq C R^{-d / 2},
$$

where $\sigma_{R}^{2}=\operatorname{Var}\left(\int_{B_{R}}[u(t, x)-1] d x\right)$. Moreover, as $R \rightarrow+\infty$,

$$
\sigma_{R}^{2} \sim R^{d} \int_{\mathbb{R}^{d}} \operatorname{Cov}(u(t, x), u(t, 0)) d x .
$$

For the Riesz kernel $\gamma(x)=|x|^{-\beta}$, with $0<\beta<\min (d, 2)$, that is not integrable, we have the following result.

Theorem 4.2.3 (Huang, Nualart, Viitasaari and Zheng [11]). For all $t>0$, there exists a constant $C=C(t, \beta)$, such that

$$
d_{T V}\left(\frac{1}{\sigma_{R}} \int_{B_{R}}[u(t, x)-1] d x, Z\right) \leq C R^{-\beta / 2}
$$

where $\sigma_{R}^{2}=\operatorname{Var}\left(\int_{B_{R}}[u(t, x)-1] d x\right)$. Moreover, as $R \rightarrow+\infty$,

$$
\sigma_{R}^{2} \sim\left(k_{\beta} \int_{0}^{t} \eta^{2}(s) d s\right) R^{2 d-\beta}
$$

where $\eta(s)=\mathbb{E}[\sigma(u(s, y))]$ and $k_{\beta}:=\int_{B_{1}^{2}}\left|x_{1}-x_{2}\right|^{-\beta} d x_{1} d x_{2}$. 
In [11]], the authors have also obtained a functional version of the above central limit theorem. That means, as $R \rightarrow+\infty$, we have

$$
\left(R^{\frac{\beta}{2}-d} \int_{B_{R}}[u(t, x)-1] d x\right)_{t \in[0, T]} \Rightarrow\left(\sqrt{k_{\beta}} \int_{0}^{t} \eta(s) d B_{s}\right)_{t \in[0, T]},
$$

where $B$ is a Brownian motion and the convergence takes place on the space of continuous functions $C([0, T])$. If $\sigma(x)=x$, then the first chaos dominates (non-chaotic behavior). This is not true for space-time white noise and $\sigma(x)=x$.

\subsubsection{Stochastic wave equation}

Consider the stochastic wave equation on on $\mathbb{R}_{+} \times \mathbb{R}$

$$
\frac{\partial^{2} u}{\partial t^{2}}=\frac{\partial^{2} u}{\partial x^{2}}+\sigma(u) \dot{W}
$$

where $\dot{W}=\frac{\partial^{2} W}{\partial t \partial x}$ and $W$ is a two parameter centered Gaussian process with covariance

$$
\mathbb{E}[W(t, x) W(s, y)]=(s \wedge t) \frac{1}{2}\left(|x|^{2 H}+|y|^{2 H}-|x-y|^{2 H}\right),
$$

where $H \in[1 / 2,1)$. That is, $W$ is a Brownian motion in time and a fractional Brownian motion with Hurst parameter $H$ is space.

We assume the initial conditions $u(0, x)=1$ and $\frac{\partial u}{\partial x} u(0, x)=0$ and $\sigma$ is Lipschtiz. There is a unique mild solution such that

$$
u(t, x)=1+\frac{1}{2} \int_{0}^{t} \int_{\mathbb{R}} \mathbf{1}_{\{|x-y| \leq t-s\}} \sigma(u(s, y)) W(d s, d y) .
$$

Set

$$
F_{R}(t):=\frac{1}{\sigma_{R}}\left(\int_{-R}^{R} u(t, x) d x-2 R\right),
$$

where $\sigma_{R}^{2}=\operatorname{Var}\left(\int_{-R}^{R} u(t, x) d x\right)$. Then, we can prove the following version of the central limit theorem for spatial averages.

Theorem 4.2.4 (Delgado, Nualart and Zheng [8]). Let $Z \sim N(0,1)$. Then there exists a constant $C$, depending on $t$, such that

$$
d_{T V}\left(F_{R}(t), Z\right) \leq C R^{H-1} .
$$

It is also possible to show the following functional central limit theorem. Set $\eta(s)=\mathbb{E}[\sigma(u(s, y))]$ and $\mathcal{E}(s)=\mathbb{E}\left[\sigma^{2}(u(s, y))\right], s \geq 0$. 
Theorem 4.2.5 (Delgado-Nualart-Zheng [8]). As $R$ tends to infinity,

(i) If $H=\frac{1}{2}$,

$\left.\left(\frac{1}{\sqrt{R}}\left(\int_{-R}^{R} u(t, x) d x-2 R\right)\right)_{t \in[0, T]} \rightarrow\left(\int_{0}^{t}(t-s) \sqrt{2 \mathcal{E}(s)}\right) d B_{s}\right)_{t \in[0, T]}$,

(ii) If $H \in(1 / 2,1)$,

$\left(R^{-H}\left(\int_{-R}^{R} u(t, x) d x-2 R\right)\right)_{t \in[0, T]} \rightarrow\left(\sqrt{2} \int_{0}^{t}(t-s) \eta(s) d B_{s}\right)_{t \in[0, T]}$,

where $B$ is a Brownian motion and the convergence is in law in $C([0, T])$.

We would like to point out that in (ii) the first chaos dominates when $\sigma(x)=x$. 


\section{Bibliography}

[1] S. Ben Hariz. Limit theorems for the non-linear functionals of stationary Gaussian processes. J. Mult. Anal. 80, pp. 191-216, (2002).

[2] H. Biermé, A. Bonami, I. Nourdin and G. Peccati. Optimal BerryEsseen rates on the Wiener space: the barrier of third and fourth cumulants. ALEA 9, no 2., pp. 473-500, (2012).

[3] J. M. Bismut. Martingales, the Malliavin calculus and hypoellipticity under general Hörmander's condition. Z. Wahrscheinlichkeitstheorie verw. Gebiete 63, pp. 147-235, (1981).

[4] P. Breuer and P. Major. Central limit theorems for non-linear functionals of Gaussian fields. J. Mult. Anal. 13, pp. 425-441, (1983).

[5] D. Chambers and E. Slud. Central limit theorems for nonlinear functionals of stationary Gaussian processes. Probab. Th. Rel. Fields 80, pp. 323-346, (1989).

[6] L. H. Y. Chen, L. Goldstein and Qi-Man Shao. Normal approximation by Stein's method. Springer, 2011.

[7] R. Dalang. Extending martingale measure stochastic integral with applications to spatially homogeneous S. P. D. E's. Electron. Journal Probab. 4, no. 6, 29 pp., (1999).

[8] F. Delgado, D. Nualart and G. Zheng. A Central Limit Theorem for the stochastic wave equation with fractional noise. Preprint.

[9] Y. Hu and D. Nualart. Renormalized self-intersection local time for fractional Brownian motion. The Annals of Probability 33, pp. 948983, (2005).

[10] J. Huang, D. Nualart and L. Viitasaari. A Central Limit Theorem for the stochastic heat equation. Preprint. 
[11] J. Huang, D. Nualart, L. Viitasaari and G. Zheng. Gaussian fluctuations for the stochastic heat equation with colored noise. Preprint.

[12] S. Kusuola and D. W. Stroock. Applications of the Malliavin calculus I. In: Stochastic Analysis, Proc. Taniguchi Inter. Symp. on Stochastic Analysis, Katata and Kyoto 1982, ed. K. Itô, Kinokuniya/NorthHolland, Tokyo, pp. 271-306, (1984).

[13] P. Malliavin. Stochastic calculus of variation and hypoelliptic operators, In: Proceedings of the International Symposium on Stochastic Differential Equations (Res. Inst. Math. Sci., Kyoto Univ., Kyoto, 1976), Wiley, New York-Chichester-Brisbane, pp. 195-263, (1978).

[14] I. Nourdin and D. Nualart. The functional Breuer-Major theorem. Probab. Theory and Rel. Fields. To appear.

[15] I. Nourdin, D. Nualart and Peccati. The Breuer-Major Theorem in total variation: improved rates under minimal regularity. In preparation.

[16] I. Nourdin and G. Peccati. Normal approximations with Malliavin calculus: from Stein's method to universality. Cambridge tracts in Mathematics 192, Cambridge University Press, 2012.

[17] I. Nourdin and G. Peccati. The optimal fourth moment theorem. Proc. Amer. Math. Soc. 143, no. 7, pp. 3123-3133, (2015).

[18] I. Nourdin, G. Peccati and X. Yang. Berry-Esseen bounds in the Breuer-Major CLT and Gebelein's inequality. Electron. Commun. Probab. 24, no. 34, 12 pp., (2019).

[19] D. Nualart. The Malliavin calculus and related topics. 2nd edition. Probability and Its Applications, Springer, 2006.

[20] D. Nualart and E. Nualart. Introduction to Malliavin Calculus. Institute of Mathematical Statistics Textbooks, Cambridge University Press, 2018.

[21] D. Nualart and E. Pardoux. Stochastic calculus with anticipating integrands. Probab. Theory Rel. Fields 78 pp. 535-581, (1988).

[22] D. Nualart and G. Peccati. Central limit theorems for sequences of multiple stochastic integrals. The Annals of Probability 33, pp. 177193, (2005).

[23] D. Nualart and S. Ortiz-Latorre. Central limit theorems for multiple stochastic integrals. Stochastic Proc. Appl. 118, pp. 614-628, (2007). 
[24] D. Nualart and H. Zhou. Total variation estimates in the BreuerMajor theorem. Preprint.

[25] G. Peccati and C. Tudor. Gaussian limits for vector-valued multiple stochastic integrals . Séminaire de Probabilités XXXVIII, pag 247-262. Lecture Notes in Math., 1857, Springer Verlag, Berlin, 2005.

[26] G. Pisier. Riesz transforms. A simple analytic proof of P. A. Meyer's inequality. Lecture Note in Mathematics 1321, pp. 485-501, (1988).

[27] A. V. Skorohod. On a generalization of a stochastic integral. Theory Probab. Appl. 20, pp. 219-233, (1975).

[28] D. W. Stroock. Some applications of stochastic calculus to partial differential equations. In: Ecole d'Eté de Probabilités de Saint Flour, Lecture Notes in Math. 976, pp. 267-382, (1983).

[29] J. B. Walsh. An introduction to stochastic partial differential equations. In: Ecole d'Eté de Probabilités de Saint Flour XIV, Lecture Notes in Math. 1180, pp. 265-438, (1986).

[30] S. Watanabe. Lectures on Stochastic Differential Equations and Malliavin Calculus. Tata Institute of Fundamental Research, Springer-Verlag, 1984.

David Nualart

Department of Mathematics

The University of Kansas

Lawrence, Kansas, 66045, USA

http://nualart.faculty.ku.edu/ 